\title{
MONOIDAL CATEGORIES ASSOCIATED WITH STRATA OF FLAG MANIFOLDS
}

\author{
MASAKI KASHIWARA, MYUNGHO KIM, SE-JIN OH, AND EUIYONG PARK
}

\begin{abstract}
We construct a monoidal category $\mathscr{C}_{w, v}$ which categorifies the doublyinvariant algebra ${ }^{N^{\prime}(w)} \mathbb{C}[N]^{N(v)}$ associated with Weyl group elements $w$ and $v$. It gives, after a localization, the coordinate algebra $\mathbb{C}\left[\mathcal{R}_{w, v}\right]$ of the open Richardson variety associated with $w$ and $v$. The category $\mathscr{C}_{w, v}$ is realized as a subcategory of the graded module category of a quiver Hecke algebra $R$. When $v=$ id, $\mathscr{C}_{w, v}$ is the same as the monoidal category which provides a monoidal categorification of the quantum unipotent coordinate algebra $A_{q}(\mathfrak{n}(w))_{\mathbb{Z}\left[q, q^{-1}\right]}$ given by Kang-KashiwaraKim-Oh. We show that the category $\mathscr{C}_{w, v}$ contains special determinantial modules $\mathrm{M}\left(w_{\leq k} \Lambda, v_{\leq k} \Lambda\right)$ for $k=1, \ldots, \ell(w)$, which commute with each other. When the quiver Hecke algebra $R$ is symmetric, we find a formula of the degree of $R$-matrices between the determinantial modules $\mathrm{M}\left(w_{\leq k} \Lambda, v_{\leq k} \Lambda\right)$. When it is of finite $A D E$ type, we further prove that there is an equivalence of categories between $\mathscr{C}_{w, v}$ and $\mathscr{C}_{u}$ for $w, u, v \in \mathrm{W}$ with $w=v u$ and $\ell(w)=\ell(v)+\ell(u)$.
\end{abstract}

\section{Contents}

Introduction 2

1. Preliminaries 5

1.1. Quantum groups 5

1.2. Quiver Hecke algebras 9

$\begin{array}{ll}\text { 1.3. Convex preorders } & 13\end{array}$

2010 Mathematics Subject Classification. 16D90, 18D10, 81R10.

Key words and phrases. Categorification, Monoidal category, Quantum cluster algebra, Quiver Hecke algebra, Richardson variety.

The research of M.Ka. was supported by Grant-in-Aid for Scientific Research (B) 15H03608, Japan Society for the Promotion of Science.

The research of M. Kim was supported by the National Research Foundation of Korea(NRF) Grant funded by the Korea government(MSIP) (NRF-2017R1C1B2007824).

The research of S.-j. Oh was supported by the National Research Foundation of Korea(NRF) Grant funded by the Korea government(MSIP) (NRF-2016R1C1B2013135).

The research of E.P. was supported by the National Research Foundation of Korea(NRF) Grant funded by the Korean Government(MSIP)(NRF-2017R1A1A1A05001058). 
2. Categorification of Doubly-invariant algebras 19

2.1. Cuspidal decomposition 19

2.2. Categories $\mathscr{C}_{w, v} \quad 26$

3. R-matrices 32

3.1. Strongly commuting pairs 32

3.2. Normalized R-matrices 33

4. Determinantial modules 36

5. Finite ADE types 44

References $\quad 48$

\section{INTRODUCTION}

A quantum cluster algebra is a non-commutative q-deformation of a cluster algebra which is a special $\mathbb{Z}$-subalgebra of a rational function field with a set of generators grouped into overlapping subsets, called clusters, and a procedure which makes new clusters, called mutation. The (quantum) cluster algebras appear naturally in various studies of mathematics including representation theory of Kac-Moody algebras. Let $\mathfrak{g}$ be a Kac-Moody algebra of symmetric type and $U_{q}(\mathfrak{g})$ its quantum group. We take an element $w$ of the Weyl group W. It was shown in [3] that the quantum unipotent coordinate algebra $A_{q}(\mathfrak{n}(w))$ associated with $U_{q}(\mathfrak{g})$ and $w$ has a quantum cluster algebra structure. It turned out that the intersection of the upper global basis and $A_{q}(\mathfrak{n}(w))$ is a basis of $A_{q}(\mathfrak{n}(w))$ ([15]). On the other hand, it was proved that there is a cluster algebra structure inside the coordinate ring of a Richardson variety. For $w, v \in \mathrm{W}$ with $v \leq w$, one can consider the open Richardson variety $\mathcal{R}_{w, v}$ which is the intersection of the Schubert cell and the opposite Schubert cell attached to $w$ and $v$ respectively. It was shown in [17] that, when $\mathfrak{g}$ is of finite $A D E$ type, the coordinate algebra $\mathbb{C}\left[\mathcal{R}_{w, v}\right]$ contains a subalgebra with the cluster algebra structure coming from a certain subcategory of module category of a preprojective algebra which is determined by $w, v$. The cluster variables of the initial cluster are given by the irreducible factors of the generalized minors determined by $w_{\leq j}$ and $v_{\leq j}$ for $j \in J_{\underline{w}, v}$ (for these notations, see (5.5)). A quantization of the coordinate algebra $\mathbb{C}\left[\mathcal{R}_{w, v}\right]$ was studied in [18] in the aspect of quantum cluster algebras.

The quiver Hecke algebras (or Khovanov-Lauda-Rouquier algebras) were introduced to categorify the half of a quantum group $([13,14,21])$. The algebras have special quotients, called cyclotomic quiver Hecke algebras, which provide a categorification of irreducible integrable highest weight modules ([4]). It was shown in $[1,21]$ that 
cyclotomic quiver Hecke algebras of affine type $A$ are isomorphic to cyclotomic Hecke algebras. In that sense, the quiver Hecke algebras are a vast generalization of Hecke algebra in the direction of categorification. When the quiver Hecke algebra is symmetric and the base field is of characteristic zero, the upper global basis corresponds to the set of isomorphism classes of simple modules $([22,25])$.

Let $R(\beta)$ be the symmetric quiver Hecke algebra corresponding to a quantum group $U_{q}(\mathfrak{g})$ of symmetric type and denote by $R$-mod the category of graded $R$-modules which are finite-dimensional over the base field $\mathbf{k}$. It was proved in [7] that there is a monoidal subcategory $\mathcal{C}_{w}$ of $R$-mod which provides a monoidal categorification of $A_{q}(\mathfrak{n}(w))$ as a quantum cluster algebra. The determinantial modules $\mathrm{M}(\lambda, \mu)$ are a key ingredient for the monoidal categorification. They are simple $R$-modules determined by a dominant integral weight $\Lambda$ and $\lambda, \mu \in \mathrm{W} \Lambda$ with $\lambda \leq \mu$, which correspond to the unipotent quantum minors under the categorification. The determinantial modules defined by a reduced expression $\underline{w}$ and $R$-matrices among them give a quantum monoidal seed in $\mathcal{C}_{w}$. This monoidal categorification gave a proof of the conjecture that any cluster monomial is a member of the upper global basis up to a power of $q^{1 / 2}$.

In this paper, we construct a monoidal category $\mathscr{C}_{w, v}$ associated with Weyl group elements $w$ and $v$ as a subcategory of $R$-mod for an arbitrary quiver Hecke algebra. The results on convex orders and cuspidal modules obtained in [24] play a key role in proving properties on $\mathscr{C}_{w, v}$. Let $\Delta_{+}$(resp. $\Delta_{-}$) be the set of positive roots (resp. negative roots) and set $\mathrm{Q}_{+}:=\sum_{i \in I} \mathbb{Z}_{\geq 0} \alpha_{i}$ and $\mathrm{Q}_{-}:=-\mathrm{Q}_{+}$. For an $R(\beta)$-module $M$, we define

$$
\begin{aligned}
\mathbf{W}(M) & :=\left\{\gamma \in \mathrm{Q}_{+} \cap\left(\beta-\mathrm{Q}_{+}\right) \mid e(\gamma, \beta-\gamma) M \neq 0\right\}, \\
\mathbf{W}^{*}(M) & :=\left\{\gamma \in \mathrm{Q}_{+} \cap\left(\beta-\mathrm{Q}_{+}\right) \mid e(\beta-\gamma, \gamma) M \neq 0\right\} .
\end{aligned}
$$

For $w, v \in \mathrm{W}$, let us denote by $\mathscr{C}_{w, v}$ the full subcategory of $R$-mod whose objects $M$ satisfy

$$
\mathrm{W}(M) \subset \mathrm{Q}_{+} \cap w \mathrm{Q}_{-} \quad \text { and } \quad \mathrm{W}^{*}(M) \subset \mathrm{Q}_{+} \cap v \mathrm{Q}_{+} .
$$

The category $\mathscr{C}_{w, v}$ is stable under taking subquotients, extensions, convolution products and grading shifts (Proposition 2.16). When $v=\mathrm{id}, \mathscr{C}_{w, v}$ is nothing but the monoidal category $\mathcal{C}_{w^{-1}}$ introduced in [7]. We also give a membership condition on $\mathscr{C}_{w, v}$ using the cuspidal decomposition in Proposition 2.18. For $w, v \in \mathbf{W}$, we define $A_{w, v}$ to be the $\mathbb{Z}\left[q, q^{-1}\right]$-linear subspace of $A_{q}(\mathfrak{n})_{\mathbb{Z}\left[q, q^{-1}\right]}$ consisting of all elements $x \in A_{q}(\mathfrak{n})_{\mathbb{Z}\left[q, q^{-1}\right]}$ such that

$$
e_{i_{1}} \cdots e_{i_{l}} x=0 \quad \text { and } \quad e_{j_{1}}^{*} \cdots e_{j_{l^{\prime}}}^{*} x=0
$$


for any $\left(i_{1}, \ldots, i_{l}\right) \in I^{\beta}$ with $\beta \in Q_{+} \cap w Q_{+} \backslash\{0\}$ and any $\left(j_{1}, \ldots, j_{l^{\prime}}\right) \in I^{\gamma}$ with $\gamma \in$ $\mathrm{Q}_{+} \cap v \mathrm{Q}_{-} \backslash\{0\}$. Theorem 2.20 tells that the Grothendieck ring of $\mathscr{C}_{w, v}$ is equal to $A_{w, v}$ under the categorification, which implies that $A_{w, v}$ forms a subalgebra of $A_{q}(\mathfrak{n})_{\mathbb{Z}\left[q, q^{-1}\right]}$. The subalgebra $A_{w, v}$ can be understood as a $q$-deformation of the doubly-invariant algebra ${ }^{N^{\prime}(w)} \mathbb{C}[N]^{N(v)}$, where $N$ is the unipotent radical, $N^{\prime}(w)=N \cap\left(w N w^{-1}\right)$ and $N(v)=N \cap\left(v N^{-} v^{-1}\right)$ (Remark 2.19).

The category $\mathscr{C}_{w, v}$ contains special determinantial modules related to $w$ and $v$. Fix a reduced expression $\underline{w}$ of $w$ and define $w_{\leq k}$ and $v_{\leq k}$ by (4.2) and (4.3) for $k=1, \ldots, \ell(w)$. It is proved in Proposition 4.8 that the determinantial modules $\mathrm{M}\left(w_{\leq k} \Lambda, v_{\leq k} \Lambda\right)$ are contained in $\mathscr{C}_{w, v}$ for any dominant integral weight $\Lambda$. We then show that the determinantial modules $\mathrm{M}\left(w_{\leq k} \Lambda, v_{\leq k} \Lambda\right)$ commute with each other (Theorem 4.10). When the quiver Hecke algebra $R$ is symmetric, we compute the degree of $R$-matrices between them in Theorem 4.12 as follows:

$$
\Lambda\left(\mathrm{M}\left(w_{\leq j} \Lambda, v_{\leq j} \Lambda\right), \mathrm{M}\left(w_{\leq k} \Lambda^{\prime}, v_{\leq k} \Lambda^{\prime}\right)\right)=\left(w_{\leq j} \Lambda+v_{\leq j} \Lambda, v_{\leq k} \Lambda^{\prime}-w_{\leq k} \Lambda^{\prime}\right)
$$

for $1 \leq k<j \leq \ell$. When specialized at $q=1$, the determinantial modules $\mathrm{M}\left(w_{\leq k} \Lambda, v_{\leq k} \Lambda\right)$ correspond to the generalized minors associated with $w_{\leq k}$ and $v_{\leq k}$ which provide the initial cluster in the coordinate ring $\mathbb{C}\left[\mathcal{R}_{w, v}\right]$ of the open Richardson variety in [17]. Note that the coordinate ring $\mathbb{C}\left[\mathcal{R}_{w, v}\right]$ is a localization of the doublyinvariant algebra ${ }^{\prime}(w) \mathbb{C}[N]^{N(v)}$ by a subset of such generalized minors ([17, Theorem 2.12]). It is expected that our category $\mathscr{C}_{w, v}$ gives a monoidal categorification of a quantization of the cluster algebra arising from $\mathcal{R}_{w, v}$ given in [17].

Suppose that $\mathfrak{g}$ is of finite $A D E$ type. Then S. Kato ([11]) constructed the Saito reflection functors

$$
\mathcal{T}_{i}: R_{i}(\beta)-\bmod \stackrel{\sim}{\longrightarrow}{ }_{i} R\left(s_{i} \beta\right)-\bmod , \quad \mathcal{T}_{i}^{*}:{ }_{i} R(\beta)-\bmod \stackrel{\sim}{\longrightarrow} R_{i}\left(s_{i} \beta\right)-\bmod ,
$$

which are equivalences of categories (see $\S 5$ ). We show that they induce equivalences of categories (Theorem 5.3):

$$
\left.\mathcal{T}_{i}\right|_{\mathscr{C}_{s_{i} w, s_{i} v}}: \mathscr{C}_{s_{i} w, s_{i} v} \stackrel{\sim}{\longrightarrow} \mathscr{C}_{w, v},\left.\quad \mathcal{T}_{i}^{*}\right|_{\mathscr{C}_{w, v}}: \mathscr{C}_{w, v} \stackrel{\sim}{\longrightarrow} \mathscr{C}_{s_{i} w, s_{i} v}
$$

for $w, v \in \mathrm{W}$ such that $s_{i} w>w$ and $s_{i} v>v$, Thus, when $w, u, v \in \mathrm{W}$ such that $w=v u$ and $\ell(w)=\ell(v)+\ell(u)$, there is a category equivalence between $\mathscr{C}_{w, v}$ and $\mathscr{C}_{u}$. After finishing the paper, it was proved by S. Kato ([12]) and P. J. McNamara ([19]) independently that the reflection functors are monoidal, which implies that Conjecture 5.5 is true (Remark 5.6).

The paper is organized as follows. In Section 1, we review quantum groups, quiver Hecke algebras and results on the convex preorders given in [24]. We also prove several 
lemmas on properties of convex preorders. In Section 2, we construct and investigate the categories $\mathscr{C}_{w, v}$ and the algebras $A_{w, v}$, and prove the main theorem for $\mathscr{C}_{w, v}$ and $A_{w, v}$. Section 3 contains a review on $R$-matrices and new lemmas for proving the formula of the degree of $R$-matrices between the determinantial modules. In Section 4 , we show the commuting property on $\mathrm{M}\left(w_{\leq k} \Lambda, v_{\leq k} \Lambda\right)$ and give a formula to compute the degree $\Lambda\left(\mathrm{M}(w \Lambda, v \Lambda), \mathrm{M}\left(w_{\leq k} \Lambda^{\prime}, v_{\leq k} \Lambda^{\prime}\right)\right)$ of $R$-matrices when the quiver Hecke algebra is symmetric. Section 5 specializes to the case of finite $A D E$ types. We show that there is a category equivalence between $\mathscr{C}_{s_{i} w, s_{i} v}$ and $\mathscr{C}_{w, v}$ for $w, v \in \mathrm{W}$ with $s_{i} w>w$ and $s_{i} v>v$, which yields a category equivalence between between $\mathscr{C}_{w, v}$ and $\mathscr{C}_{u}$ when $w, u, v \in \mathrm{W}$ such that $w=v u$ and $\ell(w)=\ell(v)+\ell(u)$.

Acknowledgments We thank P. Tingly and B. Webster for their kindness to explain us their works on convex preorders. We also thank B. Leclerc for many discussions around this subject. We thank Y. Kimura for fruitful communication. Finally, we want to thank P. McNamara for discussions on the PBW bases.

\section{Preliminaries}

1.1. Quantum groups. Let $I$ be an index set. A Cartan datum $\left(\mathrm{A}, \mathrm{P}, \Pi, \Pi^{\vee},(\cdot, \cdot)\right)$ consists of

(1) a free abelian group $\mathrm{P}$, called the weight lattice,

(2) $\Pi=\left\{\alpha_{i} \mid i \in I\right\} \subset \mathrm{P}$, called the set of simple roots,

(3) $\Pi^{\vee}=\left\{h_{i} \mid i \in I\right\} \subset \mathrm{P}^{\vee}:=\operatorname{Hom}(\mathrm{P}, \mathbb{Z})$, called the set of simple coroots,

(4) a $\mathbb{Q}$-valued symmetric bilinear form $(\cdot, \cdot)$ on $\mathrm{P}$ which satisfies

(a) $\left(\alpha_{i}, \alpha_{i}\right) \in 2 \mathbb{Z}_{>0}$ for $i \in I$,

(b) $\left\langle h_{i}, \lambda\right\rangle=\frac{2\left(\alpha_{i}, \lambda\right)}{\left(\alpha_{i}, \alpha_{i}\right)}$ for $i \in I$ and $\lambda \in \mathrm{P}$,

(c) $\mathrm{A}:=\left(\left\langle h_{i}, \alpha_{j}\right\rangle\right)_{i, j \in I}$ is a generalized Cartan matrix, i.e., $\left\langle h_{i}, \alpha_{i}\right\rangle=2$ for any $i \in I$ and $\left\langle h_{i}, \alpha_{j}\right\rangle \in \mathbb{Z}_{\leq 0}$ if $i \neq j$,

(d) $\Pi$ is a linearly independent set,

(e) for each $i \in I$, there exists $\Lambda_{i} \in \mathrm{P}$ such that $\left\langle h_{j}, \Lambda_{i}\right\rangle=\delta_{i j}$ for any $j \in I$.

We denote by $\Delta_{+}$the set of positive roots and set

$$
\mathrm{Q}=\bigoplus_{i \in I} \mathbb{Z} \alpha_{i}, \quad \mathrm{Q}_{+}=\sum_{i \in I} \mathbb{Z}_{\geq 0} \alpha_{i}, \quad \mathrm{Q}_{-}=\sum_{i \in I} \mathbb{Z}_{\leq 0} \alpha_{i}
$$

We write $\operatorname{ht}(\beta)=\sum_{i \in I} k_{i}$ and $\operatorname{supp}(\beta)=\left\{i \in I \mid k_{i} \neq 0\right\}$ for $\beta=\sum_{i \in I} k_{i} \alpha_{i} \in \mathrm{Q}_{+}$. 
The Weyl group W associated with the Cartan datum is the subgroup of Aut(P) generated by $\left\{s_{i}\right\}_{i \in I}$ given by

$$
s_{i}(\lambda)=\lambda-\left\langle h_{i}, \lambda\right\rangle \alpha_{i} \quad \text { for } \lambda \in \mathrm{P} .
$$

For $w \in \mathrm{W}$, the expression $w=s_{i_{1}} \cdots s_{i_{\ell}}$ is called a reduced expression if $\ell$ is minimal among all such expressions, and we set $\ell(w):=\ell$. For $w, v \in \mathbf{W}$, we write $w \geq v$ if there is a reduced expression of $v$ which appears in a reduced expression $w$ as a subexpression.

Let $U_{q}(\mathfrak{g})$ be the quantum group associated with the Cartan datum, which is an associative algebra over $\mathbb{Q}(q)$ generated by $e_{i}, f_{i}(i \in I)$ and $q^{h}\left(h \in \mathrm{P}^{\vee}\right)$ with certain defining relations (see [2, Chap. 3] for details). For $\beta \in \mathbf{Q}, U_{q}(\mathfrak{g})_{\beta}$ is the weight space of $U_{q}(\mathfrak{g})$ with weight $\beta$ and we set $\operatorname{wt}(x)=\beta$ for $x \in U_{q}(\mathfrak{g})_{\beta}$. We denote by $U_{q}^{+}(\mathfrak{g})$ (resp. $\left.U_{q}^{-}(\mathfrak{g})\right)$ the subalgebra of $U_{q}(\mathfrak{g})$ generated by $e_{i}$ (resp. $f_{i}$ ) for $i \in I$. For $n \in \mathbb{Z}_{\geq 0}$ and $i \in I$, we set $e_{i}^{(n)}:=e_{i}^{n} /[n]_{i}$ ! and $f_{i}^{(n)}:=f_{i}^{n} /[n]_{i}$ !, where $q_{i}=q^{\left(\alpha_{i}, \alpha_{i}\right) / 2}$,

$$
[n]_{i}=\frac{q_{i}^{n}-q_{i}^{-n}}{q_{i}-q_{i}^{-1}} \quad \text { and } \quad[n]_{i} !=\prod_{k=1}^{n}[k]_{i} .
$$

We denote by $U_{\mathbb{Z}\left[q, q^{-1}\right]}^{-}(\mathfrak{g})$ the $\mathbb{Z}\left[q, q^{-1}\right]$-subalgebra of $U_{q}^{-}(\mathfrak{g})$ generated by $f_{i}^{(n)}$ for $i \in I$ and $n \in \mathbb{Z}_{\geq 0}$. Similarly we define $U_{\mathbb{Z}\left[q, q^{-1}\right]}^{+}(\mathfrak{g})$.

The definitions of the category $\mathcal{O}_{\text {int }}(\mathfrak{g})$ of integrable $U_{q}(\mathfrak{g})$-modules and crystal bases can be found in $[2,8]$. For a left $U_{q}(\mathfrak{g})$-module $M$, we denote by $M^{r}$ the right $U_{q}(\mathfrak{g})$ module $\left\{m^{r} \mid m \in M\right\}$ with the right actions defined by

$$
\left(m^{r}\right) x=(\varphi(x) m)^{r} \quad \text { for } m \in M \text { and } x \in U_{q}(\mathfrak{g}),
$$

where $\varphi$ is the $\mathbb{Q}(q)$-antiautomorphism of $U_{q}(\mathfrak{g})$ given by $\varphi\left(e_{i}\right)=f_{i}, \varphi\left(f_{i}\right)=e_{i}, \varphi\left(q^{h}\right)=$ $q^{h}$. We denote by $\mathcal{O}_{\text {int }}^{\mathrm{r}}(\mathfrak{g})$ the category of right integrable modules $M^{r}$ such that $M \in \mathcal{O}_{\text {int }}(\mathfrak{g})$.

For a dominant integral weight $\Lambda \in \mathrm{P}_{+}$, we denote by $V_{q}(\Lambda)$ the irreducible highest weight module with highest weight $\Lambda$. There is a unique non-degenerate symmetric bilinear form $($, ) such that

$$
\left(u_{\Lambda}, u_{\Lambda}\right)=1 \text { and }(x u, v)=(u, \varphi(x) v) \text { for } u, v \in V_{q}(\Lambda) \text { and } x \in U_{q}(\mathfrak{g}),
$$

where $u_{\Lambda}$ is the highest weight vector of $V_{q}(\Lambda)$. This bilinear form induces the nondegenerated bilinear form

$$
\langle,\rangle: V_{q}(\Lambda)^{r} \otimes_{U_{q}(\mathfrak{g})} V_{q}(\Lambda) \longrightarrow \mathbb{Q}(q)
$$

defined by $\left\langle u^{r}, v\right\rangle=(u, v)$ for $u, v \in V_{q}(\mathfrak{g})$. 
It was known that every integrable $U_{q}(\mathfrak{g})$-module in $\mathcal{O}_{\text {int }}(\mathfrak{g})$ has a crystal base. In particular, the irreducible highest weight modules $V_{q}(\Lambda)$ for $\Lambda \in \mathrm{P}_{+}$have crystal bases. Let us recall the notion of a crystal.

Definition 1.1. A crystal is a set $B$ together with maps wt $: B \rightarrow \mathrm{P}, \varphi_{i}, \varepsilon_{i}: B \rightarrow$ $\mathbb{Z} \cup\{\infty\}$, and $\widetilde{e}_{i}, \widetilde{f}_{i}: B \rightarrow B \cup\{0\}$ which satisfy the following:

(a) $\varphi_{i}(b)=\varepsilon_{i}(b)+\left\langle h_{i}, \operatorname{wt}(b)\right\rangle$,

(b) $\operatorname{wt}\left(\widetilde{e}_{i} b\right)=\operatorname{wt}(b)+\alpha_{i}, \operatorname{wt}\left(\widetilde{f}_{i} b\right)=\operatorname{wt}(b)-\alpha_{i}$ if $\widetilde{e}_{i} b$ and $\widetilde{f}_{i} b \in B$,

(c) for $b, b^{\prime} \in B$ and $i \in I, b^{\prime}=\widetilde{e}_{i} b$ if and only if $b=\widetilde{f}_{i} b^{\prime}$,

(d) for $b \in B$, if $\varphi_{i}(b)=-\infty$, then $\widetilde{e}_{i} b=\widetilde{f}_{i} b=0$,

(e) if $b \in B$ and $\widetilde{e}_{i} b \in B$, then $\varepsilon_{i}\left(\widetilde{e}_{i} b\right)=\varepsilon_{i}(b)-1$ and $\varphi_{i}\left(\widetilde{e}_{i} b\right)=\varphi_{i}(b)+1$,

(f) if $b \in B$ and $\widetilde{f}_{i} b \in B$, then $\varepsilon_{i}\left(\widetilde{f}_{i} b\right)=\varepsilon_{i}(b)+1$ and $\varphi_{i}\left(\widetilde{f}_{i} b\right)=\varphi_{i}(b)-1$.

We denote by $B(\infty)$ the crystal of $U_{q}^{-}(\mathfrak{g})$ in which $\varepsilon_{i}$ and $\varphi_{i}$ are given as

$$
\varepsilon_{i}(b)=\max \left\{k \geq 0 \mid \tilde{e}_{i}^{k} b \neq 0\right\}, \quad \varphi_{i}(b)=\varepsilon_{i}(b)+\left\langle h_{i}, \operatorname{wt}(b)\right\rangle .
$$

The $\mathbb{Q}(q)$-antiautomorphism $*$ of $U_{q}(\mathfrak{g})$ defined by

$$
e_{i}^{*}=e_{i}, \quad f_{i}^{*}=f_{i}, \quad\left(q^{h}\right)^{*}=q^{-h}
$$

gives another crystal structure with $\widetilde{e}_{i}^{*}, \widetilde{f}_{i}^{*}, \varepsilon_{i}^{*}, \varphi_{i}^{*}$ on $U_{q}^{-}(\mathfrak{g})$. For $b \in B(\infty)$ and $i \in I$, we set

$$
\widetilde{e}_{i}^{\max }(b)=\widetilde{e}_{i}^{\varepsilon_{i}(b)}(b), \quad \widetilde{e}_{i}^{* \max }(b)=\widetilde{e}_{i}^{* \varepsilon_{i}^{*}(b)}(b) .
$$

We define the quantum coordinate ring $A_{q}(\mathfrak{g})$ by

$A_{q}(\mathfrak{g})=\left\{u \in U_{q}(\mathfrak{g})^{*} \mid U_{q}(\mathfrak{g}) u\right.$ belongs to $\mathcal{O}_{\text {int }}(\mathfrak{g})$ and $u U_{q}(\mathfrak{g})$ belongs to $\left.\mathcal{O}_{\text {int }}^{\mathrm{r}}(\mathfrak{g})\right\}$, where $U_{q}(\mathfrak{g})^{*}=\operatorname{Hom}_{\mathbb{Q}(q)}\left(U_{q}(\mathfrak{g}), \mathbb{Q}(q)\right)$. Then we have the following.

Proposition $1.2\left(\left[9\right.\right.$, Proposition 7.2.2]). There is an isomorphism $\Phi$ of $U_{q}(\mathfrak{g})$-bimodules

$$
\Phi: \bigoplus_{\Lambda \in \mathrm{P}_{+}} V_{q}(\Lambda) \otimes_{\mathbb{Q}(q)} V_{q}(\Lambda)^{r} \stackrel{\sim}{\longrightarrow} A_{q}(\mathfrak{g})
$$

defined by $\Phi\left(u \otimes v^{r}\right)(x)=\left\langle v^{r}, x u\right\rangle$ for $v, u \in V_{q}(\Lambda)$ and $x \in U_{q}(g)$.

The tensor product $U_{q}^{+}(\mathfrak{g}) \otimes U_{q}^{+}(\mathfrak{g})$ has the algebra structure defined by

$$
\left(x_{1} \otimes x_{2}\right) \cdot\left(y_{1} \otimes y_{2}\right)=q^{-\left(\operatorname{wt}\left(x_{2}\right), \operatorname{wt}\left(y_{1}\right)\right)}\left(x_{1} y_{1} \otimes x_{2} y_{2}\right)
$$

for homogeneous elements $x_{1}, x_{2}, y_{1}, y_{2} \in U_{q}^{+}(\mathfrak{g})$. We define the $\mathbb{Q}(q)$-algebra homomorphism $\Delta_{\mathfrak{n}}: U_{q}^{+}(\mathfrak{g}) \longrightarrow U_{q}^{+}(\mathfrak{g}) \otimes U_{q}^{+}(\mathfrak{g})$ by

$$
\Delta_{\mathfrak{n}}\left(e_{i}\right)=e_{i} \otimes 1+1 \otimes e_{i} \quad \text { for } i \in I .
$$


We set

$$
A_{q}(\mathfrak{n})=\bigoplus_{\beta \in Q_{-}} A_{q}(\mathfrak{n})_{\beta}, \quad \text { where } A_{q}(\mathfrak{n})_{\beta}:=\operatorname{Hom}_{\mathbb{Q}(q)}\left(U_{q}^{+}(\mathfrak{g})_{-\beta}, \mathbb{Q}(q)\right) .
$$

Defining the bilinear from $\langle\cdot, \cdot\rangle:\left(A_{q}(\mathfrak{n}) \otimes A_{q}(\mathfrak{n})\right) \times\left(U_{q}^{+}(\mathfrak{g}) \otimes U_{q}^{+}(\mathfrak{g})\right)$ by

$$
\langle\psi \otimes \theta, x \otimes y\rangle=\theta(x) \psi(y),
$$

the multiplication on $A_{q}(\mathfrak{n})$ is given by

$$
(\psi \cdot \theta)(x)=\left\langle\psi \otimes \theta, \Delta_{\mathfrak{n}}(x)\right\rangle \quad \text { for } \psi, \theta \in A_{q}(\mathfrak{n}), x \in U_{q}^{+}(\mathfrak{g}) .
$$

This is called the unipotent quantum coordinate ring. Note that there is a $\mathbb{Q}(q)$-algebra isomorphism between $A_{q}(\mathfrak{n})$ and $U_{q}^{-}(\mathfrak{g})([7$, Lemma 8.2.2]).

We set

$$
A_{q}(\mathfrak{n})_{\mathbb{Z}\left[q, q^{-1}\right]}=\left\{\psi \in A_{q}(\mathfrak{n}) \mid \psi\left(U_{\mathbb{Z}\left[q, q^{-1}\right]}^{+}(\mathfrak{g})\right) \subset \mathbb{Z}\left[q, q^{-1}\right]\right\} .
$$

Let $e_{i}$ and $e_{i}^{*} \in \operatorname{End}\left(A_{q}(\mathfrak{n})\right)$ denote the endomorphisms induced by the right multiplication and the left multiplication of $e_{i}$ on $U_{q}^{+}(\mathfrak{g})$, respectively.

Lemma 1.3 ([7, Lemma 8.2.1]). For $u, v \in A_{q}(\mathfrak{n})$, we have

$$
e_{i}(u v)=\left(e_{i} u\right) v+q^{\left(\alpha_{i}, \mathrm{wt}(u)\right)} u\left(e_{i} v\right) \quad \text { and } \quad e_{i}^{*}(u v)=u\left(e_{i}^{*} v\right)+q^{\left(\alpha_{i}, \mathrm{wt}(v)\right)}\left(e_{i}^{*} u\right) v .
$$

The algebra $A_{q}(\mathfrak{n})$ has the upper global basis

$$
\mathbf{B}^{\mathrm{up}}\left(A_{q}(\mathfrak{n})\right)=\left\{G^{\mathrm{up}}(b) \mid b \in B\left(A_{q}(\mathfrak{n})\right)\right\},
$$

where $B\left(A_{q}(\mathfrak{n})\right)$ is the crystal basis of $A_{q}(\mathfrak{n})$ which is isomorphic to $B(\infty)$.

Lemma 1.4 ([9, Lemma 5.1.1]). For $b \in B\left(A_{q}(\mathfrak{n})\right)$, we have

$$
e_{i}^{(m)} G^{\mathrm{up}}(b)=G^{\mathrm{up}}\left(\tilde{e}_{i}^{m} b\right), \quad e_{i}^{*(n)} G^{\mathrm{up}}(b)=G^{\mathrm{up}}\left(\tilde{e}_{i}^{* n} b\right),
$$

where $m=\varepsilon_{i}(b)$ and $n=\varepsilon_{i}^{*}(b)$.

The homomorphism $p_{\mathfrak{n}}: A_{q}(\mathfrak{g}) \rightarrow A_{q}(\mathfrak{n})$ induced by $U_{q}^{+}(\mathfrak{g}) \rightarrow U_{q}(\mathfrak{g})$ is given by

$$
\left\langle p_{\mathfrak{n}}(\psi), x\right\rangle=\psi(x) \quad \text { for any } x \in U_{q}^{+}(\mathfrak{g}) \text {. }
$$

Definition 1.5. For $\Lambda \in \mathrm{P}_{+}$and $\mu, \zeta \in \mathrm{W} \Lambda$, we define a (generalized) quantum minor $\Delta(\mu, \zeta)$ and a unipotent quantum minor $D(\mu, \zeta)$ as follows:

$$
\Delta(\mu, \zeta):=\Phi\left(u_{\mu} \otimes u_{\zeta}^{r}\right) \in A_{q}(\mathfrak{g}), \quad D(\mu, \zeta):=p_{\mathfrak{n}}(\Delta(\mu, \zeta)) \in A_{q}(\mathfrak{n}) .
$$

Recall that for $\lambda, \mu \in \mathrm{P}$, we write $\lambda \preceq \mu$ if there exists a sequence of real positive $\operatorname{roots} \beta_{k}(1 \leq k \leq \ell)$ such that $\lambda=s_{\beta_{\ell}} \cdots s_{\beta_{1}} \mu$ and $\left(\beta_{k}, s_{\beta_{k-1}} \cdots s_{\beta_{1}} \mu\right)>0$ for $1 \leq k \leq \ell$. When $\Lambda \in \mathrm{P}_{+}$and $\lambda, \mu \in \mathrm{W} \Lambda$, the relation $\lambda \preceq \mu$ holds if and only if there exist $w$, $v \in \mathrm{W}$ such that $\lambda=w \Lambda, \mu=v \Lambda$ and $v \leq w$. 
Lemma 1.6 ([7, Lemma 9.1.1, Corollary 9.1.3, Lemma 9.1.4]). (i) For $\Lambda \in \mathrm{P}_{+}$and $\mu, \zeta \in \mathrm{W} \Lambda, D(\mu, \zeta)$ is a member of the upper global basis of $A_{q}(\mathfrak{n})$ if $\mu \preceq \zeta$. Otherwise, $D(\mu, \zeta)$ is zero.

(ii) Let $\Lambda, \Lambda^{\prime} \in \mathrm{P}_{+}$and $w, v \in \mathrm{W}$ with $v \leq w$. Then

$$
D(w \Lambda, v \Lambda) D\left(w \Lambda^{\prime}, v \Lambda^{\prime}\right)=q^{-\left(v \Lambda, v \Lambda^{\prime}-w \Lambda^{\prime}\right)} D\left(w\left(\Lambda+\Lambda^{\prime}\right), v\left(\Lambda+\Lambda^{\prime}\right)\right) .
$$

Lemma 1.7 ([7, Lemma 9.1.5]). Let $\Lambda \in \mathrm{P}_{+}$and $\mu, \zeta \in \mathrm{W} \Lambda$ with $\mu \preceq \zeta$ and $i \in I$.

(i) If $n:=\left\langle h_{i}, \mu\right\rangle \geq 0$, then

$$
\varepsilon_{i}(D(\mu, \zeta))=0 \text { and } e_{i}^{(n)} D\left(s_{i} \mu, \zeta\right)=D(\mu, \zeta) .
$$

(ii) If $\left\langle h_{i}, \mu\right\rangle \leq 0$ and $s_{i} \mu \preceq \zeta$, then $\varepsilon_{i}(D(\mu, \zeta))=-\left\langle h_{i}, \mu\right\rangle$.

(iii) If $m:=-\left\langle h_{i}, \zeta\right\rangle \geq 0$, then

$$
\varepsilon_{i}^{*}(D(\mu, \zeta))=0 \text { and } e_{i}^{*(m)} D\left(\mu, s_{i} \zeta\right)=D(\mu, \zeta) .
$$

(iv) If $\left\langle h_{i}, \zeta\right\rangle \geq 0$ and $\mu \preceq s_{i} \zeta$, then $\varepsilon_{i}^{*}(D(\mu, \zeta))=\left\langle h_{i}, \zeta\right\rangle$.

1.2. Quiver Hecke algebras. Let $\mathbf{k}$ be a field. For $i, j \in I$, we choose polynomials $\mathcal{Q}_{i, j}(u, v) \in \mathbf{k}[u, v]$ such that

(a) $\mathcal{Q}_{i, j}(u, v)=\mathcal{Q}_{j, i}(v, u)$,

(b) it is of the form

$$
\mathcal{Q}_{i, j}(u, v)= \begin{cases}\sum_{p\left(\alpha_{i}, \alpha_{i}\right)+q\left(\alpha_{j}, \alpha_{j}\right)=-2\left(\alpha_{i}, \alpha_{j}\right)} t_{i, j ; p, q} u^{p} v^{q} & \text { if } i \neq j, \\ 0 & \text { if } i=j,\end{cases}
$$

where $t_{i, j ;-a_{i j}, 0} \in \mathbf{k}^{\times}$.

We set

$$
\overline{\mathcal{Q}}_{i, j}(u, v, w)=\frac{\mathcal{Q}_{i, j}(u, v)-\mathcal{Q}_{i, j}(w, v)}{u-w} \in \mathbf{k}[u, v, w] .
$$

For $\beta \in \mathbf{Q}_{+}$with $\operatorname{ht}(\beta)=n$, set

$$
I^{\beta}:=\left\{\nu=\left(\nu_{1}, \ldots, \nu_{n}\right) \in I^{n} \mid \sum_{k=1}^{n} \alpha_{\nu_{k}}=\beta\right\} .
$$

Note that the symmetric group $\mathfrak{S}_{n}=\left\langle s_{k} \mid k=1, \ldots, n-1\right\rangle$ acts on $I^{\beta}$ by place permutations.

Definition 1.8. For $\beta \in \mathrm{Q}_{+}$, the quiver Hecke algebra $R(\beta)$ associated with $\mathrm{A}$ and $\left(\mathcal{Q}_{i, j}(u, v)\right)_{i, j \in I}$ is the $\mathbf{k}$-algebra generated by

$$
\left\{e(\nu) \mid \nu \in I^{\beta}\right\},\left\{x_{k} \mid 1 \leq k \leq n\right\},\left\{\tau_{l} \mid 1 \leq l \leq n-1\right\}
$$


satisfying the following defining relations:

$$
\begin{aligned}
& e(\nu) e\left(\nu^{\prime}\right)=\delta_{\nu, \nu^{\prime}} e(\nu), \sum_{\nu \in I^{\beta}} e(\nu)=1, x_{k} e(\nu)=e(\nu) x_{k}, x_{k} x_{l}=x_{l} x_{k}, \\
& \tau_{l} e(\nu)=e\left(s_{l}(\nu)\right) \tau_{l}, \tau_{k} \tau_{l}=\tau_{l} \tau_{k} \text { if }|k-l|>1, \\
& \tau_{k}^{2} e(\nu)=\mathcal{Q}_{\nu_{k}, \nu_{k+1}}\left(x_{k}, x_{k+1}\right) e(\nu), \\
& \left(\tau_{k} x_{l}-x_{s_{k}(l)} \tau_{k}\right) e(\nu)= \begin{cases}-e(\nu) & \text { if } l=k \text { and } \nu_{k}=\nu_{k+1}, \\
e(\nu) & \text { if } l=k+1 \text { and } \nu_{k}=\nu_{k+1}, \\
0 & \text { otherwise, }\end{cases} \\
& \left(\tau_{k+1} \tau_{k} \tau_{k+1}-\tau_{k} \tau_{k+1} \tau_{k}\right) e(\nu) \\
& = \begin{cases}\overline{\mathcal{Q}}_{\nu_{k}, \nu_{k+1}}\left(x_{k}, x_{k+1}, x_{k+2}\right) e(\nu) & \text { if } \nu_{k}=\nu_{k+2}, \\
0 & \text { otherwise. }\end{cases}
\end{aligned}
$$

The algebra $R(\beta)$ has the $\mathbb{Z}$-graded algebra structure defined by

$$
\operatorname{deg}(e(\nu))=0, \quad \operatorname{deg}\left(x_{k} e(\nu)\right)=\left(\alpha_{\nu_{k}}, \alpha_{\nu_{k}}\right), \quad \operatorname{deg}\left(\tau_{l} e(\nu)\right)=-\left(\alpha_{\nu_{l}}, \alpha_{\nu_{l+1}}\right) .
$$

We denote by $R(\beta)$-Mod the category of graded $R(\beta)$-modules with degree preserving homomorphisms. We set $R(\beta)$-mod to be the full subcategory of $R(\beta)$-Mod consisting of the modules which are finite-dimensional over $\mathbf{k}$, and set $R(\beta)$-proj to be the full subcategory of $R(\beta)$-Mod consisting of finitely generated projective graded $R(\beta)$ modules. We denote $R$-Mod $:=\bigoplus_{\beta \in Q_{+}} R(\beta)$-Mod, $R$-proj $:=\bigoplus_{\beta \in Q_{+}} R(\beta)$-proj, and $R$-mod $:=\bigoplus_{\beta \in Q_{+}} R(\beta)$-mod. The objects of $R$-Mod are sometimes called $R$-modules. For simplicity, we write "a module" instead of "a graded module".

We denote by $q$ the grading shift functor, i.e. $(q M)_{k}=M_{k-1}$ for a graded module $M=\bigoplus_{k \in \mathbb{Z}} M_{k}$. For $M \in R(\beta)$-mod, the $q$-character of $M$ is given by

$$
\operatorname{ch}_{q}(M):=\sum_{\nu \in I^{\beta}} \operatorname{dim}_{q}(e(\nu) M) \nu,
$$

where $\operatorname{dim}_{q} V:=\sum_{k \in \mathbb{Z}} \operatorname{dim}\left(V_{k}\right) q^{k}$ for a graded vector space $V=\bigoplus_{k \in \mathbb{Z}} V_{k}$.

For $R(\beta)$-modules $M$ and $N, \operatorname{Hom}_{R(\beta)}(M, N)$ denotes the space of degree preserving module homomorphisms. We set $\operatorname{deg}(f)=k$ for $f \in \operatorname{Hom}_{R(\beta)}\left(q^{k} M, N\right)$, and define

$$
\operatorname{Hom}_{R(\beta)}(M, N):=\bigoplus_{k \in \mathbb{Z}} \operatorname{Hom}_{R(\beta)}\left(q^{k} M, N\right)
$$

We sometimes write $R$ for $R(\beta)$ in $\operatorname{Hom}_{R(\beta)}(M, N)$ and $\operatorname{Hom}_{R(\beta)}(M, N)$ for simplicity. 
For $\nu \in I^{\beta}$ and $\nu^{\prime} \in I^{\beta^{\prime}}$, let $e\left(\nu, \nu^{\prime}\right)$ be the idempotent corresponding to the concatenation $\nu * \nu^{\prime}$ of $\nu$ and $\nu^{\prime}$, and set

$$
e\left(\beta, \beta^{\prime}\right):=\sum_{\nu \in I^{\beta}, \nu^{\prime} \in I^{\beta^{\prime}}} e\left(\nu, \nu^{\prime}\right) \in R\left(\beta+\beta^{\prime}\right) .
$$

We define

$$
\operatorname{Res}_{\beta, \beta^{\prime}} L:=e\left(\beta, \beta^{\prime}\right) L \quad \text { for } L \in R\left(\beta+\beta^{\prime}\right) \text {-Mod, }
$$

and, for $M \in R(\beta)$-Mod and $N \in R\left(\beta^{\prime}\right)$-Mod,

$$
M \circ N:=R\left(\beta+\beta^{\prime}\right) e\left(\beta, \beta^{\prime}\right) \otimes_{R(\beta) \otimes R\left(\beta^{\prime}\right)}(M \otimes N) .
$$

Note that $\operatorname{Res}_{\beta, \beta^{\prime}} L$ is an $R(\beta) \otimes R\left(\beta^{\prime}\right)$-module and $M \circ N$ is an $R\left(\beta+\beta^{\prime}\right)$-module. We denote by $M \nabla N$ the head of $M \circ N$ and by $M \Delta N$ the socle of $M \circ N$. For $i \in I$, the functors $E_{i}$ and $F_{i}$ are defined by

$$
\begin{gathered}
E_{i}: R\left(\beta+\alpha_{i}\right) \text {-Mod } \rightarrow R(\beta)-\operatorname{Mod} \\
F_{i}: R(\beta) \text {-Mod } \rightarrow R\left(\beta+\alpha_{i}\right) \text {-Mod }
\end{gathered}
$$

by

$$
E_{i}(N)=e\left(\alpha_{i}, \beta\right) N \quad \text { and } \quad F_{i}(M)=R\left(\alpha_{i}\right) \circ M
$$

for $N \in R\left(\beta+\alpha_{i}\right)$-Mod and $M \in R(\beta)$-Mod. For $i \in I$ and $n \in \mathbb{Z}_{>0}$, let $L(i)$ be the simple $R\left(\alpha_{i}\right)$-module concentrated on degree zero and $P\left(i^{(n)}\right)$ the indecomposable projective $R\left(n \alpha_{i}\right)$-module whose head is isomorphic to $L\left(i^{n}\right):=q_{i}^{\frac{n(n+1)}{2}} L(i)^{{ }^{\circ} n}$. Then, for $M \in R(\beta)$-Mod, we define

$$
F_{i}^{(n)} M:=P\left(i^{(n)}\right) \circ M, \quad E_{i}^{(n)} M:=\operatorname{Hom}_{R\left(n \alpha_{i}\right)}\left(P\left(i^{(n)}\right), e\left(n \alpha_{i}, \beta-n \alpha_{i}\right) M\right) .
$$

For $\beta \in \mathbf{Q}_{+}$and $M \in R(\beta)$-Mod, we define

$$
\operatorname{wt}(M)=-\beta
$$

and, for $i \in I$ and a self-dual simple $R(\beta)$-module $M$,

$$
\begin{aligned}
\varepsilon_{i}(M)= & \max \left\{k \geq 0 \mid E_{i}^{k} M \neq 0\right\}, \quad \varphi_{i}(M)=\varepsilon_{i}(M)+\left\langle h_{i}, \operatorname{wt}(M)\right\rangle, \\
\widetilde{E}_{i}(M)= & \text { the self-dual simple module being isomorphic to } \operatorname{soc}\left(E_{i} M\right) \\
& \text { up to a grading shift, } \\
\widetilde{F}_{i}(M)= & \text { the self-dual simple module being isomorphic to } L(i) \nabla M \\
& \text { up to a grading shift. }
\end{aligned}
$$

Then the set of the isomorphism classes of self-dual simple $R$-modules together with the quintuple (wt, $\varepsilon_{i}, \varphi_{i}, \widetilde{E}_{i}, \widetilde{F}_{i}$ ) forms a crystal which is isomorphic to the crystal 
$B(\infty)([16])$. We sometimes ignore the grading shift when using $\widetilde{E}_{i}$ and $\widetilde{F}_{i}$ if there is no afraid of confusion. The trivial $R(0)$-module of degree 0 is denoted by 1 . We remark that, for a simple $R$-module $M$,

$$
\widetilde{E}_{i}^{m} M \simeq E_{i}^{(m)} M
$$

when $m=\varepsilon_{i}(M)([13$, Lemma 3.8]).

We also can define $E_{i}^{*}, F_{i}^{*}, \widetilde{E}_{i}^{*}, \widetilde{F}_{i}^{*}$, etc. in the same manner as above if we replace the roles of $e\left(\alpha_{i}, \beta\right)$ and $R\left(\alpha_{i}\right) \circ$ - with the ones of $e\left(\beta, \alpha_{i}\right)$ and $-\circ R\left(\alpha_{i}\right)$.

We denote by $K_{0}\left(R\right.$-proj) and $K_{0}(R$-mod $)$ the Grothendieck groups of $R$-proj and $R$-mod respectively. Then we have the categorification theorem for $U_{\mathbb{Z}\left[q, q^{-1}\right]}^{-}(\mathfrak{g})$ and $A_{q}(\mathfrak{n})_{\mathbb{Z}\left[q, q^{-1}\right]}$.

Theorem 1.9 ([13, 14, 21]). There exist isomorphisms of $\mathbb{Z}\left[q, q^{-1}\right]$-bialgebras

$$
K_{0}(R-\text { proj }) \simeq U_{\mathbb{Z}\left[q, q^{-1}\right]}^{-}(\mathfrak{g}) \quad \text { and } \quad K_{0}(R \text {-mod }) \simeq A_{q}(\mathfrak{n})_{\mathbb{Z}\left[q, q^{-1}\right]}
$$

Let $\lambda \in \Lambda-\mathrm{Q}_{+}$and set $\beta=\Lambda-\lambda, n=\operatorname{ht}(\beta)$. We define the cyclotomic quiver Hecke algebra

$$
R^{\Lambda}(\lambda):=\frac{R(\beta)}{R(\beta) a_{\Lambda}\left(x_{n}\right) R(\beta)},
$$

where $a^{\Lambda}\left(x_{n}\right)=\sum_{\nu \in I^{\beta}} x_{n}^{\left\langle h_{\nu_{n}}, \Lambda\right\rangle} e(\nu)$.

We denote by $R^{\Lambda}(\lambda)$-Mod the category of graded $R^{\Lambda}(\lambda)$-modules. We also denote by $R^{\Lambda}(\lambda)$-proj and $R^{\Lambda}(\lambda)$-mod the category of finitely generated projective graded $R^{\Lambda}(\lambda)$ modules and the category of graded $R^{\Lambda}(\lambda)$-modules which are finite-dimensional over $\mathbf{k}$, respectively. Their morphisms are homomorphisms homogeneous of degree zero. We write $R^{\Lambda}$-proj $:=\bigoplus_{\beta \in Q_{+}} R^{\Lambda}(\Lambda-\beta)$-proj, $R^{\Lambda}$-mod $:=\bigoplus_{\beta \in Q_{+}} R^{\Lambda}(\Lambda-\beta)$-mod, etc.

We define the functors

$$
\begin{aligned}
& F_{i}^{\Lambda}: R^{\Lambda}(\lambda) \text {-Mod } \rightarrow R^{\Lambda}\left(\lambda-\alpha_{i}\right) \text {-Mod }, \\
& E_{i}^{\Lambda}: R^{\Lambda}(\lambda)-\operatorname{Mod} \rightarrow R^{\Lambda}\left(\lambda+\alpha_{i}\right) \text {-Mod }
\end{aligned}
$$

by

$$
\begin{aligned}
& F_{i}^{\Lambda} M=R^{\Lambda}\left(\lambda-\alpha_{i}\right) e\left(\alpha_{i}, \beta\right) \otimes_{R^{\Lambda}(\lambda)} M, \\
& E_{i}^{\Lambda} M=e\left(\alpha_{i}, \beta-\alpha_{i}\right) M
\end{aligned}
$$

for $M \in R^{\Lambda}(\lambda)$-Mod, where $\beta=\Lambda-\lambda \in \mathrm{Q}_{+}$. They are exact functors, and they give a categorification of $V_{q}(\Lambda)$. 
Theorem 1.10 ([4, Theorem 6.2]). For $\Lambda \in \mathrm{P}^{+}$, there exist $U_{\mathbb{Z}\left[q, q^{-1}\right]}(\mathfrak{g})$-module isomorphisms

$$
K_{0}\left(R^{\Lambda}-\operatorname{proj}\right) \simeq V_{\mathbb{Z}\left[q, q^{-1}\right]}(\Lambda), \quad K_{0}\left(R^{\Lambda}-\bmod \right) \simeq V_{\mathbb{Z}\left[q, q^{-1}\right]}(\Lambda)^{\vee} .
$$

For $n \in \mathbb{Z}_{\geq 0}$, the algebra $R\left(n \alpha_{i}\right)$ acts on $\left(F_{i}^{\Lambda}\right)^{n}$ and $\left(E_{i}^{\Lambda}\right)^{n}$ and we define

$$
\begin{aligned}
& F_{i}^{\Lambda(n)} M=\operatorname{Hom}_{R\left(n \alpha_{i}\right)}\left(P\left(i^{(n)}\right),\left(F_{i}^{\Lambda}\right)^{n} M\right), \\
& E_{i}^{\Lambda(n)} M=\operatorname{Hom}_{R\left(n \alpha_{i}\right)}\left(P\left(i^{(n)}\right),\left(E_{i}^{\Lambda}\right)^{n} M\right) .
\end{aligned}
$$

Then we obtain exact functors:

$$
\begin{aligned}
& F_{i}^{\Lambda(n)}: R^{\Lambda}(\lambda) \text {-Mod } \rightarrow R^{\Lambda}\left(\lambda-n \alpha_{i}\right) \text {-Mod }, \\
& E_{i}^{\Lambda(n)}: R^{\Lambda}(\lambda) \text {-Mod } \rightarrow R^{\Lambda}\left(\lambda+n \alpha_{i}\right) \text {-Mod. }
\end{aligned}
$$

Then the following lemma is an easy consequence of the theory of $\mathfrak{s l}_{2}$-categorification due to Rouquier ([21]).

Lemma 1.11. Let $\Lambda \in \mathrm{P}^{+}$and $\lambda \in \mathrm{W} \Lambda$ such that $n:=\left\langle h_{i}, \lambda\right\rangle \geq 0$. Then we have category equivalences, quasi-inverse to each other:

$$
R^{\Lambda}(\lambda)-\operatorname{Mod} \frac{F_{i}^{\Lambda(n)}}{\underset{E_{i}^{\Lambda(n)}}{\rightleftarrows}} R^{\Lambda}\left(s_{i} \lambda\right)-\operatorname{Mod} .
$$

1.3. Convex preorders. In this subsection, we review the notion of convex preorders introduced in [24], and show some of their properties.

Definition 1.12. (i) $A$ preorder $\preceq$ on a set $X$ is a binary relation on $X$ satisfying (a) $x \preceq x$ for any $x \in X$,

(b) if $x \preceq y$ and $y \preceq z$ for $x, y, z \in X$, then $x \preceq z$.

(ii) A preorder $\preceq$ on a set $X$ is total if, for any pair $(x, y)$ of elements of $X$, we have either $x \preceq y$ or $y \preceq x$.

(iii) For a preorder $\preceq$, we say that $x$ and $y$ are $\preceq$-equivalent if $x \preceq y$ and $y \preceq x$. An equivalent class for $\preceq$ is called $\preceq$-equivalence class.

(iv) We write $x \prec y$ (resp. $x \succ y$ ) if $x \preceq y$ and $x \nsucceq y$ (resp. $x \succeq y$ and $x \npreceq y$ ).

(v) For subsets $A$ and $B$, we write $A \preceq B$ if $a \preceq b$ for any $a \in A$ and $b \in B$. We also define $A \prec B, A \succ B$, etc., in a similar manner.

For preorders $\preceq$ and $\preceq^{\prime}$ on $X$, we say $\preceq^{\prime}$ is a refinement of $\preceq$ if $x \prec y$ implies $x \prec^{\prime} y$. 
Definition 1.13. A face is a decomposition of a subset $X$ of an $\mathbb{R}$-vector space into three disjoint subsets $X=A_{-} \sqcup A_{0} \sqcup A_{+}$such that

$$
\begin{aligned}
& \left(\operatorname{span}_{\mathbb{R}_{\geq 0}} A_{+}+\operatorname{span}_{\mathbb{R}} A_{0}\right) \cap \operatorname{span}_{\mathbb{R}_{\geq 0}} A_{-}=\{0\}, \\
& \left(\operatorname{span}_{\mathbb{R}_{\geq 0}} A_{-}+\operatorname{span}_{\mathbb{R}} A_{0}\right) \cap \operatorname{span}_{\mathbb{R}_{\geq 0}} A_{+}=\{0\},
\end{aligned}
$$

where $\operatorname{span}_{\mathbb{R}} S$ is the $\mathbb{R}$-vector space spanned by $S$ and $\operatorname{span}_{\mathbb{R}_{>0}} S$ is the subset of $\operatorname{span}_{\mathbb{R}} S$ whose elements are linear combinations of $S$ with non-negative coefficients.

Note that $\operatorname{span}_{\mathbb{R}} \emptyset=\operatorname{span}_{\mathbb{R}_{>0}} \emptyset=\{0\}$ by the definition. One can prove the following lemma easily.

Lemma 1.14. Let $\left(A_{-}, A_{0}, A_{+}\right)$be a decomposition of a subset $X$ in an $\mathbb{R}$-vector space. Then the following are equivalent:

(a) the triple $\left(A_{-}, A_{0}, A_{+}\right)$is a face,

(b) it satisfies

$$
\begin{aligned}
& \operatorname{span}_{\mathbb{R}_{\geq 0}}\left(A_{+} \cup A_{0}\right) \cap \operatorname{span}_{\mathbb{R}_{\geq 0}}\left(A_{-} \cup A_{0}\right)=\operatorname{span}_{\mathbb{R}_{\geq 0}} A_{0}, \\
& \operatorname{span}_{\mathbb{R}_{\geq 0}} A_{+} \cap \operatorname{span}_{\mathbb{R}} A_{0}=\operatorname{span}_{\mathbb{R}_{\geq 0}} A_{-} \cap \operatorname{span}_{\mathbb{R}} A_{0}=\{0\},
\end{aligned}
$$

(c) for any $x_{ \pm} \in \operatorname{span}_{\mathbb{R}_{\geq 0}} A_{ \pm}$, if $x_{-}-x_{+} \in \operatorname{span}_{\mathbb{R}} A_{0}$, then $x_{-}=x_{+}=0$.

Note that Lemma 1.14 tells that our definition of a face of $\Delta_{+}$is equivalent to that given in [20].

Remark 1.15. Let $\left(A_{-}, A_{0}, A_{+}\right)$be a decomposition of a subset $X$ in an $\mathbb{R}$-vector space. If there exists a linear functional $f$ such that

$$
A_{-} \backslash\{0\} \subset f^{-1}\left(\mathbb{R}_{<0}\right), \quad A_{0} \subset f^{-1}(0), \quad A_{+} \backslash\{0\} \subset f^{-1}\left(\mathbb{R}_{>0}\right),
$$

then the triple $\left(A_{-}, A_{0}, A_{+}\right)$is a face. Conversely, if $\left(A_{-}, A_{0}, A_{+}\right)$is a face and $A_{-}$and $A_{+}$are finite subsets, then there exists such a linear functional $f$.

Definition 1.16. Let $V$ be an $\mathbb{R}$-vector space and let $X$ be a subset of $V \backslash\{0\}$.

(i) A convex preorder $\preceq$ on $X$ is a total preorder on $X$ such that, for any $\preceq$ equivalence class $\mathcal{C}$, the triple $(\{x \in X \mid x \prec \mathcal{C}\}, \mathcal{C},\{x \in X \mid x \succ \mathcal{C}\})$ is a face.

(ii) A convex preorder $\preceq$ on $X$ is called a convex order if every $\preceq$-equivalence class is of the form $X \cap l$ for some line $l$ in $V$ through the origin.

Note that a convex order is not an order on $X$. It is an order on the image of $X$ by the projection $V \backslash\{0\} \rightarrow(V \backslash\{0\}) / \mathbb{R}^{\times}$.

The lemma below follows immediately from Definition 1.16 and Lemma 1.14. 
Lemma 1.17. Let $V$ be an $\mathbb{R}$-vector space and let $\preceq$ be a convex preorder on $X \subset$ $V \backslash\{0\}$.

(i) If $\beta, t \beta \in X$ for some $t \in \mathbb{R}$, then $\beta$ and $t \beta$ are in the same $\preceq$-equivalence class.

(ii) If $\alpha, \beta, \gamma \in X$ with $\alpha+\beta=\gamma$ and $\alpha \prec \gamma$, then $\gamma \prec \beta$.

(iii) If $\alpha, \beta, \gamma \in X$ with $\alpha+\beta=\gamma$ and $\gamma \prec \beta$, then $\alpha \prec \gamma$.

Lemma 1.18. Let $V$ be an $\mathbb{R}$-vector space and let $\preceq$ be a convex preorder on $X \subset$ $V \backslash\{0\}$ such that $\operatorname{span}_{\mathbb{R}_{\geq 0}} X \cap\left(-\operatorname{span}_{\mathbb{R}_{\geq 0}} X\right)=\{0\}$. Let $\mathcal{C}$ be $a \preceq$-equivalence class and set

$$
\mathcal{C}_{-}=\{x \in X \mid x \prec \mathcal{C}\} \quad \text { and } \quad \mathcal{C}_{+}=\{x \in X \mid x \succ \mathcal{C}\} .
$$

Let $\beta$ be an element of $\operatorname{span}_{\mathbb{R}_{>0}} \mathcal{C}^{\prime}$ for some $\preceq$-equivalence class $\mathcal{C}^{\prime}$.

(i) If $\beta$ can be written as $\beta=\beta_{1}+\beta_{2}$ for some $\beta_{1} \in \operatorname{span}_{\mathbb{R}_{>0}}\left(\mathcal{C}_{-} \cup \mathcal{C}\right)$ and some non-zero $\beta_{2} \in \operatorname{span}_{\mathbb{R}_{\geq 0}} \mathcal{C}_{-}$, then $\beta \in \operatorname{span}_{\mathbb{R}_{\geq 0}} \mathcal{C}_{-}$.

(ii) If $\beta$ can be written as $\beta=\beta_{1}+\beta_{2}$ for some $\beta_{1} \in \operatorname{span}_{\mathbb{R}_{\geq 0}}\left(\mathcal{C}_{+} \cup \mathcal{C}\right)$ and some non-zero $\beta_{2} \in \operatorname{span}_{\mathbb{R}_{\geq 0}} \mathcal{C}_{+}$, then $\beta \in \operatorname{span}_{\mathbb{R}_{\geq 0}} \mathcal{C}_{+}$.

Proof. (i) The element $\beta$ is contained in one of $\operatorname{span}_{\mathbb{R}_{\geq 0}} \mathcal{C}_{-}, \operatorname{span}_{\mathbb{R}_{\geq 0}} \mathcal{C}$ or $\operatorname{span}_{\mathbb{R}_{\geq 0}} \mathcal{C}_{+}$. If $\beta \in \operatorname{span}_{\mathbb{R}_{>0}} \mathcal{C}_{+}$, then $\beta \in \operatorname{span}_{\mathbb{R}_{\geq 0}}\left(\mathcal{C}_{+}\right) \cap\left(\operatorname{span}_{\mathbb{R}_{>0}}(\mathcal{C})+\operatorname{span}_{\mathbb{R}_{\geq 0}}\left(\mathcal{C}_{-}\right)\right)=\{0\}$. Suppose that $\beta \in \operatorname{span}_{\mathbb{R}_{\geq 0}} \mathcal{C}$. If we write $\beta_{1}=\beta_{1}^{-}+\beta_{1}^{0}$ for some $\beta_{1}^{-} \in \operatorname{span}_{\mathbb{R}_{\geq 0}} \mathcal{C}_{-}$and some $\beta_{1}^{0} \in \operatorname{span}_{\mathbb{R}_{>0}} \mathcal{C}$, then we have

$$
\beta=\beta_{1}+\beta_{2}=\beta_{1}^{-}+\beta_{1}^{0}+\beta_{2} .
$$

This tells that $\beta_{1}^{-}+\beta_{2}=\beta-\beta_{1}^{0} \in \operatorname{span}_{\mathbb{R}_{>0}} \mathcal{C}_{-} \cap \operatorname{span}_{\mathbb{R}} \mathcal{C}=\{0\}$. As $\operatorname{span}_{\mathbb{R}>0} X \cap$ $\left(-\operatorname{span}_{\mathbb{R}_{\geq 0}} X\right)=\{0\}$, we have $\beta_{2}=-\beta_{1}^{-}=0$, which is a contradiction to the fact that $\beta_{2} \neq 0$.

(ii) can be proved in the same manner as above.

Lemma 1.19. Let $n, m$ be positive integers such that $1<m \leq n$, and $V=\mathbb{R}^{n}$. We define

$$
H=\bigcup_{k=1}^{m}\left\{x=\left(x_{1}, \ldots, x_{n}\right) \in V \mid x_{j}=0 \text { for } 1 \leq j<k \text { and } x_{k}>0\right\} \subset V \backslash\{0\},
$$

and set $H_{+}=\left\{x \in H \mid x_{1}>0\right\}$ and $H_{0}=\left\{x \in H \mid x_{1}=0\right\}$. We define the total preorder $\preceq$ on $H$ as follows:

(a) $H_{0}$ is a $\preceq$-equivalence class, and $H_{+} \prec H_{0}$, 
(b) for $x, y \in H_{+}$, we define

$$
\begin{aligned}
x \prec y \Longleftrightarrow & \text { there exists } k \text { such that } 2 \leq k \leq m \text { and } \\
& \frac{x_{j}}{x_{1}}=\frac{y_{j}}{y_{1}} \text { for } 2 \leq j<k \text { and } \frac{x_{k}}{x_{1}}<\frac{y_{k}}{y_{1}} .
\end{aligned}
$$

Then $\preceq$ is a convex preorder on $H$. Moreover $\operatorname{dim} \operatorname{span}_{\mathbb{R}}(\mathcal{C})<\operatorname{dim} V$ for any $\preceq$ equivalence class $\mathcal{C}$.

Proof. It is obvious that $\preceq$ is a total preorder on $H$. Let $\mathcal{C}$ be a $\preceq$-equivalence class.

We set

$$
\mathcal{C}_{-}=\{x \in H \mid x \prec \mathcal{C}\} \quad \text { and } \quad \mathcal{C}_{+}=\{x \in H \mid x \succ \mathcal{C}\} .
$$

Let us show that

$$
\text { if } x_{ \pm} \in \operatorname{span}_{\mathbb{R}_{\geq 0}}\left(\mathcal{C}_{ \pm}\right) \text {satisfy } x_{-}-x_{+} \in \operatorname{span}_{\mathbb{R}}(\mathcal{C}) \text {, then } x_{-}=x_{+}=0 .
$$

Note that any equivalence class $\mathcal{C}$ is either $H_{0}$ or

$$
\mathcal{C}(\lambda):=\left\{x \in H \mid x_{k}=\lambda_{k} x_{1} \text { for } 2 \leq k \leq m\right\}
$$

for $\lambda=\left(\lambda_{2}, \ldots, \lambda_{m}\right) \in \mathbb{R}^{m-1}$.

If $\mathcal{C}=H_{0}$, then $\mathcal{C}_{-}=H_{+}=\operatorname{span}_{\mathbb{R}_{\geq 0}}\left(\mathcal{C}_{-}\right) \backslash\{0\}$, and $\mathcal{C}_{+}=\emptyset$. Hence (1.3) holds.

Suppose that $\mathcal{C}=\mathcal{C}(\lambda)$ for $\lambda=\left(\lambda_{2}, \ldots, \lambda_{m}\right) \in \mathbb{R}^{m-1}$. Then we have

$$
\begin{aligned}
& \mathcal{C}_{-}=\left\{\begin{array}{l|l}
x \in H_{+} & \begin{array}{l}
\text { there exists } k \text { such that } 2 \leq k \leq m, \\
x_{k}<\lambda_{k} x_{1} \text { and } x_{j}=\lambda_{j} x_{1} \text { for } 2 \leq j<k
\end{array}
\end{array}\right\} \\
& =\left\{x \in H \mid \begin{array}{l}
\text { there exists } k \text { such that } 2 \leq k \leq m, \\
x_{k}<\lambda_{k} x_{1} \text { and } x_{j}=\lambda_{j} x_{1} \text { for } 2 \leq j<k
\end{array}\right\} \\
& =\operatorname{span}_{\mathbb{R}_{\geq 0}}\left(\mathcal{C}_{-}\right) \backslash\{0\}, \\
& \mathcal{C}_{+}=H_{0} \cup\left\{x \in H_{+} \mid \begin{array}{l}
\text { there exists } k \text { such that } 2 \leq k \leq m, \\
x_{k}>\lambda_{k} x_{1} \text { and } x_{j}=\lambda_{j} x_{1} \text { for } 2 \leq j<k
\end{array}\right\} \\
& =\left\{\begin{array}{ll}
x \in H \mid & \begin{array}{l}
\text { there exists } k \text { such that } 2 \leq k \leq m, \\
x_{k}>\lambda_{k} x_{1} \text { and } x_{j}=\lambda_{j} x_{1} \text { for } 2 \leq j<k
\end{array}
\end{array}\right\} \\
& =\operatorname{span}_{\mathbb{R}_{\geq 0}}\left(\mathcal{C}_{+}\right) \backslash\{0\}, \\
& \operatorname{span}_{\mathbb{R}}(\mathcal{C})=\left\{x \in H \mid x_{k}=\lambda_{k} x_{1} \text { for } 2 \leq k \leq m\right\} .
\end{aligned}
$$

Then one can easily show that (1.3) holds.

Lemma 1.20. Let $V$ be an $\mathbb{R}$-vector space, and $\preceq$ a convex preorder on $X \subset V \backslash\{0\}$. For each $\preceq$-equivalence class $\mathcal{C}$ let $\preceq_{\mathcal{C}}$ be an arbitrary convex preorder on $\mathcal{C}$. Let $\preceq^{\prime}$ be the binary relation on $X$ defined as follows:

$x \preceq^{\prime} y$ if either $x \prec y$ or $x$ and $y$ belong to the same $\preceq$-equivalence class $\mathcal{C}$ and $x \preceq_{c} y$.

Then $\preceq^{\prime}$ is a convex preorder on $X$. 
Proof. It is obvious that $\preceq^{\prime}$ is a total preorder on $X$. We shall show that $\preceq^{\prime}$ is a convex preorder. Let $\mathcal{C}_{0}$ be a $\preceq^{\prime}$-equivalence class. Then $\mathcal{C}_{0}$ is contained in some $\preceq$-equivalence class $\mathcal{C}$. Then $A_{-}:=\left\{x \in X \mid x \prec^{\prime} \mathcal{C}_{0}\right\}$ is equal to $B_{-} \cup C_{-}$where $B_{-}=\{x \in X \mid x \prec \mathcal{C}\}$ and $C_{-}=\left\{x \in \mathcal{C} \mid x \prec_{\mathcal{C}} \mathcal{C}_{0}\right\}$. Similarly, we have $A_{+}=B_{+} \cup C_{+}$ where $A_{+}=\left\{x \in X \mid x \succ^{\prime} \mathcal{C}_{0}\right\}, B_{+}=\{x \in X \mid x \succ \mathcal{C}\}$ and $C_{+}=\left\{x \in \mathcal{C} \mid x \succ_{\mathcal{C}} \mathcal{C}_{0}\right\}$.

We take $x_{-} \in \operatorname{span}_{\mathbb{R}_{\geq 0}}\left(A_{-}\right)$and $x_{+} \in \operatorname{span}_{\mathbb{R}_{\geq 0}}\left(A_{+}\right)$such that $x_{-}-x_{+} \in \operatorname{span}_{\mathbb{R}}\left(\mathcal{C}_{0}\right)$. Then we can write

$$
x_{ \pm}=y_{ \pm}+z_{ \pm}
$$

for some $y_{ \pm} \in \operatorname{span}_{\mathbb{R}_{\geq 0}}\left(B_{ \pm}\right)$and some $z_{ \pm} \in \operatorname{span}_{\mathbb{R}_{\geq 0}}\left(C_{ \pm}\right)$. Since $x_{-}-x_{+}$and $z_{+}-z_{-}$ are contained in $\operatorname{span}_{\mathbb{R}}(\mathcal{C})$, we have

$$
y_{-}-y_{+}=x_{-}-x_{+}+z_{+}-z_{-} \subset \operatorname{span}_{\mathbb{R}}(\mathcal{C}),
$$

which implies $y_{-}=y_{+}=0$. Hence $z_{-}-z_{+} \in \operatorname{span}_{\mathbb{R}}\left(\mathcal{C}_{0}\right)$, which implies $z_{-}=z_{+}=0$.

Proposition 1.21. Let $V$ be a finite-dimensional $\mathbb{R}$-vector space, and let $X$ be a subset of $V \backslash\{0\}$ such that

$$
\operatorname{span}_{\mathbb{R}_{\geq 0}} X \cap\left(-\operatorname{span}_{\mathbb{R}_{\geq 0}} X\right)=\{0\} .
$$

For any convex preorder $\preceq$ on $X$, there exists a convex order on $X$ which refines $\preceq$.

Proof. Let $\preceq$ be a convex preorder on $X$. We set $n:=\operatorname{dim} V$.

We shall use induction on $n$. We first assume that $X$ itself is only one $\preceq$-equivalence

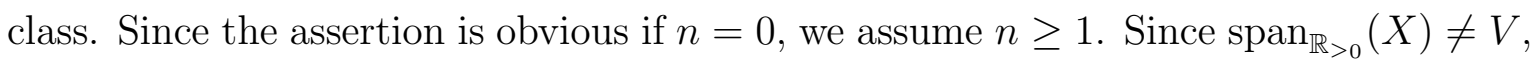
there exists a non-zero linear functional $f_{1}$ such that $X \subset f_{1}^{-1}\left(\mathbb{R}_{>0}\right)$. If $\operatorname{dim} V \geq 2$, there exists a linear functional $f_{2}$ such that $X \cap f_{1}^{-1}(0) \subset f_{2}^{-1}\left(\mathbb{R}_{\geq 0}\right)$ and $f_{1}$ and $f_{2}$ are linearly independent. Continuing this process to $n$, we can find an isomorphism $f: V \stackrel{\sim}{\rightarrow} \mathbb{R}^{n}$ such that $X \subset f^{-1}(H)$, where $H$ is given in Lemma 1.19. Let $\preceq$ be the convex preorder on $X$ induced by the convex preorder on $H$. $\operatorname{Since} \operatorname{dim} \operatorname{span}_{\mathbb{R}}(\mathcal{C})<n$ for any $\preceq$-equivalence class $\mathcal{C}$, the induction hypothesis implies that there exists a convex order $\preceq_{\mathcal{C}}$ on each $\mathcal{C}$. Thanks to Lemma 1.20, we obtain a desired convex order on $X$ by refining $\preceq$ by the $\preceq$ c's.

We now assume that $X$ is not a $\preceq$-equivalence class. Since $\operatorname{dim} \operatorname{span}_{\mathbb{R}} \mathcal{C}<n$ for any $\preceq$-equivalence class $\mathcal{C}$, every $\preceq$-equivalence class $\mathcal{C}$ has a convex order $\preceq_{\mathcal{C}}$ by the induction hypothesis. Using Lemma 1.20 , we obtain the assertion by refining $\preceq$ by $\preceq$ 's.

Definition 1.22. Let $V$ be a finite-dimensional $\mathbb{R}$-vector space. $A$ charge on $X \subset$ $V \backslash\{0\}$ is an $\mathbb{R}$-linear function $c: V \rightarrow \mathbb{C}$ such that

(a) $c(X)$ does not contain 0 , 
(b) $\operatorname{span}_{\mathbb{R}_{\geq 0}} c(X) \cap\left(-\operatorname{span}_{\mathbb{R}_{\geq 0}} c(X)\right)=\{0\}$.

As $\operatorname{span}_{\mathbb{R}_{\geq 0}} c(X) \backslash\{0\}$ is simply connected, we can define $\arg : c(X) \rightarrow \mathbb{R}$, which is unique up to a constant function. We set

$$
\arg _{c}:=\arg \circ c: X \longrightarrow \mathbb{R} .
$$

Lemma 1.23. Let $c: V \rightarrow \mathbb{C}$ be a charge on $X \subset V \backslash\{0\}$. Then the binary relation $\preceq_{c}$ on $X$ defined by

$$
x \preceq_{c} y \quad \text { if and only if } \quad \arg _{c} x \leq \arg _{c} y
$$

is a convex preorder on $X$.

Proof. It is obvious that $\preceq_{c}$ is a preorder on $X$. Without loss of generality, we may assume that $0 \leq \arg _{c} x \leq \pi$ for any $x \in X$ by Definition 1.22. We take a $\preceq$-equivalence class $\mathcal{C}$. Let $t:=\arg _{c} x$ for $x \in \mathcal{C}$, and

$$
\begin{aligned}
& \mathcal{C}_{-}:=\left\{y \in X \mid y \prec_{c} \mathcal{C}\right\}=\left\{y \in X \mid 0 \leq \arg _{c} y<t\right\}, \\
& \mathcal{C}_{+}:=\left\{z \in X \mid z \succ_{c} \mathcal{C}\right\}=\left\{z \in X \mid t<\arg _{c} z \leq \pi\right\} .
\end{aligned}
$$

Since $\arg _{c} y<t$ for $y \in \operatorname{span}_{\mathbb{R}_{\geq 0}} \mathcal{C}_{-} \backslash\{0\}$ and $\arg _{c} x=t$ for $x \in \operatorname{span}_{\mathbb{R}} \mathcal{C} \backslash\{0\}$, we have $\arg _{c}(y+x)<t$. This implies

$$
\left(\operatorname{span}_{\mathbb{R}_{\geq 0}} \mathcal{C}_{-}+\operatorname{span}_{\mathbb{R}} \mathcal{C}\right) \cap \operatorname{span}_{\mathbb{R}_{\geq 0}} \mathcal{C}_{+}=\{0\}
$$

In the same manner, one can prove

$$
\left(\operatorname{span}_{\mathbb{R}_{\geq 0}} \mathcal{C}_{+}+\operatorname{span}_{\mathbb{R}} \mathcal{C}\right) \cap \operatorname{span}_{\mathbb{R}_{\geq 0}} \mathcal{C}_{-}=\{0\}
$$

Therefore the triple $\left(\mathcal{C}_{-}, \mathcal{C}, \mathcal{C}_{+}\right)$is a face, which completes the proof.

The following proposition was informed to us by Peter McNamara.

Proposition 1.24. Let $\underline{w}=s_{i_{1}} s_{i_{2}} \cdots s_{i_{l}}$ be a reduced expression of $w \in \mathrm{W}$. We set $\beta_{k}:=s_{i_{1}} \cdots s_{i_{k-1}}\left(\alpha_{i_{k}}\right)$ so that $\Delta_{+} \cap w \Delta_{-}=\left\{\beta_{1}, \ldots, \beta_{l}\right\}$. Then there is a convex preorder $\preceq$ on $\Delta_{+}$such that

$$
\beta_{1} \prec \beta_{2} \prec \cdots \prec \beta_{l} \prec \gamma
$$

for any $\gamma \in \Delta_{+} \cap w \Delta_{+}$.

Proof. It is easy to see that $c:=\rho^{\vee}+\sqrt{-1} \rho^{\vee} \circ w^{-1}$ is a charge on $\Delta_{+}$. Here $\rho^{\vee} \in \mathrm{P}^{\vee}$ is defined by $\left\langle\rho^{\vee}, \alpha_{i}\right\rangle=1$ for $i \in I$. We define the total preorder on $\Delta_{+}$by

$$
\begin{aligned}
& \beta_{1} \prec \cdots \prec \beta_{l} \prec \beta \quad \text { for any } \beta \in \Delta_{+} \cap w \Delta_{+}, \\
& \beta \preceq \beta^{\prime} \Longleftrightarrow \arg _{c} \beta \leq \arg _{c} \beta^{\prime} \quad \text { for } \beta, \beta^{\prime} \in \Delta_{+} \cap w \Delta_{+} .
\end{aligned}
$$

We now show that $\preceq$ is a convex preorder on $\Delta_{+}$. 
For $k=0,1, \ldots, l$, we set $w_{k}:=s_{i_{1}} \cdots s_{i_{k}}$ and $\phi_{k}:=\rho^{\vee} \circ w_{k}^{-1}$. Since

$$
\beta_{j} \in w_{k} \Delta_{-} \text {for } 1 \leq j \leq k, \quad \beta_{j} \in w_{k} \Delta_{+} \text {for } k<j \leq l, \quad \Delta_{+} \cap w \Delta_{+} \subset w_{k} \Delta_{+},
$$

we have

$$
\begin{aligned}
& \left\{\beta_{1}, \ldots, \beta_{k}\right\} \subset \phi_{k}^{-1}\left(\mathbb{R}_{<0}\right), \\
& \left\{\beta_{k+1}, \ldots, \beta_{l}\right\} \cup\left(\Delta_{+} \cap w \Delta_{+}\right) \subset \phi_{k}^{-1}\left(\mathbb{R}_{>0}\right) .
\end{aligned}
$$

Letting

$$
\mathcal{C}_{-}:=\left\{\beta \in \Delta_{+} \mid \beta \prec \beta_{k}\right\}, \quad \mathcal{C}:=\left\{\beta_{k}\right\}, \quad \mathcal{C}_{+}:=\left\{\beta \in \Delta_{+} \mid \beta \succ \beta_{k}\right\},
$$

the hyperplane $\phi_{k-1}^{-1}(0)$ (resp. $\left.\phi_{k}^{-1}(0)\right)$ divides $\Delta_{+}$into $\mathcal{C}_{-}$and $\mathcal{C} \cup \mathcal{C}_{+}\left(\right.$resp. $\mathcal{C}_{-} \cup \mathcal{C}$ and $\left.\mathcal{C}_{+}\right)$. Thus, the triple $\left(\mathcal{C}_{-}, \mathcal{C}, \mathcal{C}_{+}\right)$satisfies the conditions in Lemma 1.14 , which tells the triple is a face.

We now take $\beta \in \Delta_{+} \cap w \Delta_{+}$and let $\mathcal{C}$ denote the $\preceq$-equivalence class containing $\beta$. Let $t=\rho^{\vee}\left(w^{-1} \beta\right) / \rho^{\vee}(\beta)$ and

$$
\mathcal{C}_{-}:=\left\{\gamma \in \Delta_{+} \mid \gamma \prec \mathcal{C}\right\}, \quad \mathcal{C}_{+}:=\left\{\gamma \in \Delta_{+} \mid \gamma \succ \mathcal{C}\right\}
$$

By the definition of $c$, we have

$$
\begin{aligned}
\mathcal{C}_{-} & =\left(\Delta_{+} \cap w \Delta_{-}\right) \cup\left\{\gamma \in \Delta_{+} \cap w \Delta_{+} \mid \rho^{\vee}\left(w^{-1} \gamma\right)<t \rho^{\vee}(\gamma)\right\} \\
& =\left\{\gamma \in \Delta_{+} \mid \rho^{\vee}\left(w^{-1} \gamma\right)<t \rho^{\vee}(\gamma)\right\}=\left\{\gamma \in \Delta_{+} \mid \gamma \prec_{c} \beta\right\}, \\
\mathcal{C} & =\left\{\gamma \in \Delta_{+} \mid \rho^{\vee}\left(w^{-1} \gamma\right)=t \rho^{\vee}(\gamma)\right\}=\left\{\gamma \in \Delta_{+} \mid \gamma \preceq_{c} \beta, \gamma \succeq_{c} \beta\right\}, \\
\mathcal{C}_{+} & =\left\{\gamma \in \Delta_{+} \mid \rho^{\vee}\left(w^{-1} \gamma\right)>t \rho^{\vee}(\gamma)\right\}=\left\{\gamma \in \Delta_{+} \mid \gamma \succ_{c} \beta\right\} .
\end{aligned}
$$

By Lemma 1.23 , the triple $\left(\mathcal{C}_{-}, \mathcal{C}, \mathcal{C}_{+}\right)$is a face. Therefore, $\preceq$ is a convex preorder on $\Delta_{+}$.

We sometimes denote by $\preceq \underline{w}$ a convex order which refines the convex preorder corresponding to $\underline{w}$ given in Proposition 1.24 if we need to emphasize the reduced expression $\underline{w}$.

\section{Categorification of Doubly-Invariant algebras}

2.1. Cuspidal decomposition. In order to recall the notion of cuspidal decomposition, we introduce the following notations. 
Definition 2.1. For $M \in R(\beta)$-Mod, we define

$$
\begin{aligned}
\mathbf{W}(M) & :=\left\{\gamma \in \mathrm{Q}_{+} \cap\left(\beta-\mathrm{Q}_{+}\right) \mid e(\gamma, \beta-\gamma) M \neq 0\right\}, \\
\mathrm{W}^{*}(M) & :=\left\{\gamma \in \mathrm{Q}_{+} \cap\left(\beta-\mathrm{Q}_{+}\right) \mid e(\beta-\gamma, \gamma) M \neq 0\right\} .
\end{aligned}
$$

We have $\mathrm{W}^{*}(M)=\beta-\mathrm{W}(M)$ by the definition. Note that $0, \beta \in \mathrm{W}(M)$ if $M \neq 0$. From the definition, we have the following lemma.

Lemma 2.2. Let $M$ and $N$ be $R$-modules. Then

$$
\mathrm{W}(M \circ N)=\mathrm{W}(M)+\mathrm{W}(N), \quad \mathrm{W}^{*}(M \circ N)=\mathrm{W}^{*}(M)+\mathrm{W}^{*}(N) .
$$

The following proposition is a consequence of [24, Proposition 3.7].

Proposition 2.3. Let $L$ be a simple $R$-module. Then

$\operatorname{span}_{\mathbb{R}_{\geq 0}} \mathrm{~W}(L)=\operatorname{span}_{\mathbb{R}_{\geq 0}}\left(\mathrm{~W}(L) \cap \Delta_{+}\right), \quad \operatorname{span}_{\mathbb{R}_{\geq 0}} \mathrm{~W}^{*}(L)=\operatorname{span}_{\mathbb{R}_{\geq 0}}\left(\mathrm{~W}^{*}(L) \cap \Delta_{+}\right)$.

Let us fix a convex order $\preceq$ on $\Delta_{+}$in this subsection. We set

$$
\begin{aligned}
\Delta_{+}^{\min } & :=\left\{\beta \in \Delta_{+} \mid \mathbb{Q} \beta \cap \Delta_{+} \subset \mathbb{Z}_{>0} \beta\right\}, \\
\mathbb{Z}_{>0} \Delta_{+} & :=\left\{n \gamma \mid n \in \mathbb{Z}_{>0}, \gamma \in \Delta_{+}\right\} \\
& =\left\{n \gamma \mid n \in \mathbb{Z}_{>0}, \gamma \in \Delta_{+}^{\text {min }}\right\} .
\end{aligned}
$$

Note that $\beta \in \mathbb{Z}_{>0} \Delta_{+}$if and only if $\beta \in Q_{+} \cap \operatorname{span}_{\mathbb{R}} \mathcal{C}$ for some $\preceq$-equivalence class $\mathcal{C}$ of $\Delta_{+}$.

We can extend the convex order $\preceq$ on $\Delta_{+}$uniquely to the convex order on $\mathbb{Z}_{>0} \Delta_{+}$ as follows: For $\beta, \beta^{\prime} \in \mathbb{Z}_{>0} \Delta_{+}$, there are $\preceq$-equivalence classes $\mathcal{C}$ and $\mathcal{C}^{\prime}$ such that $\beta \in \operatorname{span}_{\mathbb{R}_{\geq 0}} \mathcal{C}$ and $\beta^{\prime} \in \operatorname{span}_{\mathbb{R}_{\geq 0}} \mathcal{C}^{\prime}$. Then we write $\beta \preceq \beta^{\prime}$ if $\mathcal{C} \preceq \mathcal{C}^{\prime}$.

Note that

for any $\preceq$-equivalence class $\mathcal{C}$ of $\mathbb{Z}_{>0} \Delta_{+}$, there is a unique $\alpha \in \Delta_{+}^{\min }$ such that $\mathcal{C}=\mathbb{Z}_{>0} \alpha$.

Definition 2.4. Let $\beta \in \mathrm{Q}_{+} \backslash\{0\}$. A simple $R(\beta)$-module $L$ is $\preceq$-cuspidal if

(a) $\beta \in \mathbb{Z}_{>0} \Delta_{+}$,

(b) $\mathrm{W}(L) \subset \operatorname{span}_{\mathbb{R}_{>0}}\left\{\gamma \in \Delta_{+} \mid \gamma \preceq \beta\right\}$.

Remark 2.5. The cuspidal modules in our definition are called semi-cuspidal in [24].

By Lemma 1.17, a simple $R(\beta)$-module $L$ is cuspidal if and only if

$$
\beta \in \mathbb{Z}_{>0} \Delta_{+} \text {and } \mathrm{W}^{*}(L) \subset \operatorname{span}_{\mathbb{R}_{\geq 0}}\left\{\gamma \in \Delta_{+} \mid \gamma \succeq \beta\right\} .
$$


Proposition 2.6 ([24, Corollary 2.17]). Let $\beta \in \mathbb{Z}_{>0} \Delta_{+}$. Then, the number of the isomorphism classes of $\preceq$-cuspidal simple $R(\beta)$-modules is

$$
\sum_{\substack{\beta=\gamma_{1}+\cdots+\gamma_{n} \\ \gamma_{k} \in \mathbb{Q} \beta \cap \Delta_{+}}} \prod_{k=1}^{n} m_{\gamma_{k}},
$$

where the sum ranges over all ways of writing $\beta$ as a sum of positive roots in $\mathbb{Q} \beta \cap \Delta_{+}$ (up to permutations). Here, $m_{\gamma}=\operatorname{dim} \mathfrak{g}_{\gamma}$ for $\gamma \in \Delta_{+}$.

Proposition 2.7 ([24, Proposition 2.21]). Let $\beta$ be a real positive root.

(i) For $n \in \mathbb{Z}_{>0}$, there exists a unique self-dual $\preceq$-cuspidal $R(n \beta)$-module $L(n \beta)$ up to an isomorphism.

(ii) For $n \in \mathbb{Z}_{>0}, L(\beta)^{\circ}$ is simple and isomorphic to $L(n \beta)$ up to a grading shift.

Theorem 2.8 ([24, Theorem 2.19]). For a simple $R(\beta)$-module $L$, there exists a unique sequence $\left(L_{1}, L_{2}, \ldots, L_{h}\right)$ of $\preceq$-cuspidal modules (up to isomorphisms) such that

(a) $-\operatorname{wt}\left(L_{k}\right) \succ-\operatorname{wt}\left(L_{k+1}\right)$ for $k=1, \ldots, h-1$,

(b) $L$ is isomorphic to the head of $L_{1} \circ L_{2} \circ \cdots \circ L_{h}$.

The sequence $\left(L_{1}, L_{2}, \ldots, L_{h}\right)$ associated to $L$ given in Theorem 2.8 is called the $\preceq$-cuspidal decomposition of $L$, which is denoted by

$$
\mathfrak{d}_{\preceq}(L):=\left(L_{1}, \ldots, L_{h}\right) .
$$

We write $\mathfrak{d}$ instead of $\mathfrak{d}_{\preceq}$ for simplicity when there is no afraid of confusion. Note that we have

$$
\begin{gathered}
\mathrm{W}(L) \subset \operatorname{span}_{\mathbb{R}_{\geq 0}}\left\{\gamma \in \Delta_{+} \mid \gamma \preceq-\mathrm{wt}\left(L_{1}\right)\right\}, \\
\mathrm{W}^{*}(L) \subset \operatorname{span}_{\mathbb{R}_{\geq 0}}\left\{\gamma \in \Delta_{+} \mid \gamma \succeq-\operatorname{wt}\left(L_{h}\right)\right\} .
\end{gathered}
$$

Definition 2.9. Let $M_{k}$ be $R\left(\beta_{k}\right)$-modules for $k=1, \ldots, n$. We say that $\left(M_{1}, \ldots, M_{n}\right)$ is unmixed if

$$
\operatorname{Res}_{\beta_{1}, \beta_{2}, \ldots, \beta_{n}}\left(M_{1} \circ M_{2} \circ \cdots \circ M_{n}\right)=M_{1} \otimes M_{2} \otimes \cdots \otimes M_{n} .
$$

Then we have the following proposition.

Proposition 2.10 (cf. [24, Proposition 2.6, Lemma 2.8]). Let $M_{k}$ be $R\left(\beta_{k}\right)$-modules for $k=1, \ldots, n$. Suppose that $\left(M_{1}, \ldots, M_{n}\right)$ is unmixed.

(i) For $w \in \mathfrak{S}_{n}$,

$$
\operatorname{Res}_{\beta_{1}, \beta_{2}, \ldots, \beta_{n}}\left(M_{w(1)} \circ M_{w(2)} \circ \cdots \circ M_{w(n)}\right) \simeq q^{t_{w}} M_{1} \otimes M_{2} \otimes \cdots \otimes M_{n},
$$

where $t_{w}=-\sum_{i<j \text { and } w^{-1}(i)>w^{-1}(j)}\left(\beta_{i}, \beta_{j}\right)$. 
(ii) Assume that $M_{j}$ is simple for $1 \leq j \leq n$. Then the head $M$ of $M_{1} \circ M_{2} \circ \cdots \circ M_{n}$ is simple. Moreover, we have

$$
\operatorname{Res}_{\beta_{1}, \beta_{2}, \ldots, \beta_{n}}(M) \simeq M_{1} \otimes M_{2} \otimes \cdots \otimes M_{n} .
$$

Lemma 2.11 ([24, Lemma 2.9]). Let $\left(L_{1}, \ldots, L_{h}\right)$ be a sequence of cuspidal simple $R$ modules with $-\operatorname{wt}\left(L_{1}\right) \succ \cdots \succ-\operatorname{wt}\left(L_{h}\right)$. Then $\left(L_{1}, \ldots, L_{h}\right)$ is unmixed. In particular, for a simple $R$-module $L$, the $\preceq$-cuspidal decomposition $\mathfrak{d}(L)$ is unmixed.

For $m, n \in \mathbb{Z}_{\geq 0}$, we denote by $w[m, n]$ the element of $\mathfrak{S}_{m+n}$ defined by

$$
w[m, n](k)= \begin{cases}k+n & \text { if } 1 \leq k \leq m, \\ k-m & \text { if } m<k \leq m+n .\end{cases}
$$

The following proposition generalizes [7, Proposition 10.1.3].

Proposition 2.12 (cf. [24, Lemma 2.8]). Let $M \in R(\beta)$-mod and $N \in R(\gamma)$-mod, and set $m=\operatorname{ht}(\beta)$ and $n=\operatorname{ht}(\gamma)$. Suppose that $\mathrm{W}^{*}(M) \cap \mathrm{W}(N) \subset\{0\}$. Then we have

(i) $\operatorname{Res}_{\beta, \gamma}(M \circ N)=M \otimes N$ and $\operatorname{Res}_{\beta, \gamma}(N \circ M) \simeq q^{-(\beta, \gamma)} M \otimes N$,

(ii) the map

$$
u \otimes v \mapsto \tau_{w[n, m]}(v \otimes u) \quad \text { for } u \in M, v \in N,
$$

induces an $R(\beta+\gamma)$-linear homomorphism $M \circ N \rightarrow q^{(\beta, \gamma)} N \circ M$.

Proof. For $\beta_{1} \in \mathbf{W}(M)$ and $\gamma_{1} \in \mathbf{W}(N)$ such that $\beta=\beta_{1}+\gamma_{1}$, we have

$$
\beta-\beta_{1}=\gamma_{1} \in \mathbf{W}^{*}(M) \cap \mathbf{W}(N) .
$$

Hence we have $\beta_{1}=\beta$ and $\gamma_{1}=0$. This implies that

$$
e(\beta, \gamma)(M \circ N)=M \otimes N, \quad e(\beta, \gamma)(N \circ M)=\tau_{w[n, m]}(N \otimes M),
$$

which gives (i). Moreover, the map

$$
u \otimes v \mapsto \tau_{w[n, m]}(v \otimes u) \quad \text { for } u \in M, v \in N,
$$

is an $R(\beta) \otimes R(\gamma)$-module homomorphism $M \otimes N \rightarrow q^{(\beta, \gamma)} e(\beta, \gamma)(N \circ M)$. Indeed, for example, for $1 \leq k \leq m$,

$$
\left(x_{k} \tau_{w[n, m]}-\tau_{w[n, m]} x_{k+n}\right)(v \otimes u) \in e(\beta, \gamma)(N \circ M) \cap\left(\sum_{w<w[n, m]} \tau_{w}(N \otimes M)\right)=\{0\},
$$

where the last equality follows from (i).

Lemma 2.13. Let $M_{t}$ be an $R\left(\beta_{t}\right)$-module for $1 \leq t \leq n$ and let $2 \leq k \leq n$. If $\left(M_{1}, \ldots, M_{k-1}\right),\left(M_{k}, \ldots, M_{n}\right)$ and $\left(M_{1} \circ \cdots \circ M_{k-1}, M_{k} \circ \cdots \circ M_{n}\right)$ are unmixed, then $\left(M_{1}, \ldots, M_{n}\right)$ is unmixed. 
Proof. It follows from the transitivity of the induction and restriction.

Lemma 2.14. Let $L$ be a simple $R(\beta)$-module with $\mathfrak{d}(L)=\left(L_{1}, \ldots, L_{h}\right)$. Then

$$
\left(L_{1}, \cdots, L_{k-1},\left(L_{k} \circ \cdots \circ L_{h}\right)\right)
$$

is unmixed for any $2 \leq k \leq h$.

Proof. Note that

$$
\begin{aligned}
& \mathrm{W}^{*}\left(L_{1} \circ \cdots \circ L_{k-1}\right) \cap \mathrm{W}\left(L_{k} \circ \cdots \circ L_{h}\right) \\
& \quad \subset \operatorname{span}_{\mathbb{R}_{\geq 0}}\left\{\gamma \in \Delta_{+} \mid \gamma \succeq-\operatorname{wt}\left(L_{k-1}\right)\right\} \cap \operatorname{span}_{\mathbb{R}_{\geq 0}}\left\{\gamma \in \Delta_{+} \mid \gamma \preceq-\operatorname{wt}\left(L_{k}\right)\right\} \subset\{0\} .
\end{aligned}
$$

By Proposition 2.12, we conclude that $\left(\left(L_{1} \circ \cdots \circ L_{k-1}\right),\left(L_{k} \circ \cdots \circ L_{h}\right)\right)$ is unmixed. Since $\left(L_{1}, \cdots, L_{k-1}\right)$ is unmixed, we get the desired result by Lemma 2.13.

Let $\mathcal{I}_{\beta}$ be the set of sequences $\left(\beta_{1}, \ldots, \beta_{h}\right)$ with $h \geq 1, \beta_{k} \in \mathbb{Z}_{\geq 0} \Delta_{+}$for $1 \leq k \leq h$ and $\beta=\sum_{k=1}^{h} \beta_{k}$.

We define a total order on $\mathcal{I}_{\beta}$ as follows:

$$
\left(\beta_{1}, \beta_{2}, \ldots, \beta_{p}\right) \preceq_{\operatorname{lex}}\left(\beta_{1}^{\prime}, \beta_{2}^{\prime}, \ldots, \beta_{q}^{\prime}\right)
$$

if one of the following conditions holds:

(1) $\left(\beta_{1}, \ldots, \beta_{p}\right)=\left(\beta_{1}^{\prime}, \ldots, \beta_{q}^{\prime}\right)$,

(2) there exists a positive integer $k \leq \min \{p, q\}$ such that

(a) $\beta_{j}=\beta_{j}^{\prime}$ for any $j<k$, and (b) $\beta_{k} \prec \beta_{k}^{\prime}$,

(3) there exists a positive integer $k \leq \min \{p, q\}$ such that

(a) $\beta_{j}=\beta_{j}^{\prime}$ for any $j<k$, (b) $\beta_{k}=t \beta_{k}^{\prime}$ for some $t<1$.

Similarly, we define another total order:

$$
\left(\beta_{1}, \beta_{2}, \ldots, \beta_{p}\right) \preceq_{\text {rev }}\left(\beta_{1}^{\prime}, \beta_{2}^{\prime}, \ldots, \beta_{q}^{\prime}\right)
$$

if one of the following conditions holds:

(1) $\left(\beta_{1}, \ldots, \beta_{p}\right)=\left(\beta_{1}^{\prime}, \ldots, \beta_{q}^{\prime}\right)$,

(2) there exists a positive integer $k \leq \min \{p, q\}$ such that

(a) $\beta_{p-j+1}=\beta_{q-j+1}^{\prime}$ for any $j<k$, and (b) $\beta_{p-k+1} \succ \beta_{q-k+1}^{\prime}$,

(3) there exists a positive integer $k \leq \min \{p, q\}$ such that

(a) $\beta_{p-j+1}=\beta_{q-j+1}^{\prime}$ for any $j<k$, (b) $\beta_{p-k+1}=t \beta_{q-k+1}^{\prime}$ for some $t<1$.

Now we define

$$
\begin{gathered}
\left(\beta_{1}, \beta_{2}, \ldots, \beta_{p}\right) \preceq_{\text {bi }}\left(\beta_{1}^{\prime}, \beta_{2}^{\prime}, \ldots, \beta_{q}^{\prime}\right) \\
\text { if }\left(\beta_{1}, \beta_{2}, \ldots, \beta_{p}\right) \preceq_{\text {lex }}\left(\beta_{1}^{\prime}, \beta_{2}^{\prime}, \ldots, \beta_{q}^{\prime}\right) \text { and }\left(\beta_{1}, \beta_{2}, \ldots, \beta_{p}\right) \preceq_{\text {rev }}\left(\beta_{1}^{\prime}, \beta_{2}^{\prime}, \ldots, \beta_{q}^{\prime}\right) .
\end{gathered}
$$


For a simple $R(\beta)$-module $L$ with $\mathfrak{d}(L)=\left(L_{1}, L_{2}, \ldots, L_{p}\right)$, we set

$$
\mathrm{A}_{\preceq}(L):=\left(-\mathrm{wt}\left(L_{1}\right), \ldots,-\mathrm{wt}\left(L_{p}\right)\right) .
$$

For simple $R(\beta)$-modules, $L$ and $L^{\prime}$ we define

$$
\begin{aligned}
& \mathfrak{d}(L) \preceq_{\text {lex }} \mathfrak{d}\left(L^{\prime}\right) \quad \text { if } \mathrm{A}(L) \preceq_{\text {lex }} \mathrm{A}\left(L^{\prime}\right), \\
& \mathfrak{d}(L) \preceq_{\text {rev }} \mathfrak{d}\left(L^{\prime}\right) \quad \text { if } \mathrm{A}(L) \preceq_{\text {rev }} \mathrm{A}\left(L^{\prime}\right), \text { and } \\
& \mathfrak{d}(L) \preceq_{\text {bi }} \mathfrak{d}\left(L^{\prime}\right) \quad \text { if } \mathrm{A}(L) \preceq_{\text {bi }} \mathrm{A}\left(L^{\prime}\right) .
\end{aligned}
$$

Note that $\preceq_{\text {lex }}$ and $\preceq_{\text {rev }}$ are total preorders on the set of $\preceq$-cuspidal decompositions of all simple $R$-modules.

Proposition 2.15. Let $L$ be a simple $R(\beta)$-module with $\mathfrak{d}(L)=\left(L_{1}, \ldots, L_{h}\right)$ and let $\beta_{k}=-\operatorname{wt}\left(L_{k}\right)$ for $k=1, \ldots, h$.

(i) $L$ appears once in $L_{1} \circ \cdots \circ L_{h}$.

(ii) If $\operatorname{Res}_{\beta_{1}^{\prime}, \ldots, \beta_{t}^{\prime}}\left(L_{1} \circ \cdots \circ L_{h}\right) \neq 0$ for some $\left(\beta_{1}^{\prime}, \ldots, \beta_{t}^{\prime}\right) \in \mathcal{I}_{\beta}$, then we have

$$
\left(\beta_{1}^{\prime}, \ldots, \beta_{t}^{\prime}\right) \preceq_{\text {bi }}\left(\beta_{1}, \ldots, \beta_{h}\right) .
$$

(iii) If $L^{\prime}$ is a simple subquotient of $L_{1} \circ \cdots \circ L_{h}$ which is not isomorphic to $L$, then we have $\mathfrak{d}\left(L^{\prime}\right) \prec_{\mathrm{bi}} \mathfrak{d}(L)$.

Proof. (i) follows from Lemma 2.11.

(ii) Assume that

$$
\left(\beta_{1}, \ldots, \beta_{h}\right) \neq\left(\beta_{1}^{\prime}, \ldots, \beta_{t}^{\prime}\right) .
$$

We shall show that $\left(\beta_{1}^{\prime}, \ldots, \beta_{t}^{\prime}\right) \prec_{\text {lex }}\left(\beta_{1}, \ldots, \beta_{h}\right)$.

Case (l-a) We first consider the case $\beta_{1} \neq \beta_{1}^{\prime}$. Since $\beta_{1}^{\prime} \in \mathbf{W}\left(L_{1} \circ \cdots \circ L_{h}\right)$, Lemma 2.2 allows us to write

$$
\beta_{1}^{\prime}=\gamma_{1}+\gamma_{2} \quad \text { for some } \gamma_{1} \in \mathbf{W}\left(L_{1}\right) \text { and some } \gamma_{2} \in \mathbf{W}\left(L_{2} \circ \cdots \circ L_{h}\right) .
$$

Note that, by Lemma 2.2,

$$
\mathrm{W}\left(L_{2} \circ \cdots \circ L_{h}\right) \subset \operatorname{span}_{\mathbb{R}_{\geq 0}}\left\{\gamma \in \Delta_{+} \mid \gamma \prec \beta_{1}\right\} .
$$

If $\gamma_{2} \neq 0$, then we have $\beta_{1}^{\prime} \prec \beta_{1}$ by Lemma 1.18. Thus we have $\left(\beta_{1}^{\prime}, \ldots, \beta_{t}^{\prime}\right) \preceq_{\text {lex }}$ $\left(\beta_{1}, \ldots, \beta_{h}\right)$.

If $\gamma_{2}=0$, then $\beta_{1}^{\prime} \in \mathrm{W}\left(L_{1}\right)$, which tells $\operatorname{ht}\left(\beta_{1}^{\prime}\right)<\operatorname{ht}\left(\beta_{1}\right)$ since $\beta_{1}^{\prime} \neq \beta_{1}$. If $\beta_{1}^{\prime} \prec \beta_{1}$, then we have $\left(\beta_{1}^{\prime}, \ldots, \beta_{t}^{\prime}\right) \prec_{\text {lex }}\left(\beta_{1}, \ldots, \beta_{h}\right)$. Otherwise, $\beta_{1}$ and $\beta_{1}^{\prime}$ are in the same $\preceq$-equivalence class. Thus, $\beta_{1}^{\prime}=t \beta_{1}$ for some $t<1$, which implies $\left(\beta_{1}^{\prime}, \ldots, \beta_{t}^{\prime}\right) \prec_{\text {lex }}$ $\left(\beta_{1}, \ldots, \beta_{h}\right)$. 
Case (l-b) We now suppose that there is $k \in \mathbb{Z}_{>0}$ such that $\beta_{j}=\beta_{j}^{\prime}$ for any $j<k$ and $\beta_{k} \neq \beta_{k}^{\prime}$. Let $\gamma=\beta-\sum_{j=1}^{k-1} \beta_{j}$. By Lemma 2.14 we have

$$
\operatorname{Res}_{\beta_{1}, \ldots, \beta_{k-1}, \gamma}\left(L_{1} \circ \cdots \circ L_{h}\right) \simeq L_{1} \otimes \cdots \otimes L_{k-1} \otimes\left(L_{k} \circ \cdots \circ L_{h}\right) .
$$

Then the assumption $\operatorname{Res}_{\beta_{1}^{\prime}, \ldots, \beta_{t}^{\prime}}\left(L_{1} \circ \cdots \circ L_{h}\right) \neq 0$ implies

$$
\operatorname{Res}_{\beta_{k}^{\prime}, \ldots, \beta_{h}^{\prime}}\left(L_{k} \circ \cdots \circ L_{h}\right) \neq 0 .
$$

Therefore, by Case (l-a), we conclude that $\left(\beta_{k}^{\prime}, \ldots, \beta_{t}^{\prime}\right) \prec_{\text {lex }}\left(\beta_{k}, \ldots, \beta_{h}\right)$, which yields $\left(\beta_{1}^{\prime}, \ldots, \beta_{t}^{\prime}\right) \prec_{\text {lex }}\left(\beta_{1}, \ldots, \beta_{h}\right)$.

We now shall prove that $\left(\beta_{1}^{\prime}, \ldots, \beta_{t}^{\prime}\right) \prec_{\text {rev }}\left(\beta_{1}, \ldots, \beta_{h}\right)$. The proof is similar to the case of $\prec_{\text {lex }}$.

Case (r-a) Suppose that $\beta_{h} \neq \beta_{t}^{\prime}$. Then, in the same manner as above, we have

$$
\begin{aligned}
\mathrm{W}^{*}\left(L_{h}\right) & \subset \operatorname{span}_{\mathbb{R}_{\geq 0}}\left\{\gamma \in \Delta_{+} \mid \beta_{h} \preceq \gamma\right\}, \\
\mathrm{W}^{*}\left(L_{1} \circ \cdots \circ L_{h-1}\right) & \subset \operatorname{span}_{\mathbb{R}_{\geq 0}}\left\{\gamma \in \Delta_{+} \mid \beta_{h} \prec \gamma\right\} .
\end{aligned}
$$

We write $\beta_{t}^{\prime}=\gamma_{1}+\gamma_{2}$ for some $\gamma_{1} \in \mathbf{W}^{*}\left(L_{h}\right)$ and some $\gamma_{2} \in \mathbf{W}^{*}\left(L_{1} \circ \cdots \circ L_{h-1}\right)$.

If $\gamma_{2} \neq 0$, then $\beta_{h} \prec \beta_{t}^{\prime}$ by Lemma 1.18. Thus we have $\left(\beta_{1}^{\prime}, \ldots, \beta_{t}^{\prime}\right) \prec_{\text {rev }}\left(\beta_{1}, \ldots, \beta_{h}\right)$.

If $\gamma_{2}=0$, then $\beta_{t}^{\prime} \in \mathbf{W}^{*}\left(L_{h}\right)$, which tells $\mathrm{ht}\left(\beta_{t}^{\prime}\right)<\operatorname{ht}\left(\beta_{h}\right)$ since $\beta_{t}^{\prime} \neq \beta_{h}$. If $\beta_{t}^{\prime} \succ \beta_{h}$, then we have $\mathfrak{d}\left(L^{\prime}\right) \prec_{\text {rev }} \mathfrak{d}(L)$. Otherwise, $\beta_{h}$ and $\beta_{t}^{\prime}$ are in the same $\preceq$-equivalence class. Thus, $\beta_{t}^{\prime}=p \beta_{h}$ for some $p<1$, which implies $\left(\beta_{1}^{\prime}, \ldots, \beta_{t}^{\prime}\right) \prec_{\text {rev }}\left(\beta_{1}, \ldots, \beta_{h}\right)$.

Case (r-b) We now assume that there is $k \in \mathbb{Z}_{>0}$ such that $\beta_{h-j+1}=\beta_{t-j+1}^{\prime}$ for any $j<k$ and $\beta_{h-k+1} \neq \beta_{t-k+1}$. Let $\gamma=\beta-\sum_{j=1}^{k-1} \beta_{h-j+1}$. Since

$$
\operatorname{Res}_{\gamma, \beta_{h-k+2}, \ldots, \beta_{h}}\left(L_{1} \circ \cdots \circ L_{h}\right) \simeq\left(L_{1} \circ \cdots \circ L_{h-k+1}\right) \otimes L_{h-k+2} \otimes \cdots \otimes L_{h},
$$

the assumption $\operatorname{Res}_{\beta_{1}^{\prime}, \ldots, \beta_{t}^{\prime}}\left(L_{1} \circ \cdots \circ L_{h}\right) \neq 0$ implies

$$
\operatorname{Res}_{\beta_{1}^{\prime}, \ldots, \beta_{t-k+1}^{\prime}}\left(L_{1} \circ \cdots \circ L_{h-k+1}\right) \neq 0 .
$$

By Case $(\mathbf{r}-\mathbf{a})$, we can conclude that $\left(\beta_{1}^{\prime}, \ldots, \beta_{t-k+1}^{\prime}\right) \prec_{\mathrm{rev}}\left(\beta_{1}, \ldots, \beta_{h-k+1}\right)$, which yields that $\left(\beta_{1}^{\prime}, \ldots, \beta_{t}^{\prime}\right) \prec_{\text {rev }}\left(\beta_{1}, \ldots, \beta_{h}\right)$.

(iii) Let $\mathfrak{d}\left(L^{\prime}\right)=\left(L_{1}^{\prime}, \ldots, L_{t}^{\prime}\right)$ and $\mathrm{A}\left(L^{\prime}\right)=\left(\beta_{1}^{\prime}, \ldots, \beta_{t}^{\prime}\right)$. Since $\operatorname{Res}_{\beta_{1}^{\prime} \ldots, \beta_{t}^{\prime}} L^{\prime} \neq 0$, we have $\operatorname{Res}_{\beta_{1}^{\prime} \ldots, \beta_{t}^{\prime}}\left(L_{1} \circ \cdots \circ L_{h}\right) \neq 0$. It follows from (ii) that $\left(\beta_{1}^{\prime}, \ldots, \beta_{t}^{\prime}\right) \preceq_{\text {bi }}\left(\beta_{1}, \ldots, \beta_{h}\right)$. Note that $\operatorname{Res}_{\beta_{1}, \ldots, \beta_{h}} L^{\prime}=0$ by Theorem 2.8 and hence $\left(\beta_{1}, \ldots, \beta_{h}\right) \neq\left(\beta_{1}^{\prime}, \ldots, \beta_{t}^{\prime}\right)$. Thus we conclude that $\mathfrak{d}\left(L^{\prime}\right) \prec_{\text {bi }} \mathfrak{d}(L)$. 
2.2. Categories $\mathscr{C}_{w, v}$. For $w \in \mathrm{W}$, we denote by $\mathscr{C}_{w}$ the full subcategory of $R$-mod whose objects $M$ satisfy

$$
\mathrm{W}(M) \subset \operatorname{span}_{\mathbb{R}_{\geq 0}}\left(\Delta_{+} \cap w \Delta_{-}\right) .
$$

Similarly, for $v \in \mathrm{W}$, we define $\mathscr{C}_{*, v}$ to be the full subcategory of $R$-mod whose objects $N$ satisfy

$$
\mathrm{W}^{*}(N) \subset \operatorname{span}_{\mathbb{R}_{\geq 0}}\left(\Delta_{+} \cap v \Delta_{+}\right) .
$$

For $M \in \mathscr{C}_{w}$ and $N \in \mathscr{C}_{*, v}$, it is obvious that

$$
\mathrm{W}(M) \subset \mathrm{Q}_{+} \cap w \mathrm{Q}_{-}, \quad \mathrm{W}^{*}(N) \subset \mathrm{Q}_{+} \cap v \mathrm{Q}_{+},
$$

which implies

$$
\mathrm{W}(M) \cap \Delta_{+} \subset \Delta_{+} \cap w \Delta_{-}, \quad \mathrm{W}^{*}(N) \cap \Delta_{+} \subset \Delta_{+} \cap v \Delta_{+} .
$$

By Proposition 2.3, we have the following equivalences:

$$
\begin{aligned}
& M \in \mathscr{C}_{w} \Longleftrightarrow \mathrm{W}(M) \subset \mathrm{Q}_{+} \cap w \mathrm{Q}_{-} \Longleftrightarrow \mathrm{W}(M) \cap \Delta_{+} \subset \Delta_{+} \cap w \Delta_{-}, \\
& N \in \mathscr{C}_{*, v} \Longleftrightarrow \mathrm{W}^{*}(N) \subset \mathrm{Q}_{+} \cap v \mathrm{Q}_{+} \Longleftrightarrow \mathrm{W}^{*}(N) \cap \Delta_{+} \subset \Delta_{+} \cap v \Delta_{+} .
\end{aligned}
$$

For $w, v \in \mathrm{W}$, we define $\mathscr{C}_{w, v}$ to be the full subcategory of $R$-mod whose objects are contained in both of the subcategories $\mathscr{C}_{w}$ and $\mathscr{C}_{*, v}$. By the construction, we have the following proposition.

Proposition 2.16. The categories $\mathscr{C}_{w}, \mathscr{C}_{*, v}$ and $\mathscr{C}_{w, v}$ are stable under taking subquotients, extensions, convolution products and grading shifts. In particular, their Grothendieck groups are $\mathbb{Z}\left[q, q^{-1}\right]$-algebras.

Lemma 2.17. Let $w \in \mathrm{W}$ and $i \in I$.

(i) If $s_{i} w>w$, then $\varepsilon_{i}(L)=0$ for any $L \in \mathscr{C}_{w}$.

(ii) If $s_{i} w<w$, then $\varepsilon_{i}^{*}(L)=0$ for any $L \in \mathscr{C}_{*, w}$.

Proof. (i) Suppose that $L \in \mathscr{C}_{w}$ and $s_{i} w>w$. Then $\alpha_{i} \in \Delta_{+} \cap w \Delta_{+}$, which tells that $\alpha_{i} \notin \mathbf{Q}_{+} \cap w \mathbf{Q}_{-}$. Thus, we have $\alpha_{i} \notin \mathrm{W}(L)$.

(ii) Suppose that $L \in \mathscr{C}_{*, w}$ and $s_{i} w<w$. Then $\alpha_{i} \in \Delta_{+} \cap w \Delta_{-}$, so we have $\alpha_{i} \notin$ $\mathrm{Q}_{+} \cap w \mathbf{Q}_{+}$. This implies $\alpha_{i} \notin \mathbf{W}^{*}(L)$.

The following proposition gives a membership condition on the categories using the cuspidal decomposition. 
Proposition 2.18. Let $\underline{w}=s_{i_{1}} s_{i_{2}} \cdots s_{i_{\ell}}$ be a reduced expression of $w \in \mathrm{W}$. We denote by $\preceq$ a convex order on $\Delta_{+}$which refines the convex preorder with respect to $\underline{w}$ given in Proposition 1.24, and set $\beta_{\ell}=s_{i_{1}} \cdots s_{i_{\ell-1}}\left(\alpha_{i_{\ell}}\right)$. We take a simple $R$-module $L$ and set

$$
\mathfrak{d}(L):=\left(L_{1}, L_{2}, \ldots, L_{h}\right), \quad \gamma_{k}:=-\operatorname{wt}\left(L_{k}\right) \quad \text { for } k=1, \ldots, h .
$$

Then we have

(i) $L \in \mathscr{C}_{w}$ if and only if $\beta_{\ell} \succeq \gamma_{1}$,

(ii) $L \in \mathscr{C}_{*, w}$ if and only if $\gamma_{h} \succ \beta_{\ell}$.

Proof. (i) Suppose that $\beta_{\ell} \succeq \gamma_{1}$. As $\gamma_{1}$ is largest among $\gamma_{k}$ 's, we have

$$
\mathrm{W}\left(L_{1} \circ L_{2} \circ \cdots \circ L_{h}\right) \subset \operatorname{span}_{\mathbb{R}_{\geq 0}}\left(\Delta_{+} \cap w \Delta_{-}\right)
$$

by Lemma 2.2. Thus we have $L \in \mathscr{C}_{w}$.

We assume that $L \in \mathscr{C}_{w}$. By Lemma 2.11, we have

$$
\gamma_{1} \in \mathrm{W}(L) \subset \operatorname{span}_{\mathbb{R}_{\geq 0}}\left(\Delta_{+} \cap w \Delta_{-}\right) .
$$

Since $\beta_{\ell}$ is largest in $\Delta_{+} \cap w \Delta_{-}$, we have $\beta_{\ell} \succeq \gamma_{1}$.

(ii) Suppose that $\gamma_{h} \succ \beta_{\ell}$. Since $\gamma_{h}$ is smallest among $\gamma_{k}$ 's, Lemma 2.2 tells

$$
\mathrm{W}^{*}\left(L_{1} \circ L_{2} \circ \cdots \circ L_{h}\right) \subset \operatorname{span}_{\mathbb{R}_{\geq 0}}\left(\Delta_{+} \cap w \Delta_{+}\right),
$$

which implies $L \in \mathscr{C}_{*, w}$.

We now assume that $L \in \mathscr{C}_{*, w}$. It follows from Lemma 2.11 that

$$
\gamma_{h} \in \mathrm{W}^{*}(L) \subset \operatorname{span}_{\mathbb{R}_{\geq 0}}\left(\Delta_{+} \cap w \Delta_{+}\right) .
$$

Since any positive root in $\Delta_{+} \cap w \Delta_{+}$is larger than $\beta_{\ell}$, we obtain $\gamma_{h} \succ \beta_{\ell}$.

We now revisit the unipotent quantum coordinate ring $A_{q}(\mathfrak{n})_{\mathbb{Z}\left[q, q^{-1}\right]}$. For $w \in \mathbf{W}$, let $A_{w}$ (resp. $A_{*, w}$ ) be the $\mathbb{Z}\left[q, q^{-1}\right]$-linear subspace of $A_{q}(\mathfrak{n})_{\mathbb{Z}\left[q, q^{-1}\right]}$ spanned by all elements $x \in A_{q}(\mathfrak{n})_{\mathbb{Z}\left[q, q^{-1}\right]}$ such that

$$
e_{i_{1}} \cdots e_{i_{l}} x=0 \quad\left(\text { resp. } e_{i_{1}}^{*} \cdots e_{i_{l}}^{*} x=0\right)
$$

for any sequence $\left(i_{1}, \ldots, i_{l}\right) \in I^{\beta}$ with $\beta \in Q_{+} \cap w Q_{+} \backslash\{0\}$ (resp. $\beta \in Q_{+} \cap w Q_{-} \backslash\{0\}$ ).

For $w, v \in \mathbf{W}$, we define

$$
A_{w, v}:=A_{w} \cap A_{*, v} \subset A_{q}(\mathfrak{n})_{\mathbb{Z}\left[q, q^{-1}\right]} .
$$

Remark 2.19. Let $G$ be a reductive algebraic group over $\mathbb{C}$ and $\mathfrak{g}$ its Lie algebra. We fix a maximal torus $H$ of $G$ and take a Borel subgroup $B$ and its opposite Borel subgroup $B^{-}$containing $H$. We denote by $N$ and $N^{-}$the unipotent radicals of $B$ and $B^{-}$respectively, and $\mathfrak{n}$ and $\mathfrak{n}^{-}$denote the Lie algebras of $N$ and $N^{-}$. Let $\mathbf{W}:=$ 
$\operatorname{Norm}_{G}(H) / H$ be the Weyl group. Let $\mathbb{C}[N]$ be the coordinate ring of $N$ and, for $w, v \in \mathrm{W}$,

$$
N^{\prime}(w):=N \cap\left(w N w^{-1}\right) \quad \text { and } \quad N(v):=N \cap\left(v N^{-} v^{-1}\right) .
$$

Note that $\mathbb{C}[N]$ is isomorphic to the dual of $U(\mathfrak{n})$. We consider the doubly-invariant algebra

$$
N^{\prime}(w) \mathbb{C}[N]^{N(v)}:=\left\{f \in \mathbb{C}[N] \mid f(n x m)=f(x) \text { for all } x \in N, m \in N^{\prime}(w), n \in N(v)\right\}
$$

which was introduced in [17]. Then we have

$$
{ }^{N^{\prime}(w)} \mathbb{C}[N]^{N(v)}=\left\{f \in \mathbb{C}[N] \mid x f y=0 \text { for all } x \in \mathfrak{n} \cap w \mathfrak{n}, y \in \mathfrak{n} \cap v \mathfrak{n}^{-}\right\} .
$$

By the PBW theorem, we have

$$
U(\mathfrak{n})=U\left(\mathfrak{n} \cap u \mathfrak{n}^{-}\right) U(\mathfrak{n} \cap u \mathfrak{n}) \quad \text { for any } u \in \mathbf{W} .
$$

Hence, we have

$$
\begin{aligned}
\sum_{\beta \in \mathbf{Q}_{+} \cap w \mathbf{Q}_{+} \backslash\{0\}} U(\mathfrak{n})_{\beta} & \subset \sum_{\substack{\beta \in \mathbf{Q}_{+} \cap w \mathbf{Q}_{+} \backslash\{0\}, \gamma \in w \mathbf{Q}_{-}}} U\left(\mathfrak{n} \cap w \mathfrak{n}^{-}\right)_{\gamma} U(\mathfrak{n} \cap w \mathfrak{n})_{\beta-\gamma} \\
\subset & \sum_{\alpha \in Q_{+} \cap w \mathbf{Q}_{+} \backslash\{0\}} U\left(\mathfrak{n} \cap w \mathfrak{n}^{-}\right) U(\mathfrak{n} \cap w \mathfrak{n})_{\alpha} .
\end{aligned}
$$

Similarly, we have

$$
\begin{aligned}
\sum_{\beta \in Q_{+} \cap v \mathbf{Q}_{-} \backslash\{0\}} U(\mathfrak{n})_{\beta} & \subset \sum_{\substack{\beta \in Q_{+} \cap v \mathbf{Q}_{-} \backslash\{0\}, \gamma \in v \mathbf{Q}_{+}}} U\left(\mathfrak{n} \cap v \mathfrak{n}^{-}\right)_{\beta-\gamma} U(\mathfrak{n} \cap v \mathfrak{n})_{\gamma} \\
& \subset \sum_{\alpha \in Q_{+} \cap v \mathbf{Q}_{-} \backslash\{0\}} U\left(\mathfrak{n} \cap v \mathfrak{n}^{-}\right)_{\alpha} U(\mathfrak{n} \cap v \mathfrak{n}) .
\end{aligned}
$$

Thus, for $f \in N^{\prime}(w) \mathbb{C}[N]^{N(v)}, \beta \in \mathrm{Q}_{+} \cap w \mathrm{Q}_{+} \backslash\{0\}$ and $\gamma \in \mathrm{Q}_{+} \cap v \mathrm{Q}_{-} \backslash\{0\}$, we have

$$
U(\mathfrak{n})_{\beta} f U(\mathfrak{n})_{\gamma}=0
$$

which implies that

$$
\begin{aligned}
& N^{\prime}(w) \mathbb{C}[N]^{N(v)}=\left\{f \in \mathbb{C}[N] \mid U(\mathfrak{n})_{\beta} f U(\mathfrak{n})_{\gamma}=0\right. \\
& \text { for any } \left.\beta \in Q_{+} \cap w Q_{+} \backslash\{0\} \text { and } \gamma \in Q_{+} \cap v Q_{-} \backslash\{0\}\right\} \text {. }
\end{aligned}
$$

This tells that the ring ${ }^{N^{\prime}(w)} \mathbb{C}[N]^{N(v)}$ is the subspace of $\mathbb{C}[N]$ consisting of all elements $f$ such that

$$
e_{i_{1}} \cdots e_{i_{l}} f=0 \quad \text { and } \quad e_{j_{1}}^{*} \cdots e_{j_{t}}^{*} f=0
$$


for all $\left(i_{1}, \ldots, i_{l}\right) \in I^{\beta}$ with $\beta \in Q_{+} \cap w Q_{+} \backslash\{0\}$ and all $\left(j_{1}, \ldots, j_{t}\right) \in I^{\gamma}$ with $\gamma \in$ $\mathrm{Q}_{+} \cap v \mathrm{Q}_{-} \backslash\{0\}$. Therefore, $A_{w, v}$ can be thought of as a quantum deformation of $N^{\prime}(w) \mathbb{C}[N]^{N(v)}$.

Theorem 2.20. Let $w, v \in \mathrm{W}$.

(i) Let $x \in A_{q}(\mathfrak{n})_{\mathbb{Z}\left[q, q^{-1}\right]}$.

(1) The following conditions are equivalent:

(a) $e_{i_{1}} \cdots e_{i_{l}} x=0$ for any $\left(i_{1}, \ldots, i_{l}\right) \in I^{\beta}$ with $\beta \in Q_{+} \backslash w Q_{-}$,

(b) $e_{i_{1}} \cdots e_{i_{l}} x=0$ for any $\left(i_{1}, \ldots, i_{l}\right) \in I^{\beta}$ with $\beta \in Q_{+} \cap w Q_{+} \backslash\{0\}$,

(c) $e_{i_{1}} \cdots e_{i_{l}} x=0$ for any $\left(i_{1}, \ldots, i_{l}\right) \in I^{\beta}$ with $\beta \in \Delta_{+} \cap w \Delta_{+}$.

(2) The following conditions are equivalent:

(a) $e_{i_{1}}^{*} \cdots e_{i_{l}}^{*} x=0$ for any $\left(i_{1}, \ldots, i_{l}\right) \in I^{\beta}$ with $\beta \in Q_{+} \backslash v Q_{+}$,

(b) $e_{i_{1}}^{*} \cdots e_{i_{l}}^{*} x=0$ for any $\left(i_{1}, \ldots, i_{l}\right) \in I^{\beta}$ with $\beta \in Q_{+} \cap v Q_{-} \backslash\{0\}$,

(c) $e_{i_{1}}^{*} \cdots e_{i_{1}}^{*} x=0$ for any $\left(i_{1}, \ldots, i_{l}\right) \in I^{\beta}$ with $\beta \in \Delta_{+} \cap v \Delta_{-}$.

(ii) Under the identification via the isomorphism $K_{0}(R$-mod $) \simeq A_{q}(\mathfrak{n})_{\mathbb{Z}\left[q, q^{-1}\right]}$, we have

(a) $K_{0}\left(\mathscr{C}_{w}\right)=A_{w}$,

(b) $K_{0}\left(\mathscr{C}_{*, v}\right)=A_{*, v}$,

(c) $K_{0}\left(\mathscr{C}_{w, v}\right)=A_{w, v}$.

Proof. We first deal with the case (i) (1) and (ii) (a) together.

$$
\begin{aligned}
& A:=\left\{x \in A_{q}(\mathfrak{n})_{\mathbb{Z}\left[q, q^{-1}\right]} \mid e_{i_{1}} \cdots e_{i_{l}} x=0 \text { for any }\left(i_{1}, \ldots, i_{l}\right) \in I^{\gamma} \text { with } \gamma \in Q_{+} \backslash w Q_{-}\right\}, \\
& B:=\left\{x \in A_{q}(\mathfrak{n})_{\mathbb{Z}\left[q, q^{-1}\right]} \mid e_{i_{1}} \cdots e_{i_{l}} x=0 \text { for any }\left(i_{1}, \ldots, i_{l}\right) \in I^{\gamma} \text { with } \gamma \in Q_{+} \cap w Q_{+}\right\}, \\
& C:=\left\{x \in A_{q}(\mathfrak{n})_{\mathbb{Z}\left[q, q^{-1}\right]} \mid e_{i_{1}} \cdots e_{i_{l}} x=0 \text { for any }\left(i_{1}, \ldots, i_{l}\right) \in I^{\gamma} \text { with } \gamma \in \Delta_{+} \cap w \Delta_{+}\right\}, \\
& K:=K_{0}\left(\mathscr{C}_{w}\right) .
\end{aligned}
$$

We shall show that all of four spaces coincide. By the definition, we have

$$
A \subset B \subset C .
$$

Thus it suffices to show that $K \subset A$ and $C \subset K$.

Inclusion $K \subset A$ : Let $M$ be a simple $R(\beta)$-module in $\mathscr{C}_{w}$. We take a sequence $\left(i_{1}, \ldots, i_{l}\right) \in I^{\gamma}$ with $\gamma \in Q_{+} \backslash w Q_{-}$. By $(2.5)$, we have

$$
\operatorname{Res}_{\gamma, \beta-\gamma} M=0
$$

which implies that $e_{i_{1}} \cdots e_{i_{l}}[M]=0$. Thus, we have $[M] \in A$.

Inclusion $C \subset K$ : Let $\preceq$ be a convex order on $\Delta_{+}$which refines the convex preorder corresponding to a reduced expression of $w$ given in Proposition 1.24. 
Let $f$ be an element in $C$. We shall show that $f \in K$. By Theorem 1.9, $f$ can be written as

$$
f=\sum_{k=1}^{m} a_{k}\left[M_{k}\right]
$$

for some non-zero $a_{k} \in \mathbb{Z}\left[q, q^{-1}\right]$ and some self-dual simple $R$-modules $M_{k}$ which are not isomorphic to each other. We may assume $m>0$. Without loss of generality, we may assume that

$$
\mathfrak{d}\left(M_{j}\right) \succeq_{\text {lex }} \mathfrak{d}\left(M_{j+1}\right) \quad \text { for } 1 \leq j<m .
$$

We write $\mathfrak{d}\left(M_{1}\right)=\left(L_{1}, L_{2}, \ldots, L_{h}\right)$ and let $\gamma_{k}=-\operatorname{wt}\left(L_{k}\right)$ for $k=1, \ldots, h$. Note that

$$
\mathrm{W}\left(M_{j}\right) \subset \operatorname{span}_{\mathbb{R}_{\geq 0}}\left\{x \in \Delta_{+} \mid \gamma_{1} \succeq x\right\} \quad \text { for } j=1, \ldots, m .
$$

It follows from Lemma 2.11 and Proposition 2.15 that, for $j=1, \ldots, m$,

$$
\operatorname{Res}_{\gamma_{1}, \ldots, \gamma_{h}}\left(M_{j}\right) \simeq \begin{cases}0 & \text { if } \mathfrak{d}\left(M_{j}\right) \prec_{\text {lex }} \mathfrak{d}\left(M_{1}\right), \\ L_{j, 1} \otimes \cdots \otimes L_{j, h_{j}} & \text { otherwise }\end{cases}
$$

where $\mathfrak{d}\left(M_{j}\right)=\left(L_{j, 1}, \ldots, L_{j, h_{j}}\right)$.

By (2.2), we can write $\gamma_{1}=n \alpha$ for some $n \in \mathbb{Z}_{>0}$ and some $\alpha \in \Delta_{+}^{\min }$.

(a) Suppose that $\alpha \in \Delta_{+} \cap w \Delta_{-}$. By (2.6) and the definition of the convex order $\preceq$, we have

$$
\mathrm{W}\left(M_{j}\right) \subset \operatorname{span}_{\mathbb{R}_{\geq 0}}\left\{\beta^{\prime} \in \Delta_{+} \mid \beta^{\prime} \preceq \alpha\right\} \subset \mathrm{Q}_{+} \cap w \mathrm{Q}_{-} \quad \text { for } j=1, \ldots, m,
$$

which implies $f \in K$.

(b) Suppose that $\alpha \in \Delta_{+} \cap w \Delta_{+}$.

(b1) Assume first that $\alpha$ is an imaginary root. Then, $\gamma_{1}=c \alpha \in \Delta_{+} \cap w \Delta_{+}$. Letting $d=\operatorname{ht}\left(\gamma_{1}\right)$, we have

$$
e_{i_{1}} \cdots e_{i_{d}} f=0 \text { for any sequence }\left(i_{1}, \ldots, i_{d}\right) \in I^{\gamma_{1}},
$$

which implies

$$
0=\sum_{j=1}^{m} a_{j} \operatorname{ch}_{q}\left(\operatorname{Res}_{\gamma_{1}, \ldots, \gamma_{h}} M_{j}\right) .
$$

By (2.7), $\operatorname{Res}_{\gamma_{1}, \ldots, \gamma_{h}}\left(M_{j}\right)$ is zero or is isomorphic to an outer tensor product of simple modules. Thus the set

$$
\left\{\operatorname{ch}_{q}\left(\operatorname{Res}_{\gamma_{1}, \ldots, \gamma_{h}}\left(M_{j}\right)\right) \mid \operatorname{Res}_{\gamma_{1}, \ldots, \gamma_{h}} M_{j} \neq 0, j=1, \ldots, m\right\}
$$

is linearly independent. It follows from $\operatorname{Res}_{\gamma_{1}, \ldots, \gamma_{h}}\left(M_{1}\right) \neq 0$ that

$$
a_{1}=0,
$$


which is a contradiction to the non-vanishing assumption on $a_{1}$. Therefore we conclude that $f \in K$.

(b2) Assume now that $\alpha$ is a real root. We set $\mu=-\operatorname{wt}(f)=-\operatorname{wt}\left(M_{j}\right) \in \mathrm{Q}_{+}$. We set $\beta_{j}=-\operatorname{wt}\left(L_{j, 1}\right)$ where we set $\mathfrak{d}\left(M_{j}\right)=\left(L_{j, 1}, \ldots, L_{j, h_{j}}\right)$. We set $\left\{j \mid \beta_{j}=\beta_{1}=n \alpha\right\}=$ $[1, b]$ with $1 \leq b \leq m$. Then for $j>b$ we have either $\beta_{j}=m \alpha$ for some $m<n$ or $\beta_{j} \prec \alpha$.

Then by Proposition 2.15, we have

$$
\operatorname{Res}_{n \alpha, \mu-n \alpha}\left(M_{j}\right) \simeq \begin{cases}L_{j, 1} \otimes \operatorname{hd}\left(L_{j, 2} \circ \cdots \circ L_{j, h_{j}}\right) & \text { if } j \in[1, b] \\ 0 & \text { otherwise. }\end{cases}
$$

By Proposition 2.7, we have $L_{j, 1} \simeq L(n \alpha)$ for $j \in[1, b]$. Here $L(\alpha)$ is the $\preceq$-cuspidal simple $R(\alpha)$-module and $L(n \alpha) \simeq L(\alpha)^{\circ n}$ up to grading shift. Let us take $\left(i_{1}, \ldots, i_{d}\right) \in$ $I^{\alpha}$ such that $E_{i_{1}} \cdots E_{i_{d}} L(\alpha)$ does not vanish. Then we have

$$
\left[\left(E_{i_{1}} \cdots E_{i_{d}}\right)^{n} L(n \alpha)\right]=c
$$

for some $c \in \mathbb{Z}_{\geq 0}\left[q, q^{-1}\right] \backslash\{0\}$. Thus we obtain

$$
\left(e_{i_{1}} \cdots e_{i_{d}}\right)^{n} f=\sum_{j=1}^{m} a_{j}\left[\left(E_{i_{1}} \cdots E_{i_{d}}\right)^{n} M_{j}\right]=c \sum_{j=1}^{b} a_{j}\left[\operatorname{hd}\left(L_{j, 2} \circ \cdots \circ L_{j, h_{j}}\right)\right] .
$$

Since $\left\{\left[\operatorname{hd}\left(L_{j, 2} \circ \cdots \circ L_{j, h_{j}}\right)\right]\right\}_{j \in[1, b]}$ is linearly independent, it contradicts $\left(e_{i_{1}} \cdots e_{i_{d}}\right) f=$ 0 .

Thus we obtain $A=B=C=K$.

In a similar manner as above, one can prove (i) (2) and (ii) (b).

Assertion (ii) (c) follows immediately from (ii) (a) and (ii) (b).

Remark 2.21. (i) Since $A_{q}(\mathfrak{n})$ is the dual of $U_{q}^{+}(\mathfrak{g})$, Theorem 2.20 (i) implies that

$$
\sum_{\beta \in Q_{+} \backslash w \mathbf{Q}_{\mp}} U_{q}^{+}(\mathfrak{g}) U_{q}^{+}(\mathfrak{g})_{\beta}=\sum_{\beta \in Q_{+} \cap w \mathbf{Q}_{ \pm} \backslash\{0\}} U_{q}^{+}(\mathfrak{g}) U_{q}^{+}(\mathfrak{g})_{\beta}=\sum_{\beta \in \Delta_{+} \cap w \Delta_{ \pm}} U_{q}^{+}(\mathfrak{g}) U_{q}^{+}(\mathfrak{g})_{\beta} .
$$

(ii) By specializing at $q=1$, the same properties as in Theorem 2.20 (i) hold for $\mathbb{C}[N]^{N(v)}, N^{\prime}(w) \mathbb{C}[N]$ and ${ }^{N^{\prime}(w)} \mathbb{C}[N]^{N(v)}$. Indeed, the same proof of Theorem 2.20 can be applied by replacing the categories of graded modules over quiver Hecke algebras with their non-graded versions. The constant $c$ in (2.8) becomes then a positive integer. Note that the analogue of (2.9) at $q=1$ still holds.

Corollary 2.22. The subspaces $A_{w}, A_{*, v}$ and $A_{w, v}$ are subalgebras of $A_{q}(\mathfrak{n})_{\mathbb{Z}\left[q, q^{-1}\right]}$.

Proof. It follows from Proposition 2.16 and Theorem 2.20. 
Remark 2.23. Suppose that $A$ is of finite type. Then there is the longest element $w_{0} \in \mathbf{W}$ and

$$
\mathrm{Q}_{+} \cap w \mathrm{Q}_{-}=\mathrm{Q}_{+} \cap w w_{0} \mathrm{Q}_{+}, \quad \mathrm{Q}_{+} \cap v \mathrm{Q}_{+}=\mathrm{Q}_{+} \cap v w_{0} \mathrm{Q}_{-}
$$

for $w, v \in \mathrm{W}$. Thus, the algebra involution $*$ of $R(\beta)$ gives an equivalence of two categories $\mathscr{C}_{w, v}$ and $\mathscr{C}_{v w_{0}, w w_{0}}$, where $*: R(\beta) \rightarrow R(\beta)$ is given by

$$
e(\nu) \mapsto e(\bar{\nu}), \quad x_{k} \mapsto x_{n-k+1}, \quad \tau_{l} \mapsto-\tau_{n-l} .
$$

Here $n=\operatorname{ht}(\beta)$ and $\bar{\nu}$ denotes the reversed sequence $\left(\nu_{n}, \nu_{n-1}, \ldots, \nu_{2}, \nu_{1}\right)$ of $\nu=$ $\left(\nu_{1}, \ldots, \nu_{n}\right)$.

\section{R-MATRICES}

3.1. Strongly commuting pairs. In this subsection, we assume that the quiver Hecke algebra $R$ is arbitrary.

For simple $R$-modules $M$ and $N$, we say that $M$ and $N$ strongly commute or $M$ strongly commutes with $N$ if $M \circ N$ is simple. We say that a simple $R$-module $L$ is real if $L$ strongly commutes with itself, i.e., if $L \circ L$ is simple.

For $M \in R(\beta)$-mod, we define $M^{\star}:=\operatorname{Hom}_{\mathbf{k}}(M, \mathbf{k})$, which admits an $R(\beta)$-module structure via

$$
(r \cdot f)(u):=f(\psi(r) u) \quad \text { for } f \in M^{\star}, r \in R(\beta) \text { and } u \in M,
$$

where $\psi$ denotes the anti-automorphism of $R(\beta)$ which fixes the generators. We say that a simple module $L$ is self-dual if $L \simeq L^{\star}$. It is known that

- for a simple $R$-module $L$, there exists $n \in \mathbb{Z}$ such that $q^{n} L$ is self-dual,

- for $M, N \in R$-mod, $N^{\star} \circ M^{\star} \simeq q^{-(\operatorname{wt}(M), \operatorname{wt}(N))}(M \circ N)^{\star}$.

Thus, if two simple modules $M$ and $N$ strongly commute, then we have

$$
M \circ N \simeq N \circ M
$$

up to a grading shift.

Lemma 3.1. Let $M$ and $N$ be simple $R$-modules. Suppose that $M$ and $N$ strongly commute. Then we have

(i) $\widetilde{E}_{i}^{\max } M$ and $\widetilde{E}_{i}^{\max } N$ strongly commute,

(ii) $\widetilde{E}_{i}^{* \max } M$ and $\widetilde{E}_{i}^{* \max } N$ strongly commute.

Proof. (i) Letting $m=\varepsilon_{i}(M)$ and $n=\varepsilon_{i}(N)$, we get $E_{i}^{(m+n)}(M \circ N) \simeq E_{i}^{(m)} M \circ E_{i}^{(n)} N$ up to a grading shift and it is simple. 
(ii) can be proved in the same manner as above by replacing the role of $E_{i}$ with the one of $E_{i}^{*}$.

Lemma 3.2. Let $M$ and $N$ be simple $R$-modules and $i \in I$.

(i) If $M$ strongly commutes with $L(i)$, then $\varepsilon_{i}(M \nabla N)=\varepsilon_{i}(M)+\varepsilon_{i}(N)$.

(ii) If $N$ strongly commutes with $L(i)$, then $\varepsilon_{i}^{*}(M \nabla N)=\varepsilon_{i}^{*}(M)+\varepsilon_{i}^{*}(N)$.

Proof. (i) We set $M_{0}:=E_{i}^{(m)} M$ and $N_{0}:=E_{i}^{(n)} N$, where $m=\varepsilon_{i}(M)$ and $n=\varepsilon_{i}(N)$. Then we have

$$
L\left(i^{m+n}\right) \circ M_{0} \circ N_{0} \rightarrow L\left(i^{n}\right) \circ M \circ N_{0} \simeq M \circ L\left(i^{n}\right) \circ N_{0} \rightarrow M \circ N \rightarrow M \nabla N,
$$

up to grading shifts, which implies that $\varepsilon_{i}(M \nabla N)=m+n$.

(ii) can be proved in the same manner as (i).

3.2. Normalized R-matrices. We recall the notions of affinizations and $R$-matrices for symmetric quiver Hecke algebras introduced in [5]. Note that they have been generalized to arbitrary quiver Hecke algebras in [10].

Let $\beta \in \mathrm{Q}_{+}$and $m=\operatorname{ht}(\beta)$. For $k=1, \ldots, m-1$ and $\nu \in I^{\beta}$, the intertwiner $\varphi_{k} \in R(\beta)$ is defined by

$$
\varphi_{k} e(\nu):= \begin{cases}\left(\tau_{k} x_{k}-x_{k} \tau_{k}\right) e(\nu) & \text { if } \nu_{k}=\nu_{k+1} \\ \tau_{k} e(\nu) & \text { otherwise. }\end{cases}
$$

Then $\left\{\varphi_{k}\right\}_{1 \leq k \leq m-1}$ satisfies the braid relation. Hence, for $w \in \mathfrak{S}_{m}$, the element $\varphi_{w}:=$ $\varphi_{i_{1}} \cdots \varphi_{i_{\ell}}$ does not depend on the choice of a reduced expression $w=s_{i_{1}} \cdots s_{i_{\ell}}$ of $w$.

Let $M$ be an $R(\beta)$-module with ht $(\beta)=m$ and $N$ an $R\left(\beta^{\prime}\right)$-module with ht $\left(\beta^{\prime}\right)=n$. Then the $R(\beta) \otimes R\left(\beta^{\prime}\right)$-linear map $M \otimes N \longrightarrow N \circ M$ defined by $u \otimes v \mapsto \varphi_{w[n, m]}(v \otimes u)$ can be extended to the $R\left(\beta+\beta^{\prime}\right)$-module homomorphism (up to a grading shift)

$$
R_{M, N}: M \circ N \longrightarrow N \circ M \text {. }
$$

Definition 3.3. The quiver Hecke algebra $R(\beta)$ is said to be symmetric if $\mathcal{Q}_{i, j}(u, v)$ is a polynomial in $u-v$ for any $i, j \in I$.

We now assume that $R(\beta)$ is symmetric. Then the generalized Cartan matrix $\mathrm{A}$ is symmetric. In this case we assume

$$
\left(\alpha_{i}, \alpha_{i}\right)=2 \text { for any } i \in I \text {. }
$$

Let $z$ be an indeterminate. For an $R(\beta)$-module $M$, the $R(\beta)$-module structure on the affinization $M_{z}:=\mathbf{k}[z] \otimes_{\mathbf{k}} M$ of $M$ is defined by

$e(\nu)(f \otimes m)=f \otimes e(\nu) m, x_{j}(f \otimes m)=(z f) \otimes m+f \otimes x_{j} m, \tau_{k}(f \otimes m)=f \otimes\left(\tau_{k} m\right)$ 
for $f \in \mathbf{k}[z], m \in M, \nu \in I^{\beta}$ and admissible $j, k$. For non-zero $R$-modules $M$ and $N$, we set

$$
R_{M_{z}, N_{z^{\prime}}}^{\text {norm }}:=\left(z^{\prime}-z\right)^{-s} R_{M_{z}, N_{z^{\prime}}}: M_{z} \circ N_{z^{\prime}} \rightarrow N_{z^{\prime}} \circ M_{z},
$$

where $s$ is the largest integer such that $R_{M_{z}, N_{z^{\prime}}}\left(M_{z} \circ N_{z^{\prime}}\right) \subset\left(z^{\prime}-z\right)^{s} N_{z^{\prime}} \circ M_{z}$. We call it the normalized $R$-matrix.

Then, we have the intertwiner (up to a grading shift)

$$
\mathbf{r}_{M, N}: M \circ N \rightarrow N \circ M
$$

induced from $R_{M_{z}, N_{z^{\prime}}}^{\text {norm }}$ by specializing at $z=z^{\prime}=0$, which never vanishes by the definition.

Definition 3.4. Let $M$ and $N$ be simple $R$-modules. We set

$$
\begin{aligned}
\Lambda(M, N) & :=\operatorname{deg}\left(\mathbf{r}_{M, N}\right), \\
\widetilde{\Lambda}(M, N) & :=\frac{1}{2}(\Lambda(M, N)+(\operatorname{wt}(M), \operatorname{wt}(N))), \\
\mathfrak{D}(M, N) & :=\frac{1}{2}(\Lambda(M, N)+\Lambda(N, M)) .
\end{aligned}
$$

Proposition 3.5 ([6]). Let $M$ and $N$ be simple $R$-modules. Assume that one of $M$ and $N$ is real.

(i) $M \circ N$ has a simple head and a simple socle. Moreover, $\operatorname{Im}\left(\mathbf{r}_{M, N}\right)$ is equal to $M \nabla N$ and $N \Delta M$ up to grading shifts.

(ii) We have

$$
\operatorname{Hom}_{R}(M \circ N, M \circ N)=\operatorname{kid}_{M \circ N}, \quad \operatorname{Hom}_{R}(M \circ N, N \circ M)=\mathbf{k r}_{M, N} .
$$

(iii) If $M \nabla N$ and $M \Delta N$ are isomorphic, then $M$ and $N$ strongly commute.

(iv) $M$ and $N$ strongly commute if and only if $\mathfrak{b}(M, N)=0$.

Lemma 3.6. Let $M$ and $N$ be simple $R$-modules and let $i \in I$. Suppose

(a) one of $M$ and $N$ is real,

(b) $M$ and $N$ strongly commute with $L(i)$.

Then we have the following.

(i) If $\widetilde{E}_{i}^{\max } M$ and $\widetilde{E}_{i}^{\max } N$ strongly commute, then $M$ and $N$ strongly commute.

(ii) If $\widetilde{E}_{i}^{* \max } M$ and $\widetilde{E}_{i}^{* \max } N$ strongly commute, then $M$ and $N$ strongly commute.

Proof. (i) We set

$$
m=\varepsilon_{i} M, \quad n=\varepsilon_{i} N, \quad M_{0}=\widetilde{E}_{i}^{\max } M, \quad N_{0}=\widetilde{E}_{i}^{\max } N .
$$


By the assumption, $M_{0} \circ N_{0}$ is simple which tells that $L\left(i^{m+n}\right) \circ M_{0} \circ N_{0}$ has a simple head. Since $M$ and $L(i)$ strongly commute, we have

$$
\begin{aligned}
\operatorname{hd}\left(L\left(i^{m+n}\right) \circ M_{0} \circ N_{0}\right) & \simeq \operatorname{hd}\left(L\left(i^{n}\right) \circ L\left(i^{m}\right) \circ M_{0} \circ N_{0}\right) \simeq \operatorname{hd}\left(L\left(i^{n}\right) \circ M \circ N_{0}\right) \\
& \simeq \operatorname{hd}\left(M \circ L\left(i^{n}\right) \circ N_{0}\right) \simeq \operatorname{hd}(M \circ N) \simeq M \nabla N,
\end{aligned}
$$

up to grading shifts. In the same manner, we have

$$
\operatorname{hd}\left(L\left(i^{m+n}\right) \circ N_{0} \circ M_{0}\right) \simeq N \nabla M
$$

up to grading shifts. Thus it follows from $M_{0} \circ N_{0} \simeq N_{0} \circ M_{0}$ that $M \nabla N \simeq N \nabla M$, which yields the assertion by Proposition 3.5.

(ii) can be proved in the same manner as above by replacing the role of $E_{i}$ with the one of $E_{i}^{*}$.

Proposition 3.7. Let $M$ and $N$ be simple R-modules. Suppose that one of $M$ and $N$ is real.

(i) Let $m=\varepsilon_{i}(M)$ and $n=\varepsilon_{i}(N)$. If $\varepsilon_{i}(M \nabla N)=m+n$, then

$$
\begin{aligned}
\Lambda(M, N) & =\Lambda\left(M_{0}, N_{0}\right)-n\left(\alpha_{i}, \operatorname{wt}\left(M_{0}\right)\right)+m\left(\alpha_{i}, \operatorname{wt}\left(N_{0}\right)\right) \\
& =\Lambda\left(M_{0}, N_{0}\right)-n\left(\alpha_{i}, \operatorname{wt}(M)\right)+m\left(\alpha_{i}, \operatorname{wt}(N)\right),
\end{aligned}
$$

where $M_{0}=E_{i}^{(m)} M$ and $N_{0}=E_{i}^{(n)} N$.

(ii) Let $m^{\prime}=\varepsilon_{i}^{*}(M)$ and $n^{\prime}=\varepsilon_{i}^{*}(N)$. If $\varepsilon_{i}^{*}(M \nabla N)=m^{\prime}+n^{\prime}$, then

$$
\begin{aligned}
\Lambda(M, N) & =\Lambda\left(M_{0}^{\prime}, N_{0}^{\prime}\right)+n^{\prime}\left(\alpha_{i}, \operatorname{wt}\left(M_{0}^{\prime}\right)\right)-m^{\prime}\left(\alpha_{i}, \operatorname{wt}\left(N_{0}^{\prime}\right)\right) \\
& =\Lambda\left(M_{0}^{\prime}, N_{0}^{\prime}\right)+n^{\prime}\left(\alpha_{i}, \operatorname{wt}(M)\right)-m^{\prime}\left(\alpha_{i}, \operatorname{wt}(N)\right),
\end{aligned}
$$

where $M_{0}^{\prime}=E_{i}^{*\left(m^{\prime}\right)} M$ and $N_{0}^{\prime}=E_{i}^{*\left(n^{\prime}\right)} N$.

Proof. Note that

$$
\begin{aligned}
m\left(\alpha_{i}, \operatorname{wt}\left(N_{0}\right)\right)-n\left(\alpha_{i}, \operatorname{wt}\left(M_{0}\right)\right) & =m\left(\alpha_{i}, \operatorname{wt}(N)\right)-n\left(\alpha_{i}, \operatorname{wt}(M)\right), \\
m^{\prime}\left(\alpha_{i}, \operatorname{wt}\left(N_{0}^{\prime}\right)\right)-n^{\prime}\left(\alpha_{i}, \operatorname{wt}\left(M_{0}^{\prime}\right)\right) & =m^{\prime}\left(\alpha_{i}, \operatorname{wt}(N)\right)-n^{\prime}\left(\alpha_{i}, \operatorname{wt}(M)\right) .
\end{aligned}
$$

(i) By [7, Proposition 10.1.5], we have

$$
\begin{aligned}
& E_{i}^{(m+n)}(M \circ N) \simeq q^{m n+\left(\operatorname{wt}(M), n \alpha_{i}\right)} M_{0} \circ N_{0}, \\
& E_{i}^{(m+n)}(N \circ M) \simeq q^{m n+\left(\operatorname{wt}(N), m \alpha_{i}\right)} N_{0} \circ M_{0} .
\end{aligned}
$$

Using the $R$-matrix $\mathbf{r}_{M, N}$, we have

$$
M \circ N \rightarrow M \nabla N \longmapsto q^{-\Lambda(M, N)} N \circ M .
$$


Note that $E_{i}^{(m+n)}(M \nabla N) \neq 0$ since $\varepsilon_{i}(M \nabla N)=m+n$. By applying the exact functor $E_{i}^{(m+n)}$ to (3.1), we have non-zero homomorphisms

$$
q^{m n+\left(\operatorname{wt}(M), n \alpha_{i}\right)} M_{0} \circ N_{0} \rightarrow E_{i}^{(m+n)}(M \nabla N) \succ q^{-\Lambda(M, N)+m n+\left(\operatorname{wt}(N), m \alpha_{i}\right)} N_{0} \circ M_{0} .
$$

Proposition 3.5 implies that

$$
\Lambda\left(M_{0}, N_{0}\right)=\Lambda(M, N)+n\left(\alpha_{i}, \operatorname{wt}(M)\right)-m\left(\alpha_{i}, \operatorname{wt}(N)\right),
$$

which gives the assertion.

(ii) can be proved in the same manner as above.

Corollary 3.8 (cf. [7, Corollary 10.1.4]). Let $i \in I$ and $M$ a simple module. Then we have

$$
\begin{aligned}
& \Lambda(L(i), M)=\left(\alpha_{i}, \alpha_{i}\right) \varepsilon_{i}(M)+\left(\alpha_{i}, \operatorname{wt}(M)\right), \\
& \Lambda(M, L(i))=\left(\alpha_{i}, \alpha_{i}\right) \varepsilon_{i}^{*}(M)+\left(\alpha_{i}, \operatorname{wt}(M)\right) .
\end{aligned}
$$

Proof. Since

(i) $L(i)$ is real,

(ii) $\varepsilon_{i}(L(i) \nabla M)=1+\varepsilon_{i}(M)$ and $\varepsilon_{i}^{*}(M \nabla L(i))=1+\varepsilon_{i}^{*}(M)$,

we have the assertion by applying Proposition 3.7 to $L(i)$ and $M$.

\section{Determinantial modules}

From now until Lemma 4.9, we assume that $\mathrm{A}$ is arbitrary.

Let $\Lambda \in \mathrm{P}_{+}$and $\lambda=w \Lambda$ for $w \in \mathrm{W}$. Recall the cyclotomic quiver Hecke algebra $R^{\Lambda}$ in (1.1). Take a reduced expression $\underline{w}=s_{i_{1}} \cdots s_{i_{l}}$ of $w$. We define

$$
\mathrm{M}(\lambda, \Lambda):=F_{i_{1}}^{\Lambda\left(m_{1}\right)} \cdots F_{i_{l}}^{\Lambda\left(m_{l}\right)} 1,
$$

where $m_{k}=\left\langle h_{k}, s_{i_{k+1}} \cdots s_{i_{l}} \Lambda\right\rangle$ for $k=1, \ldots l$. Note that $\lambda$ is an extremal weight of $V_{q}(\Lambda)$. By Lemma $1.11, R^{\Lambda}(\lambda)$ is Morita equivalent to $\mathbf{k}$, and $\mathrm{M}(\lambda, \Lambda)$ is a simple module.

By the definition, we have

$$
[\mathrm{M}(\lambda, \Lambda)] \in V_{q}(\Lambda)_{\lambda} \subset A_{q}(\mathfrak{n})_{\lambda-\Lambda}
$$

and $[\mathrm{M}(\lambda, \Lambda)]$ is the unipotent quantum minor $D(\lambda, \Lambda)$ by this identification. Hence $\mathrm{M}(\lambda, \Lambda)$ is a self-dual simple module. Moreover the isomorphism class of $\mathrm{M}(\lambda, \Lambda)$ does not depend on the choice of reduced expression of $w$. 
Proposition 4.1. Let $\Lambda \in \mathrm{P}_{+}$. For $\lambda, \mu \in \mathrm{W} \Lambda$ with $\lambda \preceq \mu$. Then there exists a self-dual simple module $\mathrm{M}(\lambda, \mu)$ such that

$$
[\mathrm{M}(\lambda, \mu)]=D(\lambda, \mu) .
$$

Moreover, its isomorphism class is unique.

Proof. The uniqueness is obvious by Theorem 1.9. Let us write $\mu=u \Lambda$ with $u \in \mathrm{W}$. We shall argue by induction on $\ell(u)$. If $\ell(u)=0$, then it is obvious. Hence we may assume that there exists $i \in I$ such that $u^{\prime}:=s_{i} u<u$. Set $\mu^{\prime}=u^{\prime} \Lambda$. Then, by induction hypothesis, there exists a self-dual simple module $\mathrm{M}\left(\lambda, \mu^{\prime}\right)$ such that

$$
\left[\mathrm{M}\left(\lambda, \mu^{\prime}\right)\right]=D\left(\lambda, \mu^{\prime}\right) \text {. }
$$

Set $n=\left\langle h_{i}, \mu^{\prime}\right\rangle \in \mathbb{Z}_{\geq 0}$. Then we have $e_{i}^{*(n)} D\left(\lambda, \mu^{\prime}\right)=D(\lambda, \mu)$ and $e_{i}^{*(n+1)} D\left(\lambda, \mu^{\prime}\right)=0$. Hence we obtain $\varepsilon_{i}^{*}\left(\mathrm{M}\left(\lambda, \mu^{\prime}\right)\right)=n$. Moreover, $\mathrm{M}(\lambda, \mu):=E_{i}^{*(n)} \mathrm{M}\left(\lambda, \mu^{\prime}\right)$ is a simple module and it satisfies the desired condition.

One can show easily that

$$
\mathrm{W}(\mathrm{M}(\lambda, \mu)) \subset \mathrm{W}(\mathrm{M}(\lambda, \Lambda))
$$

for any $\lambda, \mu \in \mathrm{W} \Lambda$ with $\lambda \preceq \mu$.

Proposition 4.2. Let $\Lambda, \Lambda^{\prime} \in \mathrm{P}_{+}$.

(i) For $w, v \in \mathrm{W}$ with $v \leq w$, we have

$$
\mathrm{M}(w \Lambda, v \Lambda) \circ \mathrm{M}\left(w \Lambda^{\prime}, v \Lambda^{\prime}\right) \simeq q^{-\left(v \Lambda, v \Lambda^{\prime}-w \Lambda^{\prime}\right)} \mathrm{M}\left(w\left(\Lambda+\Lambda^{\prime}\right), v\left(\Lambda+\Lambda^{\prime}\right)\right) .
$$

(ii) For $\lambda, \mu \in \mathrm{W} \Lambda$ with $\lambda \preceq \mu, \mathrm{M}(\lambda, \mu)$ is real.

Proof. The assertion follows immediately from Lemma 1.6.

Lemma 4.3. Let $M$ be an $R$-module. Then we have

$$
\mathrm{W}(M)=\operatorname{wt}\left(U_{q}^{+}(\mathfrak{g})[M]\right)-\operatorname{wt}(M) .
$$

In particular, for $\Lambda \in \mathrm{P}_{+}$and $\lambda \in \mathrm{W} \Lambda$,

$$
\mathrm{W}(\mathrm{M}(\lambda, \Lambda))=\operatorname{wt}\left(U_{q}^{+}(\mathfrak{g}) u_{\lambda}\right)-\lambda
$$

where $u_{\lambda}$ is an extremal vector of weight $\lambda$ in $V_{q}(\Lambda)$.

Proof. The assertion follows from the equivalence of the following statements:

(i) $\gamma \in \mathbf{W}(M)$,

(ii) $E_{i_{n}} \cdots E_{i_{1}}[M] \neq 0$ for some $\left(i_{1}, \ldots, i_{n}\right) \in I^{\gamma}$,

(iii) $\left(U_{q}^{+}(\mathfrak{g})[M]\right)_{\mathrm{wt}(M)+\gamma} \neq 0$. 
Lemma 4.4. Let $\Lambda \in \mathrm{P}_{+}$. Let $\lambda, \mu \in \mathrm{W} \Lambda$ with $\lambda \preceq \mu$. Let $u_{\lambda} \in V(\Lambda)$ be the extremal weight vector of weight $\lambda$.

(i) If $\beta \in \mathrm{W}(\mathrm{M}(\lambda, \mu))$, then

$$
\lambda+\beta \in \operatorname{wt}\left(U_{q}^{+}(\mathfrak{g}) u_{\lambda}\right) \subset \operatorname{wt}(V(\Lambda)) .
$$

(ii) If $\gamma \in \mathrm{W}^{*}(\mathrm{M}(\lambda, \mu))$, then

$$
\mu-\gamma \in \operatorname{wt}\left(U_{q}^{+}(\mathfrak{g}) u_{\lambda}\right) \subset \operatorname{wt}(V(\Lambda)) .
$$

Proof. (i) If $\beta \in \mathrm{W}(\mathrm{M}(\lambda, \mu))$, then we have $\beta \in \mathrm{W}(M(\lambda, \Lambda))$ by (4.1). Then, Lemma 4.3 implies $\lambda+\beta \in \operatorname{wt}\left(U_{q}^{+}(\mathfrak{g}) u_{\lambda}\right)$.

(ii) If $\gamma \in \mathrm{W}^{*}(\mathrm{M}(\lambda, \mu))$, then we have $\mu-\lambda-\gamma \in \mathrm{W}(\mathrm{M}(\lambda, \mu))$. Hence (i) implies that

$$
\operatorname{wt}\left(U_{q}^{+}(\mathfrak{g}) u_{\lambda}\right) \ni \lambda+(\mu-\lambda-\gamma)=\mu-\gamma .
$$

Theorem 4.5. Let $\Lambda \in \mathrm{P}_{+}$and let $w, v \in \mathrm{W}$.

(i) Assume that $w^{\prime}, v^{\prime} \in \mathrm{W}$ satisfy

$$
w \geq w^{\prime} \geq v^{\prime} \geq v, \quad \ell(w)=\ell\left(w^{\prime}\right)+\ell\left(w^{\prime-1} w\right), \quad \ell\left(v^{\prime}\right)=\ell(v)+\ell\left(v^{-1} v^{\prime}\right) .
$$

Then we have $\mathscr{C}_{w^{\prime}, v^{\prime}} \subset \mathscr{C}_{w, v}$.

(ii) We have

$$
\mathrm{M}(w \Lambda, v \Lambda) \in \mathscr{C}_{w, v}
$$

Proof. (i) follows from $\Delta_{+} \cap w^{\prime} \Delta_{-} \subset \Delta_{+} \cap w \Delta_{-}$and $\Delta_{+} \cap v^{\prime} \Delta_{+} \subset \Delta_{+} \cap v \Delta_{+}$.

(ii) Let $M=\mathrm{M}(w \Lambda, v \Lambda)$. We take $\beta \in \mathrm{W}(M)$. Then, Lemma 4.4 (i) tells that

$$
w \Lambda+\beta \in \operatorname{wt}(V(\Lambda)) .
$$

Since $\Lambda+w^{-1} \beta \in \operatorname{wt}(V(\Lambda))$ and $\Lambda$ is the highest weight of $V(\Lambda)$, we have $w^{-1} \beta \in Q_{-}$, which implies that $\beta \in w Q_{-}$. Thus we have $M \in \mathscr{C}_{w}$.

We now take $\gamma \in \mathbf{W}^{*}(M)$. It follows from Lemma 4.4 (ii) that

$$
v \Lambda-\gamma \in \operatorname{wt}(V(\Lambda)) .
$$

As $\Lambda-v^{-1} \gamma \in \operatorname{wt}(V(\Lambda))$, we have $v^{-1} \gamma \in \mathrm{Q}_{+}$. Thus we have $\gamma \in v \mathrm{Q}_{+}$, which implies $M \in \mathscr{C}_{*, v}$.

The following proposition is proved in [7, Theorem 10.3.1] when the quiver Hecke algebra is symmetric. 
Proposition 4.6. Let $\Lambda \in \mathrm{P}_{+}$and assume that $\lambda, \lambda^{\prime}, \lambda^{\prime \prime} \in \mathrm{W} \Lambda$ satisfy $\lambda \preceq \lambda^{\prime} \preceq \lambda^{\prime \prime}$. Then there is an epimorphism

$$
\mathrm{M}\left(\lambda, \lambda^{\prime}\right) \circ \mathrm{M}\left(\lambda^{\prime}, \lambda^{\prime \prime}\right) \rightarrow \mathrm{M}\left(\lambda, \lambda^{\prime \prime}\right) .
$$

Proof. Assume that $w, x, y, z \in \mathrm{W}$ such that $z \leq y \leq x \leq w$. Then Theorem 4.5 implies that

$$
\begin{aligned}
& \mathrm{W}^{*}(\mathrm{M}(x \Lambda, y \Lambda)) \subset \mathrm{Q}_{+} \cap y \mathrm{Q}_{+}, \mathrm{W}(\mathrm{M}(y \Lambda, z \Lambda)) \subset \mathrm{Q}_{+} \cap y \mathrm{Q}_{-}, \\
& \mathrm{W}^{*}(\mathrm{M}(y \Lambda, z \Lambda)) \subset \mathrm{Q}_{+} \cap z \mathrm{Q}_{+}, \mathrm{W}(\mathrm{M}(z \Lambda, w \Lambda)) \subset \mathrm{Q}_{+} \cap z \mathrm{Q}_{-} .
\end{aligned}
$$

It implies that $(\mathrm{M}(x \Lambda, y \Lambda), \mathrm{M}(y \Lambda, z \Lambda))$ and $(\mathrm{M}(x \Lambda, y \Lambda), \mathrm{M}(y \Lambda, z \Lambda), \mathrm{M}(z \Lambda, w \Lambda))$ are unmixed. Thus we conclude that

$$
\mathrm{M}(x \Lambda, y \Lambda) \circ \mathrm{M}(y \Lambda, z \Lambda) \text { and } \mathrm{M}(x \Lambda, y \Lambda) \circ \mathrm{M}(y \Lambda, z \Lambda) \circ \mathrm{M}(z \Lambda, w \Lambda) \text { have simple heads. }
$$

(i) Case $\lambda^{\prime \prime}=\Lambda$. Set $\lambda^{\prime}=y \Lambda$. We shall prove the assertion by induction on $\ell(y)$. When $y=1$ it is obvious. Otherwise take $i \in I$ such that $y^{\prime}:=s_{i} y<y$. Then

$$
\mathrm{M}(\lambda, y \Lambda) \circ \mathrm{M}\left(y \Lambda, y^{\prime} \Lambda\right) \circ \mathrm{M}\left(y^{\prime} \Lambda, \Lambda\right)
$$

has a simple head. By the definition we have an epimorphism $\mathrm{M}(\lambda, y \Lambda) \circ \mathrm{M}\left(y \Lambda, y^{\prime} \Lambda\right) \rightarrow$ $\mathrm{M}\left(\lambda, y^{\prime} \Lambda\right)$ since $\mathrm{M}\left(y \Lambda, y^{\prime} \Lambda\right) \simeq L\left(i^{n}\right)$ with $n=\left\langle h_{i}, y^{\prime} \Lambda\right\rangle$ and $\mathrm{M}(\lambda, y \Lambda) \simeq E_{i}^{*(n)} \mathrm{M}\left(\lambda, y^{\prime} \Lambda\right)$. By induction hypothesis there exists an epimorphism $\mathrm{M}\left(\lambda, y^{\prime} \Lambda\right) \circ \mathrm{M}\left(y^{\prime} \Lambda, \Lambda\right) \rightarrow \mathrm{M}(\lambda, \Lambda)$. Thus we have an epimorphism $\mathrm{M}(\lambda, y \Lambda) \circ \mathrm{M}\left(y \Lambda, y^{\prime} \Lambda\right) \circ \mathrm{M}\left(y^{\prime} \Lambda, \Lambda\right) \rightarrow \mathrm{M}(\lambda, \Lambda)$. Hence $\mathrm{M}(\lambda, \Lambda)$ is the simple head of $\mathrm{M}(\lambda, y \Lambda) \circ \mathrm{M}\left(y \Lambda, y^{\prime} \Lambda\right) \circ \mathrm{M}\left(y^{\prime} \Lambda, \Lambda\right)$. On the other hand we have an epimorphism

$$
\mathrm{M}(\lambda, y \Lambda) \circ \mathrm{M}\left(y \Lambda, y^{\prime} \Lambda\right) \circ \mathrm{M}\left(y^{\prime} \Lambda, \Lambda\right) \rightarrow \mathrm{M}(\lambda, y \Lambda) \circ \mathrm{M}(y \Lambda, \Lambda) .
$$

Hence $\mathrm{M}(\lambda, \Lambda)$ is equal to the simple head of $\mathrm{M}(\lambda, y \Lambda) \circ \mathrm{M}(y \Lambda, \Lambda)$.

(ii) Reduction to the case $\lambda^{\prime \prime}=\Lambda$. Assume that $\lambda^{\prime \prime} \neq \Lambda$. Then there is $i \in I$ such that $n:=-\left\langle h_{i}, \lambda^{\prime \prime}\right\rangle>0$. By induction on $h t\left(\Lambda-\lambda^{\prime \prime}\right)$, we may assume that the assertion holds for $s_{i} \lambda^{\prime \prime}$. Therefore, we have a homomorphism

$$
\begin{aligned}
\mathrm{M}\left(\lambda, \lambda^{\prime}\right) \circ \mathrm{M}\left(\lambda^{\prime}, \lambda^{\prime \prime}\right) & \simeq \mathrm{M}\left(\lambda, \lambda^{\prime}\right) \circ\left(E_{i}^{*(n)} \mathrm{M}\left(\lambda^{\prime}, s_{i} \lambda^{\prime \prime}\right)\right) \\
& \rightarrow E_{i}^{*(n)}\left(\mathrm{M}\left(\lambda, \lambda^{\prime}\right) \circ \mathrm{M}\left(\lambda^{\prime} s_{i} \lambda^{\prime \prime}\right)\right) \\
& \rightarrow E_{i}^{*(n)} \mathrm{M}\left(\lambda, s_{i} \lambda^{\prime \prime}\right) \\
& \simeq \mathrm{M}\left(\lambda, \lambda^{\prime \prime}\right) .
\end{aligned}
$$

It is obvious that the composition does not vanish, and hence it is an epimorphism.

Corollary 4.7. Let $\Lambda \in \mathrm{P}_{+}$. 
(i) Let $i \in I$ and $v \in \mathrm{W}$ such that $v s_{i}\left\langle v\right.$. If $n:=\left\langle h_{i}, \Lambda\right\rangle>0$, then $\mathrm{M}\left(v \Lambda, v s_{i} \Lambda\right)$ is $a \preceq \underline{v}$-cuspidal $R\left(-n v \alpha_{i}\right)$-module, where $\preceq \underline{v}$ is a convex order corresponding to a reduced expression $\underline{v}$ given in Proposition 1.24.

(ii) We take a reduced expression $\underline{w}=s_{i_{1}} \cdots s_{i_{\ell}}$ of $w \in \mathrm{W}$. Then the sequence obtained from $\left(\mathrm{M}\left(w_{\ell} \Lambda, w_{\ell-1} \Lambda\right), \ldots, \mathrm{M}\left(w_{1} \Lambda, w_{0} \Lambda\right)\right)$ by removing $\mathrm{M}\left(w_{k} \Lambda, w_{k-1} \Lambda\right)$ such that $w_{k} \Lambda=w_{k-1} \Lambda$, is a $\preceq \underline{w}$-cuspidal decomposition of $\mathrm{M}(w \Lambda, \Lambda)$, where $w_{k}=s_{i_{1}} \cdots s_{i_{k}}$ for $k=1, \ldots, \ell$.

Proof. (i) Set $v^{\prime}=v s_{i}$. Then $-\operatorname{wt}\left(\mathrm{M}\left(v \Lambda, v s_{i} \Lambda\right)\right)=n v^{\prime} \alpha_{i}$ and Theorem 4.5 implies that $\mathrm{W}\left(\mathrm{M}\left(v \Lambda, v s_{i} \Lambda\right)\right) \subset \operatorname{span}_{\mathbb{R}_{\geq 0}}\left(\Delta_{+} \cap v \Delta_{-}\right)=\operatorname{span}_{\mathbb{R}_{\geq 0}}\left(\left\{\beta \in \Delta_{+} \mid \beta \preceq \underline{v} v^{\prime} \alpha_{i}\right\}\right)$. Hence $\mathrm{M}\left(v \Lambda, v s_{i} \Lambda\right)$ is a $\preceq \underline{v}$-cuspidal $\bar{R}\left(n v^{\prime} \alpha_{i}\right)$-module.

(ii) By Proposition 4.6, there exists an epimorphism

$$
\mathrm{M}\left(w_{\ell} \Lambda, w_{\ell-1} \Lambda\right) \circ \cdots \circ \mathrm{M}\left(w_{1} \Lambda, w_{0} \Lambda\right) \rightarrow \mathrm{M}(w \Lambda, \Lambda) .
$$

Hence (ii) follows from (i).

Let $w, v \in \mathrm{W}$ with $v \leq w$ and fix a reduced expression $\underline{w}=s_{i_{1}} s_{i_{2}} \cdots s_{i_{l}}$ of $w$. For $k=1, \ldots, l$, we set

$$
w_{\leq k}:=s_{i_{1}} \cdots s_{i_{k}}, \quad w_{\geq k+1}:=w_{\leq k}^{-1} w .
$$

We shall define $v_{\leq k}$ and $v_{\geq k}$ for $k=1, \ldots, l$ by

$$
\begin{aligned}
& \text { (i) } v_{\geq k}=\left(v_{\leq k-1}\right)^{-1} v, \\
& \text { (ii) } v_{\leq k}= \begin{cases}v_{\leq k-1} s_{i_{k}} & \text { if } s_{i_{k}} v_{\geq k}<v_{\geq k}, \\
v_{\leq k-1} & \text { if } s_{i_{k}} v_{\geq k}>v_{\geq k} .\end{cases}
\end{aligned}
$$

Here we set $w_{\leq 0}=v_{\leq 0}=\mathrm{id} \in \mathrm{W}$. Note that $w_{\leq l}=w, v_{\leq l}=v, v_{\leq k} \leq w_{\leq k}, v_{\geq k} \leq w_{\geq k}$ and $\ell(v)=\ell\left(v_{\leq k-1}\right)+\ell\left(v_{\geq k}\right)$ for $k=1, \ldots, l$.

Proposition 4.8. Let $\Lambda \in \mathrm{P}_{+}$and $w, v \in \mathrm{W}$ with $v \leq w$. We fix a reduced expression $\underline{w}=s_{i_{1}} \cdots s_{i_{l}}$. For $k=0,1, \ldots, l$,

$$
\mathrm{M}\left(w_{\leq k} \Lambda, v_{\leq k} \Lambda\right) \in \mathscr{C}_{w, v}
$$

where $w_{\leq k}$ and $v_{\leq k}$ are defined in (4.2) and (4.3).

Proof. Note that $\mathrm{M}\left(w_{\leq k} \Lambda, v_{\leq k} \Lambda\right) \in \mathscr{C}_{w_{\leq k}} \subset \mathscr{C}_{w}$ follows from Theorem 4.5. Hence it remains to prove $\mathrm{W}^{*}\left(\mathrm{M}\left(w_{\leq k} \Lambda, v_{\leq k} \Lambda\right)\right) \in v \mathrm{Q}_{+}$.

Assume that $\beta \in \mathrm{W}^{*}\left(\mathrm{M}\left(w_{\leq k} \Lambda, v_{\leq k} \Lambda\right)\right)$. Then Lemma 4.4 (ii) implies that

$$
v_{\leq k} \Lambda-\beta \in \operatorname{wt}\left(U_{q}^{+}(\mathfrak{g}) u_{w_{\leq k} \Lambda}\right) .
$$


Hence it is enough to show that

if $\beta \in \mathrm{Q}$ satisfies condition (4.4), then we have $\beta \in v \mathrm{Q}_{+}$.

We shall prove it by induction on $\ell(w)+\ell(v)$.

Since it is obvious when $k=0$, let us assume that $k>0$. Set $i=i_{1}$.

(1) Case $s_{i} v>v$. We have $\alpha_{i} \in v \Delta_{+}$. Set $w^{\prime}:=s_{i} w<w$ and $v^{\prime}:=v$. We define $w_{\leq k}^{\prime}$ and $v_{\leq k}^{\prime}$ with respect to the reduced expression $w^{\prime}=s_{i_{2}} \cdots s_{i_{l}}$ and $v^{\prime}$. Then we have $w_{\leq k}=s_{i} w_{\leq k-1}^{\prime}$ and $v_{\leq k}=v_{\leq k-1}^{\prime}$. Hence $v_{\leq k-1}^{\prime} \Lambda-\beta \in \operatorname{wt}\left(U_{q}^{+}(\mathfrak{g}) u_{s_{i} w_{\leq k-1}^{\prime} \Lambda}\right) \subset$ $\operatorname{wt}\left(U_{q}^{+}(\mathfrak{g}) u_{w_{\leq k-1}^{\prime} \Lambda}\right)-\mathbb{Z}_{\geq 0} \alpha_{i}$. Hence for some $m \in \mathbb{Z}_{\geq 0}$ we have $v_{\leq k-1}^{\prime} \Lambda-\left(\beta-m \alpha_{i}\right) \in$ $\operatorname{wt}\left(U_{q}^{+}(\mathfrak{g}) u_{w_{\leq k-1}^{\prime} \Lambda}\right)$. Then, induction hypothesis implies that $\beta-m \alpha_{i} \in v^{\prime} \mathrm{Q}_{+}=v \mathrm{Q}_{+}$. Hence we obtain $\beta \in v \mathrm{Q}_{+}+m \alpha_{i} \subset v \mathrm{Q}_{+}$.

(2) Case $s_{i} v<v$. Set $w^{\prime}:=w$ and $v^{\prime}:=s_{i} v<v$. We define $w_{\leq k}^{\prime}$ and $v_{\leq k}^{\prime}$ with respect to the reduced expression $w^{\prime}=s_{i_{1}} \cdots s_{i_{l}}$ and $v^{\prime}$. Then we have $w_{\leq k}=w_{\leq k}^{\prime}$ and $v_{\leq k}=s_{i} v_{\leq k}^{\prime}$. Hence $s_{i} v_{\leq k}^{\prime} \Lambda-\beta \in \operatorname{wt}\left(U_{q}^{+}(\mathfrak{g}) u_{w_{\leq k}^{\prime} \Lambda}\right)$.

Since $s_{i} w_{\leq k}^{\prime}<w_{\leq k}^{\prime}$, the set $\operatorname{wt}\left(U_{q}^{+}(\mathfrak{g}) u_{w_{\leq k}^{\prime} \Lambda}\right)$ is invariant by the action of $s_{i} \in \mathrm{W}$. Hence we have

$$
v_{\leq k}^{\prime} \Lambda-s_{i} \beta \in \operatorname{wt}\left(U_{q}^{+}(\mathfrak{g}) u_{w_{\leq k}^{\prime} \Lambda}\right) .
$$

Then induction hypothesis implies that $s_{i} \beta \in v^{\prime} \mathbf{Q}_{+}$. Hence we obtain $\beta \in s_{i} v^{\prime} \mathbf{Q}_{+}=$ $v \mathrm{Q}_{+}$.

We assume that $R(\beta)$ is symmetric in the rest of this section.

Lemma 4.9. Let $i \in I$ and let $\lambda, \mu \in \mathrm{W} \Lambda$ with $\lambda \preceq \mu$ for $\Lambda \in \mathrm{P}_{+}$. If $\left\langle h_{i}, \lambda\right\rangle \leq 0$ and $\left\langle h_{i}, \mu\right\rangle \geq 0$, then $\mathrm{M}(\lambda, \mu)$ strongly commutes with $L(i)$.

Proof. By the construction, we have

$$
\varepsilon_{i}(\mathrm{M}(\lambda, \mu))=-\left\langle h_{i}, \lambda\right\rangle, \quad \varepsilon_{i}^{*}(\mathrm{M}(\lambda, \mu))=\left\langle h_{i}, \mu\right\rangle, \quad \operatorname{wt}(\mathrm{M}(\lambda, \mu))=\lambda-\mu .
$$

By Corollary 3.8, we have

$$
\begin{aligned}
& \Lambda(L(i), \mathrm{M}(\lambda, \mu))+\Lambda(\mathrm{M}(\lambda, \mu), L(i)) \\
& \quad=\left(\alpha_{i}, \alpha_{i}\right) \varepsilon_{i}(\mathrm{M}(\lambda, \mu))+\left(\alpha_{i}, \operatorname{wt}(\mathrm{M}(\lambda, \mu))\right)+\left(\alpha_{i}, \alpha_{i}\right) \varepsilon_{i}^{*}(\mathrm{M}(\lambda, \mu))+\left(\alpha_{i}, \operatorname{wt}(\mathrm{M}(\lambda, \mu))\right) \\
& \quad=0,
\end{aligned}
$$

which yields the assertion by Proposition 3.5.

Theorem 4.10. Let $\Lambda, \Lambda^{\prime} \in \mathrm{P}_{+}$and $w, v \in \mathrm{W}$ with $v \leq w$. We fix a reduced expression $\underline{w}=s_{i_{1}} \cdots s_{i_{l}}$. For $1 \leq k \leq j \leq l$,

$$
\mathrm{M}\left(w_{\leq j} \Lambda, v_{\leq j} \Lambda\right) \text { and } \mathrm{M}\left(w_{\leq k} \Lambda^{\prime}, v_{\leq k} \Lambda^{\prime}\right) \text { strongly commute, }
$$


where $w_{\leq k}$ and $v_{\leq k}$ are defined in (4.2) and (4.3).

Proof. By replacing $w$ and $v$ with $w_{\leq j}$ and $v_{\leq j}$, it is enough to show that $\mathrm{M}(w \Lambda, v \Lambda)$ and $\mathrm{M}\left(w_{\leq k} \Lambda^{\prime}, v_{\leq k} \Lambda^{\prime}\right)$ strongly commute. We shall show it by induction on $l$.

Let $i=i_{1}$. We assume first that $v_{\leq 1}=\mathrm{id}$. Then $s_{i} v>v$ and $s_{i} v_{\leq k}>v_{\leq k}$ for any $k$. As $\left\langle h_{i}, w \Lambda\right\rangle \leq 0$ and $\left\langle h_{i}, v \Lambda\right\rangle \geq 0, \mathrm{M}(w \Lambda, v \Lambda)$ and $\mathrm{M}\left(w_{\leq k} \Lambda^{\prime}, v_{\leq k} \Lambda^{\prime}\right)$ strongly commute with $L(i)$ by Lemma 4.9. As

$$
\begin{aligned}
\widetilde{E}_{i}^{\max } \mathrm{M}(w \Lambda, v \Lambda) & \simeq \mathrm{M}\left(s_{i} w \Lambda, v \Lambda\right), \\
\widetilde{E}_{i}^{\max } \mathrm{M}\left(w_{\leq k} \Lambda^{\prime}, v_{\leq k} \Lambda^{\prime}\right) & \simeq \mathrm{M}\left(s_{i} w_{\leq k} \Lambda^{\prime}, v_{\leq k} \Lambda^{\prime}\right),
\end{aligned}
$$

by the induction hypothesis on $l, \widetilde{E}_{i}^{\max } \mathrm{M}(w \Lambda, v \Lambda)$ and $\widetilde{E}_{i}^{\max } \mathrm{M}\left(w_{\leq k} \Lambda^{\prime}, v_{\leq k} \Lambda^{\prime}\right)$ strongly commute. Hence $\mathrm{M}(w \Lambda, v \Lambda)$ and $\mathrm{M}\left(w_{\leq k} \Lambda^{\prime}, v_{\leq k} \Lambda^{\prime}\right)$ strongly commute by Lemma 3.6.

Suppose $v_{\leq 1}=s_{i}$. Letting $v^{\prime}=s_{i} v$, we have $v_{\leq k}^{\prime}=s_{i} v_{\leq k}$ for $k=1, \ldots, l$. Since $s_{i} v^{\prime}>v^{\prime}$, it follows from the above that $\mathrm{M}\left(w \Lambda, v^{\prime} \Lambda\right)$ and $\mathrm{M}\left(w_{\leq k} \Lambda^{\prime}, v_{\leq k}^{\prime} \Lambda^{\prime}\right)$ strongly commute. Therefore, Lemma 3.1 implies that $\mathrm{M}(w \Lambda, v \Lambda)$ and $\mathrm{M}\left(w_{\leq k} \Lambda^{\prime}, v_{\leq k} \Lambda^{\prime}\right)$ strongly commute.

Lemma 4.11. Let $i \in I, \lambda, \mu \in \mathrm{W} \Lambda$ and $\lambda^{\prime}, \mu^{\prime} \in \mathrm{W} \Lambda^{\prime}$ such that

$$
\left\langle h_{i}, \lambda^{\prime}\right\rangle \leq 0, \quad\left\langle h_{i}, \mu\right\rangle \geq 0, \quad s_{i} \lambda \preceq \mu, \quad s_{i} \lambda^{\prime} \preceq \mu^{\prime} .
$$

(i) If $\left\langle h_{i}, \lambda\right\rangle \leq 0$, then we have

$\Lambda\left(\mathrm{M}(\lambda, \mu), \mathrm{M}\left(\lambda^{\prime}, \mu^{\prime}\right)\right)-\Lambda\left(\mathrm{M}\left(s_{i} \lambda, \mu\right), \mathrm{M}\left(s_{i} \lambda^{\prime}, \mu^{\prime}\right)\right)=\left(\lambda+\mu, \mu^{\prime}-\lambda^{\prime}\right)-\left(s_{i} \lambda+\mu, \mu^{\prime}-s_{i} \lambda^{\prime}\right)$.

(ii) If $\left\langle h_{i}, \mu^{\prime}\right\rangle \geq 0$, then we have

$\Lambda\left(\mathrm{M}(\lambda, \mu), \mathrm{M}\left(\lambda^{\prime}, \mu^{\prime}\right)\right)-\Lambda\left(\mathrm{M}\left(\lambda, s_{i} \mu\right), \mathrm{M}\left(\lambda^{\prime}, s_{i} \mu^{\prime}\right)\right)=\left(\lambda+\mu, \mu^{\prime}-\lambda^{\prime}\right)-\left(\lambda+s_{i} \mu, s_{i} \mu^{\prime}-\lambda^{\prime}\right)$.

Proof. We set

$$
\Lambda_{\lambda, \mu, \lambda^{\prime}, \mu^{\prime}}:=\Lambda\left(\mathrm{M}(\lambda, \mu), \mathrm{M}\left(\lambda^{\prime}, \mu^{\prime}\right)\right) .
$$

(i) By Lemma 4.9 and Proposition 4.2, $\mathrm{M}(\lambda, \mu)$ is real and strongly commutes with $L(i)$. Let $m=-\left\langle h_{i}, \lambda\right\rangle$ and $n=-\left\langle h_{i}, \lambda^{\prime}\right\rangle$. It follows from Lemma 3.2 and Proposition 3.7 that

$$
\begin{aligned}
\Lambda_{\lambda, \mu, \lambda^{\prime}, \mu^{\prime}}-\Lambda_{s_{i} \lambda, \mu, s_{i} \lambda^{\prime}, \mu^{\prime}} & =-n\left(\alpha_{i}, \operatorname{wt}(\mathrm{M}(\lambda, \mu))\right)+m\left(\alpha_{i}, \operatorname{wt}\left(\mathrm{M}\left(\lambda^{\prime}, \mu^{\prime}\right)\right)\right) \\
& =-n\left(\alpha_{i}, \lambda-\mu\right)+m\left(\alpha_{i}, \lambda^{\prime}-\mu^{\prime}\right)
\end{aligned}
$$


and

$$
\begin{aligned}
\left(\lambda+\mu, \mu^{\prime}\right. & \left.-\lambda^{\prime}\right)-\left(s_{i} \lambda+\mu, \mu^{\prime}-s_{i} \lambda^{\prime}\right) \\
& =\left(\lambda+\mu, \mu^{\prime}-\lambda^{\prime}\right)-\left(\lambda+\mu+m \alpha_{i}, \mu^{\prime}-\lambda^{\prime}-n \alpha_{i}\right) \\
& =-m\left(\alpha_{i}, \mu^{\prime}-\lambda^{\prime}\right)+n\left(\alpha_{i}, \lambda+\mu\right)+m n\left(\alpha_{i}, \alpha_{i}\right) \\
& =m\left(\alpha_{i}, \lambda^{\prime}-\mu^{\prime}\right)-n\left(\alpha_{i}, \lambda-\mu\right)+2 n\left(\alpha_{i}, \lambda\right)+m n\left(\alpha_{i}, \alpha_{i}\right) \\
& =m\left(\alpha_{i}, \lambda^{\prime}-\mu^{\prime}\right)-n\left(\alpha_{i}, \lambda-\mu\right) .
\end{aligned}
$$

(ii) Note that $\mathrm{M}\left(\lambda^{\prime}, \mu^{\prime}\right)$ is real and strongly commutes with $L(i)$ by Lemma 4.9 and Proposition 4.2. Let $p=\left\langle h_{i}, \mu\right\rangle$ and $q=\left\langle h_{i}, \mu^{\prime}\right\rangle$. By Lemma 3.2 and Proposition 3.7, we obtain

$$
\begin{aligned}
\Lambda_{\lambda, \mu, \lambda^{\prime}, \mu^{\prime}}-\Lambda_{\lambda, s_{i} \mu, \lambda^{\prime}, s_{i} \mu^{\prime}} & =q\left(\alpha_{i}, \operatorname{wt}(\mathrm{M}(\lambda, \mu))\right)-p\left(\alpha_{i}, \operatorname{wt}\left(\mathrm{M}\left(\lambda^{\prime}, \mu^{\prime}\right)\right)\right) \\
& =q\left(\alpha_{i}, \lambda-\mu\right)-p\left(\alpha_{i}, \lambda^{\prime}-\mu^{\prime}\right) .
\end{aligned}
$$

and

$$
\begin{aligned}
\left(\lambda+\mu, \mu^{\prime}\right. & \left.-\lambda^{\prime}\right)-\left(\lambda+s_{i} \mu, s_{i} \mu^{\prime}-\lambda^{\prime}\right) \\
& =\left(\lambda+\mu, \mu^{\prime}-\lambda^{\prime}\right)-\left(\lambda+\mu-p \alpha_{i}, \mu^{\prime}-\lambda^{\prime}-q \alpha_{i}\right) \\
& =q\left(\lambda+\mu, \alpha_{i}\right)+p\left(\alpha_{i}, \mu^{\prime}-\lambda^{\prime}\right)-p q\left(\alpha_{i}, \alpha_{i}\right) \\
& =q\left(\lambda-\mu, \alpha_{i}\right)-p\left(\alpha_{i}, \lambda^{\prime}-\mu^{\prime}\right)+2 q\left(\alpha_{i}, \mu\right)-p q\left(\alpha_{i}, \alpha_{i}\right) \\
& =q\left(\lambda-\mu, \alpha_{i}\right)-p\left(\alpha_{i}, \lambda^{\prime}-\mu^{\prime}\right) .
\end{aligned}
$$

Theorem 4.12. Let $\Lambda, \Lambda^{\prime} \in \mathrm{P}_{+}$and $w, v \in \mathrm{W}$ with $v \leq w$. We fix a reduced expression $\underline{w}=s_{i_{1}} \cdots s_{i_{l}}$. For $k=0,1, \ldots, l$, we have

$$
\Lambda\left(\mathrm{M}(w \Lambda, v \Lambda), \mathrm{M}\left(w_{\leq k} \Lambda^{\prime}, v_{\leq k} \Lambda^{\prime}\right)\right)=\left(w \Lambda+v \Lambda, v_{\leq k} \Lambda^{\prime}-w_{\leq k} \Lambda^{\prime}\right) .
$$

Proof. We use induction on $l:=\ell(w)+\ell(v)$. As it is obvious that the assertion holds if either $l=0$ or $k=0$, we assume that $l>0$ and $k>0$. Let $i=i_{1}$. Then we have

$$
\left\langle h_{i}, w \Lambda\right\rangle \leq 0, \quad\left\langle h_{i}, w_{\leq k} \Lambda\right\rangle \leq 0 .
$$

Suppose that $s_{i} v>v$. Note that $\left\langle h_{i}, v \Lambda^{\prime}\right\rangle \geq 0$. Let $\tilde{w}=s_{i} w$ and $\tilde{v}=v$. Since $\tilde{w}_{\leq k-1}=s_{i} w_{\leq k}$ and $\tilde{v}_{\leq k-1}=v_{\leq k}$, the induction hypothesis implies

$$
\begin{aligned}
\Lambda\left(\mathrm{M}\left(s_{i} w \Lambda, v \Lambda\right), \mathrm{M}\left(s_{i} w_{\leq k} \Lambda^{\prime}, v_{\leq k} \Lambda^{\prime}\right)\right) & =\Lambda\left(\mathrm{M}(\tilde{w} \Lambda, \tilde{v} \Lambda), \mathrm{M}\left(\tilde{w}_{\leq k-1} \Lambda^{\prime}, \tilde{v}_{\leq k-1} \Lambda^{\prime}\right)\right) \\
& =\left(s_{i} w \Lambda+v \Lambda, v_{\leq k} \Lambda^{\prime}-s_{i} w_{\leq k} \Lambda^{\prime}\right) .
\end{aligned}
$$


By Lemma 4.11, we have

$$
\Lambda\left(\mathrm{M}(w \Lambda, v \Lambda), \mathbf{M}\left(w_{\leq k} \Lambda^{\prime}, v_{\leq k} \Lambda^{\prime}\right)\right)=\left(w \Lambda+v \Lambda, v_{\leq k} \Lambda^{\prime}-w_{\leq k} \Lambda^{\prime}\right) .
$$

We now suppose that $s_{i} v<v$. Note that $\left\langle h_{i}, s_{i} v \Lambda^{\prime}\right\rangle \geq 0$. Let $\hat{w}=w$ and $\hat{v}=s_{i} v$. Then $\hat{w}_{\leq k}=w_{\leq k}$ and $\hat{v}_{\leq k}=s_{i} v_{\leq k}$, so we have

$$
\begin{aligned}
\Lambda\left(\mathrm{M}\left(w \Lambda, s_{i} v \Lambda\right), \mathrm{M}\left(w_{\leq k} \Lambda^{\prime}, s_{i} v_{\leq k} \Lambda^{\prime}\right)\right) & =\Lambda\left(\mathrm{M}(\hat{w} \Lambda, \hat{v} \Lambda), \mathrm{M}\left(\hat{w}_{\leq k} \Lambda^{\prime}, \hat{v}_{\leq k} \Lambda^{\prime}\right)\right) \\
& =\left(w \Lambda+s_{i} v \Lambda, s_{i} v_{\leq k} \Lambda^{\prime}-w_{\leq k} \Lambda^{\prime}\right)
\end{aligned}
$$

by the induction hypothesis. Therefore, Lemma 4.11 implies that

$$
\Lambda\left(\mathrm{M}(w \Lambda, v \Lambda), \mathbf{M}\left(w_{\leq k} \Lambda^{\prime}, v_{\leq k} \Lambda^{\prime}\right)\right)=\left(w \Lambda+v \Lambda, v_{\leq k} \Lambda^{\prime}-w_{\leq k} \Lambda^{\prime}\right) .
$$

\section{Finite ADE TYPES}

In this section, we assume that $\mathrm{A}$ is of finite $A D E$ type. Then any corresponding quiver Hecke algebra $R$ is isomorphic to a symmetric quiver Hecke algebra. We assume further that the base field $\mathbf{k}$ is of characteristic 0 . Under this assumption, the set of the isomorphism classes of simple $R$-modules corresponds to the upper global basis of $A_{q}(\mathfrak{n})([22,25])$.

For $i \in I$, we define ${ }_{i} R$-mod (resp. $R_{i}$-mod) to be the full subcategory of $R$-mod whose objects $M$ satisfy $\varepsilon_{i}(M)=0$ (resp. $\left.\varepsilon_{i}^{*}(M)=0\right)$. Note that the categories ${ }_{i} R$-mod and $R_{i}$-mod are stable under taking subquotients, extensions, convolution products and grading shifts. It was proved in [11, Proposition 3.5] that there exist reflection functors

$$
\begin{aligned}
\mathcal{T}_{i}: R_{i}(\beta)-\bmod \stackrel{\sim}{\longrightarrow}{ }_{i} R\left(s_{i} \beta\right)-\bmod , \\
\mathcal{T}_{i}{ }^{*}:{ }_{i} R(\beta)-\bmod \stackrel{\sim}{\longrightarrow} R_{i}\left(s_{i} \beta\right)-\bmod ,
\end{aligned}
$$

which are equivalences of categories commuting with the grading shift functor. Note that, by [11, Proposition 3.5]

$\mathcal{T}_{i}$ and $\mathcal{T}_{i}^{*}$ are quasi-inverse.

The functors $\mathcal{T}_{i}$ and $\mathcal{T}_{i}{ }^{*}$ are counterparts of the Saito crystal reflections on the crystals [23], which are defined by

$$
\begin{aligned}
T_{i}: B_{i}(\infty) \longrightarrow{ }_{i} B(\infty), & b & \mapsto \tilde{f}_{i}^{*} \varphi_{i}(b) \widetilde{e}_{i}^{\varepsilon_{i}(b)} b, \\
T_{i}^{*}:{ }_{i} B(\infty) \longrightarrow B_{i}(\infty), & b & \mapsto \tilde{f}_{i}^{\varphi_{i}^{*}(b)} \widetilde{e}_{i}^{* \varepsilon_{i}^{*}(b)} b,
\end{aligned}
$$


where ${ }_{i} B(\infty)=\left\{b \in B(\infty) \mid \varepsilon_{i}(b)=0\right\}$ and $B_{i}(\infty)=\left\{b \in B(\infty) \mid \varepsilon_{i}^{*}(b)=0\right\}$. Letting $L(b)$ be the self-dual simple $R$-module corresponding to $b \in B(\infty)$, it was shown in [11, Theorem 3.6] that

$$
\mathcal{T}_{i}(L(b)) \simeq L\left(T_{i} b\right) \quad \text { and } \quad \mathcal{T}_{i}^{*}\left(L\left(b^{\prime}\right)\right) \simeq L\left(T_{i}^{*} b^{\prime}\right)
$$

for $b \in B_{i}(\infty)$ and $b^{\prime} \in{ }_{i} B(\infty)$.

For a reduced expression $\underline{w}$ of $w \in W$, let us recall that $\preceq \underline{w}$ denotes a convex order corresponding to $\underline{w}$ defined in Proposition 1.24. We consider a reduced expression $\underline{w_{0}}=s_{i_{1}} \cdots s_{i_{\ell}}$ of the longest element $w_{0} \in \mathrm{W}$. If we set $L_{k}$ to be the $\preceq \underline{w_{0}}$-cuspidal module corresponding to the positive root $\beta_{k}=s_{i_{1}} \cdots s_{i_{k-1}}\left(\alpha_{i_{k}}\right)$, we have

$$
L_{k} \simeq L\left(T_{i_{1}}^{*} T_{i_{2}}^{*} \cdots T_{i_{k-1}}^{*}\left(f_{i_{k}}\right)\right) \simeq \mathcal{T}_{i_{1}}^{*} \mathcal{T}_{i_{2}}^{*} \cdots \mathcal{T}_{i_{k-1}}^{*} L\left(i_{k}\right)
$$

Further, it was shown in [11, Lemma 4.2] that, for $1 \leq p<q \leq \ell$ and $\left(t_{p}, \ldots, t_{q}\right) \in$ $\mathbb{Z}_{\geq 0}^{q-p+1}$

$$
\begin{aligned}
& \mathcal{T}_{p, q-1}^{*}\left(L\left(i_{q}^{t_{q}}\right)\right) \circ \mathcal{T}_{p, q-2}^{*}\left(L\left(i_{q-1}^{t_{q-1}}\right)\right) \circ \cdots \circ \mathcal{T}_{p, p}^{*}\left(L\left(i_{p+1}^{t_{p}}\right)\right) \\
& \quad \simeq \mathcal{T}_{i_{p}}^{*}\left(\mathcal{T}_{p+1, q-1}^{*}\left(L\left(i_{q}^{t_{q}}\right)\right) \circ \mathcal{T}_{p+1, q-2}^{*}\left(L\left(i_{q-1}^{t_{q-1}}\right)\right) \circ \cdots \circ L\left(i_{p+1}^{t_{p}}\right)\right)
\end{aligned}
$$

where $\mathcal{T}_{a, b}^{*}:=\mathcal{T}_{i_{a}}^{*} \mathcal{T}_{i_{a+1}}^{*} \cdots \mathcal{T}_{i_{b}}^{*}$ for $1 \leq a \leq b \leq \ell$.

Lemma 5.1. Let $i \in I, w \in \mathrm{W}$, and let $M$ be a simple $R$-module.

(i) Suppose that $M \in \mathscr{C}_{w}$. Then we have

(a) if $s_{i} w<w$ and $\varepsilon_{i}^{*}(M)=0$, then $\mathcal{T}_{i}(M) \in \mathscr{C}_{s_{i} w}$,

(b) if $s_{i} w>w$, then $\mathcal{T}_{i}^{*}(M) \in \mathscr{C}_{s_{i} w}$.

(ii) Suppose that $M \in \mathscr{C}_{*, w}$. Then we have

(a) if $s_{i} w<w$, then $\mathcal{T}_{i}(M) \in \mathscr{C}_{*, s_{i} w}$,

(b) if $s_{i} w>w$ and $\varepsilon_{i}(M)=0$, then $\mathcal{T}_{i}^{*}(M) \in \mathscr{C}_{*, s_{i} w}$.

Proof. Let $w^{\prime}=w^{-1} w_{0} \in \mathrm{W}$ so that $w w^{\prime}=w_{0}$ with $\ell\left(w_{0}\right)=\ell(w)+\ell\left(w^{\prime}\right)$. Let us fix reduced expressions $\underline{w}=s_{i_{1}} s_{i_{2}} \cdots s_{i_{p}}$ and $\underline{w^{\prime}}=s_{j_{1}} s_{j_{2}} \cdots s_{j_{q}}$. We set $\underline{w}^{-}:=s_{i_{2}} \cdots s_{i_{p}}$ and $\underline{w_{0}}:=\underline{w} \underline{w^{\prime}}$. We write the $\preceq \underline{w_{0}}$-decomposition of $M$ as follows:

$$
\mathfrak{d}_{\preceq \underline{w_{0}}}(M)=\left(M_{1}, M_{2}, \ldots, M_{h}\right) .
$$

(i) We assume that $M \in \mathscr{C}_{w}$. Note that $-\operatorname{wt}\left(M_{k}\right) \in \mathbb{Z}_{>0}\left(\Delta_{+} \cap w \Delta_{-}\right)$for any $k$ by Proposition 2.18.

(ia) Suppose that $s_{i} w<w$ and $\varepsilon_{i}^{*}(M)=0$. Without loss of generality, we assume

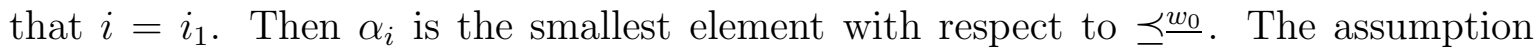
$\varepsilon_{i}^{*}(M)=0$ implies that $\alpha_{i} \prec \frac{w_{0}}{-}-\mathrm{wt}\left(M_{h}\right)$, which implies $\alpha_{i} \prec \underline{w_{0}}-\mathrm{wt}\left(M_{k}\right)$ for $1 \leq$ $k \leq h$. Hence $\alpha_{i} \notin \mathbf{W}^{*}\left(M_{k}\right)$ and $M_{k} \in R_{i}$-mod. By (5.1) and (5.3), $\mathcal{T}_{i}\left(M_{k}\right)$ are 
cuspidal modules with respect to the convex order $\preceq \underline{w}^{-}$satisfying $-\operatorname{wt}\left(\mathcal{T}_{i}\left(M_{k}\right)\right) \succeq \underline{w}^{-}$ $-\operatorname{wt}\left(\mathcal{T}_{i}\left(M_{k+1}\right)\right)$ for $1 \leq k \leq h-1$. It follows from (5.1) and (5.4) that $\mathfrak{d}_{\underline{\underline{w}}^{-}}\left(\mathcal{T}_{i}(M)\right)$ is $\left(\mathcal{T}_{i}\left(M_{1}\right), \mathcal{T}_{i}\left(M_{2}\right), \ldots, \mathcal{T}_{i}\left(M_{h}\right)\right)$. Thus $\mathcal{T}_{i}(M)$ is contained in $\mathscr{C}_{s_{i} w}$ by Proposition 2.18.

(ib) If $s_{i} w>w$, then $\varepsilon_{i}(M)=0$ by Lemma 2.17 and we have $\mathfrak{d}_{\preceq_{i} s_{\underline{w}}}\left(\mathcal{T}_{i}^{*}(M)\right)=$ $\left(\mathcal{T}_{i}^{*}\left(M_{1}\right), \mathcal{T}_{i}^{*}\left(M_{2}\right), \ldots, \mathcal{T}_{i}^{*}\left(M_{h}\right)\right)$ by (5.3) and (5.4). Thus $\mathcal{T}_{i}^{*}(M) \in \mathscr{C}_{s_{i} w}$ by Proposition 2.18 .

(ii) We now assume that $M \in \mathscr{C}_{*, w}$. Note that $-\operatorname{wt}\left(M_{k}\right) \in \mathbb{Z}_{>0}\left(\Delta_{+} \cap w \Delta_{+}\right)$for any $k$ by Proposition 2.18 .

(ii a) Suppose that $s_{i} w<w$. Then $\varepsilon_{i}^{*}(M)=0$ by Lemma 2.17. Without loss of generality, we assume that $i=i_{1}$. Then $-\operatorname{wt}\left(M_{k}\right) \succ \alpha_{i}$ so that $\varepsilon_{i}^{*}\left(M_{k}\right)=0$ for $k=1, \ldots, h$. By $(5.1)$ and $(5.4)$, we have $\mathfrak{d}_{\underline{\underline{w}} \underline{w}^{-}}\left(\mathcal{T}_{i}(M)\right)=\left(\mathcal{T}_{i}\left(M_{1}\right), \mathcal{T}_{i}\left(M_{2}\right), \ldots, \mathcal{T}_{i}\left(M_{h}\right)\right)$. This implies that $\mathcal{T}_{i}(M)$ is contained in $\mathscr{\mathscr { C }}_{*, s_{i} w}$ by Proposition 2.18.

(ii b) Suppose that $s_{i} w>w$ and $\varepsilon_{i}(M)=0$. Let $u=w^{-1} s_{i} w_{0} \in \mathrm{W}$ so that $w u=s_{i} w_{0}$ and $\ell(w u)=\ell(w)+\ell(u)$. Fix a reduced expression $\underline{u}$ of $u$, and let $s_{j}$ be the simple reflection with $s_{i} w_{0}=w_{0} s_{j}$. We set $\preceq^{\prime}:=\preceq \underline{w} \underline{\underline{u}} \underline{s}_{j}$. By the construction, $\alpha_{i}$ is largest among $\Delta_{+}$with respect to the convex order $\preceq^{\prime}$. Let $\left(M_{1}^{\prime}, \ldots, M_{r}^{\prime}\right)$ be the $\preceq^{\prime}$-cuspidal decomposition of $M$. By Proposition 2.18, we know

$$
-\operatorname{wt}\left(M_{k}^{\prime}\right) \succ^{\prime} \beta \quad \text { for } k=1, \ldots, r \text { and } \beta \in \Delta_{+} \cap w \Delta_{-} .
$$

Since $\varepsilon_{i}(M)=0$, we have $-\operatorname{wt}\left(M_{1}^{\prime}\right) \notin \mathbb{Z}_{>0} \alpha_{i}$. Note that $\preceq^{\prime}=\preceq \underline{w} \underline{u}$ as a convex order on $\Delta_{+}$since $\alpha_{i}$ is largest with respect to $\preceq \underline{w} \underline{u}$, and $M_{k}^{\prime} \in \mathscr{C}_{w u}$ for $1 \leq k \leq h$. Thus, applying $\mathcal{T}_{i}{ }^{*}$ to $M$, we have that $\left(\mathcal{T}_{i}{ }^{*}\left(M_{1}^{\prime}\right), \mathcal{T}_{i}{ }^{*}\left(M_{2}^{\prime}\right), \ldots, \mathcal{T}_{i}{ }^{*}\left(M_{r}^{\prime}\right)\right)$ is the $\preceq^{s_{i} \underline{w}} \underline{u}$-cuspidal decomposition of $\mathcal{T}_{i}^{*}(M)$. Moreover, since $-\operatorname{wt}\left(\mathcal{T}_{i}^{*}\left(M_{k}^{\prime}\right)\right)=-s_{i} \operatorname{wt}\left(M_{k}^{\prime}\right)$ is larger than any positive root in $\Delta_{+} \cap s_{i} w \Delta_{-}$with respect to $\preceq^{s_{i} \underline{w} \underline{u}}, \mathcal{T}_{i}^{*}(M)$ is contained in $\mathscr{C}_{*, s_{i} w}$ by Proposition 2.18 .

Remark 5.2. It was proved in [24, Corollary 2.26] that the cuspidal decomposition has compatibility with the Saito crystal reflections $T_{i}$ on simple $R$-modules.

Thanks to Lemma 2.17 and Lemma 5.1, for $w, v \in \mathrm{W}$ with $s_{i} w>w$ and $s_{i} v>v$, the restrictions of the functors

$$
\left.\mathcal{T}_{i}\right|_{\mathscr{C}_{s_{i} w, s_{i} v}}: \mathscr{C}_{s_{i} w, s_{i} v} \longrightarrow \mathscr{C}_{w, v},\left.\quad \mathcal{T}_{i}^{*}\right|_{\mathscr{C}_{w, v}}: \mathscr{C}_{w, v} \longrightarrow \mathscr{C}_{s_{i} w, s_{i} v}
$$

are well-defined and quasi-inverse to each other by (5.1). Thus, we have the following.

Theorem 5.3. Let $w, v \in \mathrm{W}$ with $v \leq w, s_{i} w>w$ and $s_{i} v>v$. Then the restrictions of the functors

$$
\left.\mathcal{T}_{i}\right|_{\mathscr{C}_{s_{i} w, s_{i} v}}: \mathscr{C}_{s_{i} w, s_{i} v} \stackrel{\sim}{\longrightarrow} \mathscr{C}_{w, v},\left.\quad \mathcal{T}_{i}^{*}\right|_{\mathscr{C}_{w, v}}: \mathscr{C}_{w, v} \stackrel{\sim}{\longrightarrow} \mathscr{C}_{s_{i} w, s_{i} v}
$$


give equivalences of the categories.

Corollary 5.4. Let $w, u, v \in \mathrm{W}$ such that $w=v u$ and $\ell(w)=\ell(v)+\ell(u)$. Then there is an equivalence of the categories between $\mathscr{C}_{w, v}$ and $\mathscr{C}_{u}$.

Let $w, v \in \mathrm{W}$ with $w>v$. For a reduced expression $\underline{w}$ of $w$, we define

$$
J_{\underline{w}, v}:=\left\{k \in\{1,2, \ldots, \ell(w)\} \mid v_{\leq k}=v_{\leq k-1}\right\},
$$

where $v_{\leq k}$ is defined in (4.3). Note that $\left|J_{\underline{w}, v}\right|=\ell(w)-\ell(v)$.

Suppose that $w, v, u \in \mathrm{W}$ such that $w=v u$ and $\ell(w)=\ell(v)+\ell(u)$. We fix reduced expressions $\underline{v}=s_{i_{1}} \cdots s_{i_{p}}$ and $\underline{u}=s_{i_{p+1}} \cdots s_{i_{q}}$ of $v$ and $u$ respectively, and set $\underline{w}=\underline{v} \underline{u}$. Note that (for the notation see (5.5))

$$
J_{\underline{w}, v}=\{p+1, p+2, \ldots, q\} .
$$

We define

Then (5.2) gives

$$
\mathbb{W}:=\mathcal{T}_{i_{1}}^{*} \cdots \mathcal{T}_{i_{p}}^{*}: \mathscr{C}_{u} \stackrel{\sim}{\longrightarrow} \mathscr{C}_{w, v}
$$

$$
\begin{aligned}
\mathbb{W}\left(\mathrm{M}\left(s_{i_{p+1}} \cdots s_{i_{p+k}} \Lambda_{i_{p+k}}, \Lambda_{i_{p+k}}\right)\right) & \simeq \mathrm{M}\left(v s_{i_{p+1}} \cdots s_{i_{p+k}} \Lambda_{i_{p+k}}, v \Lambda_{i_{p+k}}\right) \\
& =\mathrm{M}\left(w_{\leq p+k} \Lambda_{i_{p+k}}, v_{\leq p+k} \Lambda_{i_{p+k}}\right)
\end{aligned}
$$

for $k=1, \ldots, q$. It was proved in [7] that the category $\mathscr{C}_{u}$ and the determinantial modules

$$
\left\{\mathrm{M}\left(s_{i_{p+1}} \cdots s_{i_{p+k}} \Lambda_{i_{p+k}}, \Lambda_{i_{p+k}} \mid 1 \leq k \leq q\right\}\right.
$$

give a monoidal categorification of $A_{q}\left(\mathfrak{n}\left(u^{-1}\right)\right)_{\mathbb{Z}\left[q, q^{-1}\right]}$.

Conjecture 5.5. Let $w, v, u \in \mathrm{W}$ such that $w=v u$ and $\ell(w)=\ell(v)+\ell(u)$. Then, the category $\mathscr{C}_{w, v}$ with the determinantial modules $\mathrm{M}\left(w_{\leq k} \Lambda_{i_{k}}, v_{\leq k} \Lambda_{i_{k}}\right)$ for $k \in J_{\underline{w}, v}$ give the monoidal categorification of $A_{w, v}=K_{0}\left(\mathscr{C}_{w, v}\right)$ induced from that of $A_{q}\left(\mathfrak{n}\left(u^{-1}\right)\right)_{\mathbb{Z}\left[q, q^{-1}\right]}$ via the functor $\mathbb{W}$.

Remark 5.6. It was shown in [11, Section 4] that the exact functor $\mathcal{T}_{i}$ is compatible with the convolution product in the case of only PBW modules. If $\mathcal{T}_{i}$ and $\mathcal{T}_{i}{ }^{*}$ are monoidal functors, then it implies that Conjecture 5.5 is true. Indeed, if $\mathbb{W}$ is a monoidal functor, then we have a $\mathbb{Z}\left[q, q^{-1}\right]$-algebra isomorphism from $A_{q}\left(\mathfrak{n}\left(u^{-1}\right)\right)_{\mathbb{Z}\left[q, q^{-1}\right]}$ to $A_{w, v}$. Recall that

$$
\left(\left\{q^{-\left(d_{k}, d_{k}\right) / 4}\left[M_{k}\right]\right\}_{1 \leq k \leq q}, L, \widetilde{B}\right)
$$

gives an initial seed of the quantum cluster algebra $\mathbb{Q}\left(q^{1 / 2}\right) \otimes_{\mathbb{Z}\left[q, q^{-1}\right]} A_{q}\left(\mathfrak{n}\left(u^{-1}\right)\right)_{\mathbb{Z}\left[q, q^{-1}\right]}$ where $M_{k}=\mathrm{M}\left(s_{i_{p+1}} \cdots s_{i_{p+k}} \Lambda_{i_{p+k}}, \Lambda_{i_{p+k}}\right), \quad d_{k}=\operatorname{wt}\left(M_{k}\right), \quad L=-\left(\Lambda\left(M_{i}, M_{j}\right)\right)_{1 \leq i, j \leq q}$ and $\tilde{B}$ is the skew-symmetric matrix given in [7, Definition 11.1.1]. It follows that 
$\mathbb{Q}\left(q^{1 / 2}\right) \otimes_{\mathbb{Z}\left[q, q^{-1}\right]} A_{w, v}$ is equipped with a quantum cluster algebra structure with an initial seed

$$
\left(\left\{q^{-\left(d_{k}, d_{k}\right) / 4}\left[\mathbb{W}\left(M_{k}\right)\right]\right\}_{1 \leq k \leq q}, L, \widetilde{B}\right) .
$$

Moreover, the pair $\left(\left\{M_{k}\right\}_{1 \leq k \leq q}, \widetilde{B}\right)$ is admissible in the sense [7, Definition 7.1.1]. The key condition is that for each mutable index $k$, there exists a self-dual simple object $M_{k}^{\prime}$ of $\mathscr{C}_{u}$ such that there is an exact sequence in $\mathscr{C}_{u}$

$$
0 \rightarrow q \bigodot_{b_{i k}>0} M_{i}^{\odot b_{i k}} \rightarrow q^{\widetilde{\Lambda}\left(M_{k}, M_{k}^{\prime}\right)} M_{k} \circ M_{k}^{\prime} \rightarrow \bigodot_{b_{i k}<0} M_{i}^{\odot\left(-b_{i k}\right)} \rightarrow 0,
$$

where $\odot$ denotes a grading shift of the usual convolution product which depends only on $L$. By applying $\mathbb{W}$, we get an exact sequence in $\mathscr{C}_{w, v}$

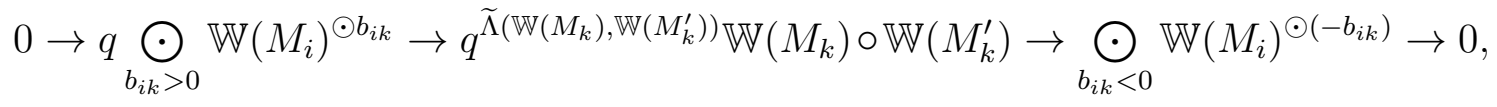

since we have $\Lambda(M, N)=\Lambda(\mathbb{W}(M), \mathbb{W}(N))$ for any real simple modules $M, N \in \mathscr{C}_{u}$. In turn, the pair in $\mathscr{C}_{w, v}$

$$
\left(\left\{\mathrm{M}\left(w_{\leq p+k} \Lambda_{i_{p+k}}, v_{\leq p+k} \Lambda_{i_{p+k}}\right)\right\}_{1 \leq k \leq q}, \widetilde{B}\right)
$$

is admissible. Then, by [7, Theorem 7.1.3], we conclude that $\mathscr{C}_{w, v}$ is a monoidal categorification of the quantum cluster algebra $A_{w, v}$.

Remark 5.7. Let us recall the notations in Remark 2.19. Under the assumption on $w$ and $v$ in Conjecture 5.5, the unipotent group $N_{v}^{w}$ of $N$ corresponding to the positive roots in $\Delta_{+} \cap w \Delta_{-} \cap v \Delta_{+}$is a natural fundamental domain for the action $N^{\prime}(w) \times N(v)$ on $N$. As explained in [17, Section 5], the coordinate ring $\mathbb{C}\left[N_{v}^{w}\right]$ has a cluster structure which is isomorphic to that of $\mathbb{C}[N(u)]$. Thus, Conjecture 5.5 tells that the category $\mathscr{C}_{w, v}$ is a monoidal categorification of a quantization of the cluster algebra $\mathbb{C}\left[N_{v}^{w}\right]$ in terms of quiver Hecke algebras.

\section{REFERENCES}

[1] J. Brundan and A. Kleshchev, Blocks of cyclotomic Hecke algebras and Khovanov-Lauda algebras, Invent. Math. 178 (2009), 451-484.

[2] J. Hong and S.-J. Kang, Introduction to Quantum Groups and Crystal Bases, Graduate Studies in Mathematics, 42. American Mathematical Society, Providence, RI, 2002.

[3] C. Geiß, B. Leclerc and J. Schröer, Cluster structures on quantum coordinate rings, Selecta Math. (N.S.) 19 (2013), no. 2, 337-397.

[4] S.-J. Kang and M. Kashiwara, Categorification of Highest Weight Modules via Khovanov-LaudaRouquier Algebras, Invent. Math. 190 (2012), no. 3, 699-742. 
[5] S.-J. Kang, M. Kashiwara and M. Kim, Symmetric quiver Hecke algebras and R-matrices of quantum affine algebras, Invent. Math. 211 (2018), no. 2, 591-685.

[6] S.-J. Kang, M. Kashiwara, M. Kim and S.-j. Oh, Simplicity of heads and socles of tensor products, Compos. Math. 151 (2015), no. 2, 377-396.

[7] _ Monoidal categorification of cluster algebras, J. Amer. Math. Soc. 31 (2018), no. 2, $349-426$.

[8] M. Kashiwara, On crystal bases of the q-analogue of universal enveloping algebras, Duke Math. J. 63 (1991) 465-516.

[9] _ Global crystal bases of quantum groups, Duke Math. J. 69 (1993), no. 2, 455-485.

[10] M. Kashiwara and E. Park, Affinizations and R-matrices for quiver Hecke algebras, arXiv:1505.03241; to appear in Journal of the European Mathematical Society.

[11] S. Kato, Poincaré-Birkhoff-Witt bases and Khovanov-Lauda-Rouquier algebras, Duke Math. J. 163 (2014), no. 3, 619-663.

[12] _ On the monoidality of Saito reflection functors, arXiv:1711.09085.

[13] M. Khovanov and A. Lauda, A diagrammatic approach to categorification of quantum groups I, Represent. Theory 13 (2009), 309-347.

[14] _ A diagrammatic approach to categorification of quantum groups II, Trans. Amer. Math. Soc. 363 (2011), 2685-2700.

[15] Y. Kimura, Quantum unipotent subgroup and dual canonical basis, Kyoto J. Math. 52 (2012), no. $2,277-331$.

[16] A. Lauda and M. Vazirani, Crystals from categorified quantum groups, Adv. Math. 228 (2011), no. $2,803-861$.

[17] B. Leclerc, Cluster structures on strata of flag varieties, Adv. Math. 300 (2016), 190-228.

[18] T. H. Lenagan and M. T. Yakimov, Prime factors of quantum Schubert cell algebras and clusters for quantum Richardson varieties, arXiv:1503.06297; to appear in J. reine angew. Math. (DOI: https://doi.org/10.1515/crelle-2016-0046)

[19] P. J. McNamara, Monoidality of Kato's Reflection Functors, arXiv:1712.00173.

[20] P. J. McNamara and P. Tingley, Face functors for KLR algebras, Represent. Theory 21 (2017), $106-131$.

[21] R. Rouquier, 2-Kac-Moody algebras, arXiv:0812.5023v1.

[22] _ Quiver Hecke algebras and 2-Lie algebras, Algebra Colloq. 19 (2012), no. 2, 359-410.

[23] Y. Saito, $P B W$ basis of quantized universal enveloping algebras, Publ. Res. Inst. Math. Sci. 30 (1994), no. 2, 209-232.

[24] P. Tingley and B. Webster, Mirković-Vilonen polytopes and Khovanov-Lauda-Roquuier algebras, Compos. Math. 152 (2016), no. 8, 1648-1696.

[25] M. Varagnolo and E. Vasserot, Canonical bases and KLR algebras, J. Reine Angew. Math. 659 (2011), 67-100. 
(Masaki Kashiwara) Research Institute for Mathematical Sciences, Kyoto University, Kyoto 606-8502, Japan \& Korea Institute for Advanced Study, Seoul 02455, Korea

E-mail address, Masaki Kashiwara: masaki@kurims.kyoto-u.ac.jp

(Myungho Kim) Department of Mathematics, Kyung Hee University, Seoul 02447, KOREA

E-mail address, Myungho Kim: mkim@khu.ac.kr

(Se-jin Oh) Department of Mathematics, Ewha Womans University, Seoul 120-750, KOREA

E-mail address, Se-jin Oh: sejin092@gmail.com

(Euiyong Park) Department of Mathematics, University of Seoul, Seoul 02504, Korea

E-mail address, Euiyong Park: epark@uos.ac.kr 